\title{
Two scales hydrodynamic limit for a model of malignant tumor cells
}

\section{Limite hydrodynamique à deux échelles pour un modèle de cellules tumorales malignes}

\author{
Anna De Masi ${ }^{a, *}$, Stephan Luckhaus ${ }^{b}$, Errico Presutti $^{\mathrm{c}}$ \\ a Dipartimento di Matematica Pura ed Applicata, Università di L'Aquila, Via Vetoio (Coppito) 67100 L'Aquila, Italy \\ ${ }^{\mathrm{b}}$ University of Leipzig, Augustus Platz, 10-11, 04109 Leipzig, Germany \\ c Dipartimento di Matematica, Università di Roma "Tor Vergata", 00133 Roma, Italy
}

Received 15 February 2005; received in revised form 24 February 2006; accepted 22 March 2006

Available online 25 September 2006

\begin{abstract}
We consider a model introduced in [S. Luckhaus, L. Triolo, The continuum reaction-diffusion limit of a stochastic cellular growth model, Rend. Acc. Lincei (S.9) 15 (2004) 215-223] with two species ( $\eta$ and $\xi$ ) of particles, representing respectively malignant and normal cells. The basic motions of the $\eta$ particles are independent random walks, scaled diffusively. The $\xi$ particles move on a slower time scale and obey an exclusion rule among themselves and with the $\eta$ particles. The competition between the two species is ruled by a coupled birth and death process. We prove convergence in the hydrodynamic limit to a system of two reaction-diffusion equations with measure valued initial data.
\end{abstract}

๑) 2006 Elsevier Masson SAS. All rights reserved.

\section{Résumé}

Nous considérons un modèle introduit dans [S. Luckhaus, L. Triolo, The continuum reaction-diffusion limit of a stochastic cellular growth model, Rend. Acc. Lincei (S.9) 15 (2004) 215-223] avec deux espèces de particules ( $\eta$ et $\xi$ ) représentant respectivement les cellules malignes et saines. Les mouvements de base des cellules $\eta$ sont des marches aléatoires indépendantes, sur une échelle diffusive. Les particules $\xi$ se déplacent sur une échelle plus lente et obéissent à une règle d'exclusion entre elles et avec les particules $\eta$. La compétition entre les deux espèces est définie par un processus couplé de naissances et morts. Nous prouvons la convergence au sens de la limite hydrodynamique vers un système de deux équations de réaction-diffusion avec données initiales à valeurs mesures.

(c) 2006 Elsevier Masson SAS. All rights reserved.

MSC: $60 \mathrm{~K} 35 ; 82 \mathrm{C} 22$

Keywords: Interacting particle systems; Hydrodynamical limit

\footnotetext{
* Corresponding author.

E-mail addresses: demasi@univaq.it (A. De Masi), luckhaus@mis.mpg.de (S. Luckhaus), presutti@mat.uniroma2.it (E. Presutti).
} 


\section{Introduction}

We are investigating a model of competition between malignant and normal cells for tumor growth that was introduced in [10]. The two main qualitative features which distinguish the behavior of normal and tumoral cells according to [10] are: a lateral contact inhibition for normal cells which is instead absent for malignant cells and that malignant cells have a significantly higher mobility than normal cells. Lateral contact inhibition means roughly that normal cells stop growing when coming in contact with another cell and cannot move on top of another cell, so they stay in a layer (as indicated by the vitro cultivation). Malignant cells on the other hand divide and move without restrictions. Competition arises by supposing that the death-birth rates of a cell depend on the total (local) cell density.

To model such features we consider as in [10] a particles system on $\mathbb{Z}^{d}, d=2$, in the biological case, with two species of particles. Normal cells are represented by an exclusion process $\xi(x), x \in \mathbb{Z}^{d}, \xi(x)=0,1$. Malignant cells instead have no exclusion and they are described by variables $\eta(x)$ taking values in $\mathbb{N}$.

The evolution is described by three processes, the motion of the $\eta$ particles, the motion of the $\xi$ particles and a birth-death process involving $\xi$ and $\eta$ particles. Motivated by the above considerations we suppose that there is a sharp time-scale separation between the three processes and this will be the key assumption which in the end will allow us to derive a continuum description of the model in terms of a system of reaction-diffusion equations. The fastest process is that of the $\eta$ particles which move as independent symmetric random walks. At a much slower rate but still very fast in macroscopic time units move the $\xi$ particles which are also symmetric random walks, but with the rule that jumps on sites occupied either by $\xi$ or $\eta$ particles or both are suppressed (we will refer to this for brevity as a coupled exclusion). Notice that including the exclusion also against $\eta$ particles introduces a first interaction between the two species which in fact will be the most dangerous one, as it is as frequent as the motion of the $\xi$ particles and thus very strong in macroscopic time units. Finally, the slowest time scale, the macroscopic scale, is the one where births and deaths occur, modelling the competition between the two species.

There are many works in the literature on the derivation of reaction-diffusion equations. The main assumption in all these papers is that interactions occur on the macroscopic time scale while the particles move on a much faster scale either as independent or as stirring. These two processes are very well studied and in particular their invariant measures are known being products of Poisson and Bernoulli measures. Then using either "entropy methods" or "correlation functions techniques" it is relatively easy to study the continuum limit, deriving reaction diffusion equations. To exploit such results the coupled exclusion described above was replaced in [10] by a stirring process. Under such an assumption, the invariant measures for the process without births and deaths are products of Poisson and Bernoulli measures and the "classical" techniques can be adapted to derive the continuum limit, as proved in [10].

Here we deal with the original model, with coupled exclusion, where the global invariant measures are not known, even if births and deaths are neglected. However, at least heuristically, the fact that on the time scale of the exclusion process the random walks of the $\eta$ particles have already averaged out their positions, should give rise to an effective process which is again the stirring with slowly varying coefficient process considered in [10]. But the usual methods of deriving hydrodynamic limits do not seem to apply here: the lack of knowledge of the invariant measures for the process without births and deaths seems to preclude the use of entropy methods. We refer to [9] for a general survey on entropy methods in hydrodynamic limits and to [11,12] for specific applications to reaction-diffusion equations. On the other hand, the presence in the reaction terms of transcendental, non polynomial functions, makes awkward an analysis of the BBGKY hierarchy of the type proposed in $[4,2,1]$. However even with polynomial rates the extension of the correlation functions method to the present case is far from trivial.

So here we introduce a new method. The main point is that for our system, the energy estimate which holds for the deterministic limit, can also be obtained for mesoscopic empirical averages of the particles occupation number variables, obtaining $H_{1}^{2}$ a-priori bounds which allow to derive a substitute for the so called "two block estimate" of [8].

We also get bounds for the $L^{2}$ distance of our mesoscopic field variables from the deterministic solutions, derived by an explicit computation of the generator applied to the difference squared. To control the "most dangerous terms" we use homogenization techniques which play the role of the "one block estimates" in hydrodynamic limits, see again [8].

The different scales reflect also in the initial data. We suppose regularity of the $\eta$-particles initial distribution on the macroscopic scale, while the $\xi$-particles distribution is only smooth on a smaller scale, related to the slower time scaling of their time evolution. This involves yet another homogenization process which leads to a limit coupled system of reaction-diffusion equations with measure valued initial data for the normal cells. 
In Section 2 we define the particles process, in Section 3 a discrete deterministic system of reaction-diffusion equations and in Section 4 mesoscopic variables. In Section 5 we discuss the choice of the initial state and in Section 6 we state our main results. In Section 7 we give a brief sketch of the proofs. In Section 8 we prove a priori bounds on exponential moments of the occupation variables and regularity in space of the mesoscopic fields. In Section 9 we prove regularity in time, while in Sections 10 and 11 we prove local ergodic theorems (one block estimates) for the $\eta$ and, respectively, $\xi$ particles. We have shifted to some appendices the more computational parts of the proofs.

\section{The particles model}

For simplicity we will consider periodic boundary conditions and thus the macroscopic system will live in a unit torus $\Omega$ of $\mathbb{R}^{d}$. To introduce our particles model we then discretize $\Omega$ by intersecting it with the lattice $\epsilon \mathbb{Z}^{d}, \epsilon^{-1} \in \mathbb{N}$, and denote by $\Omega_{\epsilon}$ its image in stretched coordinates:

$$
\Omega_{\epsilon}=\left\{x=\left(x_{1}, \ldots, x_{d}\right) \in \mathbb{Z}^{d}: 1 \leqslant x_{i} \leqslant \epsilon^{-1}, 1 \leqslant i \leqslant d\right\} .
$$

As a rule we will use $x, y, z, \ldots$ for lattice sites and $r, r^{\prime}, \ldots$ for points in $\mathbb{R}^{d}$.

Particle configurations are non negative integer valued functions on $\Omega_{\epsilon}$ extended periodically to the whole $\mathbb{Z}^{d}$; in particular we consider configurations $\eta: \mathbb{Z}^{d} \rightarrow \mathbb{N}$ and $\xi: \mathbb{Z}^{d} \rightarrow\{0,1\} . \eta(x)$ and $\xi(x), x \in \mathbb{Z}^{d}$, are thus the number of $\eta$ and $\xi$ particles at any site $y$ in $\Omega_{\epsilon}$ equal to $x$ modulo $\Omega_{\epsilon} . \eta$ and $\xi$ are interpreted as malignant and respectively normal cells.

The evolution is described by a Markov process whose generator $L$, defined on all functions of $(\eta, \xi)$ and whose dependence on $\epsilon$ is not made explicit, has the expression

$$
\begin{aligned}
& L=L^{(\eta)}+L^{(\xi)}, \\
& L^{(\eta)}=\epsilon^{-2} L^{(\eta, 0)}+L^{(\eta,+)}+L^{(\eta,-)}, \\
& L^{(\xi)}=\epsilon^{-2 a} L^{(\xi, 0)}+L^{(\xi,+)}+L^{(\xi,-)}, \quad a \in(0,1),
\end{aligned}
$$

with

$$
L^{(\eta, 0)} f(\eta, \xi)=\sum_{x \in \Omega_{\epsilon}} \sum_{e:|e|=1} \eta(x)\left[f\left(\eta^{x, x+e}, \xi\right)-f(\eta, \xi)\right]
$$

where, denoting by $1_{x}$ the configuration with only one particle at $x, \eta^{x, x+e}=\eta+1_{x+e}-1_{x}$ (same notation is used for $\xi$ configurations).

$$
L^{(\xi, 0)} f(\eta, \xi)=\sum_{x \in \Omega_{\epsilon}} \sum_{e:|e|=1} \xi(x)[1-\xi(x+e)] \mathbf{1}_{\eta(x+e)=0}\left[f\left(\eta, \xi^{x, x+e}\right)-f(\eta, \xi)\right] .
$$

Denoting below by $\kappa, \kappa^{\prime}$ and $\kappa_{i}$ positive coefficients, the latter non decreasing functions of $i$ bounded by $\kappa_{i} \leqslant c \mathrm{e}^{b i}$, $b$ and $c$ positive constants, and writing $\eta^{x, \pm}=\eta \pm 1_{x}, \xi^{x, \pm}=\xi \pm 1_{x}$,

$$
\begin{aligned}
& L^{(\eta,+)} f(\eta, \xi)=\sum_{x \in \Omega_{\epsilon}} \kappa \eta(x)\left[f\left(\eta^{x,+}, \xi\right)-f(\eta, \xi)\right], \\
& L^{(\eta,-)} f(\eta, \xi)=\sum_{x \in \Omega_{\epsilon}} \eta(x)\left(\kappa_{\eta(x)}(1-\xi(x))+\kappa_{\eta}(x)+1 \xi(x)\right)\left[f\left(\eta^{x,-}, \xi\right)-f(\eta, \xi)\right], \\
& L^{(\xi,+)} f(\eta, \xi)=\sum_{x \in \Omega_{\epsilon}} \frac{\kappa^{\prime}}{2 d} \sum_{e:|e|=1} \xi(x)[1-\xi(x+e)] \mathbf{1}_{\eta(x+e)=0}\left[f\left(\eta, \xi^{x+e,+}\right)-f(\eta, \xi)\right], \\
& L^{(\xi,-)} f(\eta, \xi)=\sum_{x \in \Omega_{\epsilon}} \xi(x) \kappa_{\eta(x)+1}\left[f\left(\eta, \xi^{x,-}\right)-f(\eta, \xi)\right] .
\end{aligned}
$$

The indices $0,+,-$ above refer respectively to displacements, births and deaths of particles, whose species is then indicated by $\eta$ and $\xi$. Notice that, as $\epsilon \rightarrow 0$, the scaling factors $\epsilon^{-2}$ and $\epsilon^{-2 a}$ make displacements occur on a much faster scale than births and deaths with the $\eta$ particles moving much faster than the $\xi$ particles, because $a \in(0,1)$. 
$L^{(\eta, 0)}$ is the generator of independent symmetric random walks on $\Omega_{\epsilon}$. Due to the presence of the characteristic function $\mathbf{1}_{\eta(x+e)=0}, L^{(\xi, 0)}$ differs from the stirring generator

$$
L^{(\xi, \mathrm{st})} f(\eta, \xi)=\sum_{x \in \Omega_{\epsilon}} \sum_{e:|e|=1} \xi(x)[1-\xi(x+e)]\left[f\left(\eta, \xi^{x, x+e}\right)-f(\eta, \xi)\right]
$$

and the motion of the $\xi$ particles is coupled under $L^{(\xi, 0)}$ to the evolution of the $\eta$ particles, this is the coupled exclusion mentioned in the introduction.

\section{Discrete reaction-diffusion equations}

The limit reaction diffusion equations that we will derive, can be approximated on the lattice $\Omega_{\epsilon}$, by the two equations

$$
\frac{\mathrm{d} U}{\mathrm{~d} t}=F(U, V), \quad \frac{\mathrm{d} V}{\mathrm{~d} t}=G(U, V)
$$

$(U(t), V(t))=\left\{(U(x, t), V(x, t)), x \in \Omega_{\epsilon}, t \geqslant 0\right\}, F=F(U, V)$ and $G=G(U, V)$ being given by

$$
\begin{aligned}
& F(U, V)=\epsilon^{-2} \Delta U+\kappa U-F_{1}^{-}(U)-F_{2}^{-}(U) V, \\
& G(U, V)=\mathrm{e}^{-U} \epsilon^{-2 a} \Delta V+\kappa^{\prime} V(1-V) \mathrm{e}^{-U}-V G^{-}(U)
\end{aligned}
$$

with $\Delta$ the discrete Laplacian on $\mathbb{Z}^{d}, \Delta f(x)=\sum_{i=1}^{d}\left[f\left(x+e_{i}\right)+f\left(x-e_{i}\right)-2 f(x)\right], e_{i}$ the unit vector along the positive $i$ th coordinate direction,

$$
F_{1}^{-}(U)=\mathrm{e}^{-U} \sum_{i \geqslant 0} \frac{U^{i}}{i !} i \kappa_{i}, \quad F_{2}^{-}(U)=\mathrm{e}^{-U} \sum_{i \geqslant 0} \frac{U^{i}}{i !} i\left[\kappa_{i+1}-\kappa_{i}\right], \quad G^{-}(U)=\mathrm{e}^{-U} \sum_{i \geqslant 0} \frac{U^{i}}{i !} \kappa_{i+1} .
$$

In Section 6 we will rewrite (3.1) in un-stretched coordinates, $x \rightarrow \epsilon x, \Omega_{\epsilon} \rightarrow \Omega \cap \epsilon \mathbb{Z}^{d}$ and $\epsilon^{-2} \Delta \rightarrow \Delta$ and its limit behavior as $\epsilon \rightarrow 0$ will become more transparent. (3.2) and (3.3) are obtained by averaging the rates of change of $\eta(\cdot)$ and $\xi(\cdot)$ using products of Poisson measures for the $\eta$ 's and product measures for the $\xi$ 's with parameters depending on $U$ and $V$.

Our aim is to prove closeness between (3.1) and the particles process of the previous section. Of course there is no chance that $\eta_{t}$ and $\xi_{t}$ are pointwise close to $U(t)$ and $V(t)$ as the former are truly random variables. However by taking empirical averages we can dampen the statistical fluctuations. We will thus introduce suitable functions $(u(x, \eta), v(x, \xi)), x \in \Omega_{\epsilon}$, defined as convolutions with suitable kernels of the original $\eta$ and $\xi$ variables, and compare their evolution with the orbits $(U(t), V(t))$ of (3.1). An important step in this direction will be a comparison between the generator $L$ and the generator $D$ associated to (3.1), $D$ being the transport operator with domain the space of all functions $\psi(U, V)$ which are differentiable in all $U(x)$ and $V(x)$, and which is defined as

$$
D=F \frac{\partial}{\partial U}+G \frac{\partial}{\partial V} \equiv \sum_{x \in \Omega_{\epsilon}} F(x) \frac{\partial}{\partial U(x)}+G(x) \frac{\partial}{\partial V(x)},
$$

where $F(x)$ and $G(x)$ are $F(U, V)$ and $G(U, V)$ computed at $x$. Thus if $h=h(U, V), D h(U, V)$ is the $t$-derivative of $h(U(t), V(t))$ at $t=0$, where $U(t), V(t)$ is the solution of (3.1) starting at $U, V . D^{(\eta, 0)}, D^{(\xi, 0)}$ and so forth are defined by decomposing $D$ in the same way we did for $L$ in the previous section and we will compare $D^{(\eta, 0)}$ with $L^{(\eta, 0)}$ and so on.

\section{Mesoscopic variables}

We denote by $\pi_{t}$ the semigroup

$$
\pi_{t}=\mathrm{e}^{t \Delta}
$$

with $\Delta$ the discrete Laplacian on $\Omega_{\epsilon}$, so that the kernel $\pi_{t}(x, y)$ is the probability that a simple random walk which jumps with intensity $2 d$ with equal probability on its n.n. sites, reaches $y$ at time $t$ having started from $x$ at time 0 . 
With $\alpha<1$ and $\beta<a$ positive parameters whose value will be specified at the end of this section, we shorthand

$$
u(\eta)=p_{\alpha} * \eta, \quad v(\xi)=q_{\beta} * \xi, \quad p_{\alpha}=\pi_{\epsilon^{-2+2 \alpha}}, \quad q_{\beta}=\pi_{\epsilon^{-2 a+2 \beta}},
$$

where if $p=p(x, y)$ is a kernel on $\Omega_{\epsilon}$ and $f=f(x)$ a function on $\Omega_{\epsilon}$ we denote by $p * f$ the function on $\Omega_{\epsilon}$ defined by $p * f(x)=\sum_{y \in \Omega_{\epsilon}} p(x, y) f(y)$. We will denote by $u(x, \eta)$ and $v(x, \xi)$ the values at $x$ of the functions $u$ and $v$ defined in (4.2) and use throughout the paper the notation:

$$
\langle f, g\rangle=\epsilon^{d} \sum_{x \in \Omega_{\epsilon}} f(x) g(x), \quad\|f\|^{2}=\langle f, f\rangle .
$$

The key for proving closeness between $\left(u\left(\eta_{t}\right), v\left(\xi_{t}\right)\right)$ and $(U(t), V(t))$ will be a proof that $L$ acts on functions of $(u, v)$ approximately as $D$ acts on functions of $(U, V)(D$ as in (3.4)). We will make the statement quantitative, by studying the quantities

$$
(L+D)\|u-U\|^{2}, \quad(L+D)\|v-V\|^{2}
$$

along the trajectories of the Markov process and the solutions of (3.1). Thus if $(u, v)$ are close to $(U, V)$ and $L$ to $D$, then the quantities in (4.3) will be small. The converse is (to some extent) also true, as it follows from the martingale theory. Indeed the expressions in (4.3) are the two compensators in the martingale relations

$$
\begin{aligned}
& \left\|u\left(\eta_{t}\right)-U(t)\right\|^{2}-\left\|u\left(\eta_{0}\right)-U(0)\right\|^{2}=\int_{0}^{t}(L+D)\left\|u\left(\eta_{s}\right)-U(s)\right\|^{2} \mathrm{~d} s+M_{1}(t), \\
& \left\|v\left(\xi_{t}\right)-V(t)\right\|^{2}-\left\|v\left(\xi_{0}\right)-V(0)\right\|^{2}=\int_{0}^{t}(L+D)\left\|v\left(\xi_{s}\right)-V(s)\right\|^{2} \mathrm{~d} s+M_{2}(t),
\end{aligned}
$$

where $(U(s), V(s))$ solves (3.1) and $M_{1}(t), M_{2}(t)$ are martingales.

We will prove bounds for the two integrals on the right-hand side of (4.4) and (4.5) in terms of $\int_{0}^{t}\left(\| u\left(\eta_{s}\right)-\right.$ $\left.U(s)\left\|^{2}+\right\| v\left(\xi_{s}\right)-V(s) \|^{2}\right) \mathrm{d} s$. We will also show that with probability going to 1 the martingales vanish in the limit as $\epsilon \rightarrow 0$. All that will allow to reach an integral inequality in closed form for $\left\|u\left(\eta_{s}\right)-U(s)\right\|^{2}+\left\|v\left(\xi_{s}\right)-V(s)\right\|^{2}$ and to prove that the solution vanishes in the limit $\epsilon \rightarrow 0$.

Choice of $\boldsymbol{\alpha}$ and $\boldsymbol{\beta}$, assumptions on $\boldsymbol{a}$. Recall that $\alpha<1$ and $\beta<a<1$. Let

$$
\begin{aligned}
& 0<a<\min \left\{\frac{d}{d+2}, \frac{d}{10}, \frac{d^{2}}{8(d+2)}\right\}, \\
& \max \left\{2 a, \frac{8 a}{d}\right\}<\alpha<\frac{d}{d+2}
\end{aligned}
$$

and

$$
\frac{5 a}{17}<\beta<\min \left\{\frac{a d}{d+2}, \frac{\alpha}{2}, \frac{1}{2 d}(1-\alpha)\right\} .
$$

With such a choice:

- $\alpha<d /(d+2)$ (see the proof of Theorem 8.2), $\alpha>2 a$ and $\alpha>8 a / d$ (see Appendix D).

- $2 a<1,2 a<(1-\alpha) d, \beta<a d /(d+2)$ (see the proof of Theorem 8.3).

- $\beta>5 a / 17$ and $2 \beta<\min (\alpha,(1-\alpha) / d$ ) (see the proof of Theorem 11.1).

Properties of $\boldsymbol{p}_{\boldsymbol{\alpha}}$ and $\boldsymbol{q}_{\boldsymbol{\beta}}$. We list below some properties of $p_{\alpha}$ and $q_{\beta}$ which follow from the local central limit Theorem and will be often used in the sequel: There is a constant $c>0$ so that

$$
\begin{aligned}
& \sum_{z} \epsilon^{-2}\left|\nabla p_{\alpha}(0, z)\right|^{2} \leqslant c \epsilon^{-2+2(1-\alpha)+d(1-\alpha)} \sum_{z} p_{\alpha}(0, z)^{2} \leqslant c \epsilon^{d(1-\alpha)}, \\
& \sum_{z}\left|\nabla q_{\beta}(0, z)\right|^{2} \leqslant c \epsilon^{2(a-\beta)+d(a-\beta)},
\end{aligned}
$$




$$
\begin{aligned}
& \sum_{z} q_{\beta}(0, z)^{2} \leqslant c \epsilon^{d(a-\beta)}, \\
& \left|\sum_{z} z e \cdot \nabla q_{\beta}(0, z)\right| \leqslant c .
\end{aligned}
$$

\section{Choice of the initial state}

In this section we choose $\mu^{\epsilon}$, the initial law of the process. It is now convenient to underline dependence on $\epsilon$ and we do that by adding a superscript $\epsilon$ when needed. The picture we have in mind is that the $\eta$ and the $\xi$ particles move freely (independently of each other and with no births and deaths) till a finite time $t_{0}$ when the interaction is suddenly switched on. The evolution after $t_{0}$ is then ruled by the generator $L$. We will count times from when the interaction starts thus setting this time equal to 0 .

We fix a ( $\epsilon$ dependent) initial configuration at time $-t_{0},\left(\eta_{-t_{0}}^{\epsilon}, \xi_{-t_{0}}^{\epsilon}\right)$. Due to the general class of processes that we are considering, it is convenient to assume that

$$
\sup _{\epsilon>0} \sup _{x \in \Omega_{\epsilon}} \eta_{-t_{0}}^{\epsilon}(x)=C<\infty .
$$

We will not make other assumptions on the initial configuration, in particular we do not require any limit behavior as $\epsilon \rightarrow 0$. Notice however that we will study the true process only for $t \geqslant 0$ so that the true initial state at time $t=0$ will inherit some smoothness properties from the free evolution acting in the time interval $\left[-t_{0}, 0\right]$. In this sense the dependence on $\epsilon$ is "natural" and not super-imposed from the exterior.

The probability $\mu^{\epsilon}$ at time 0 is defined as the law at time $t_{0}$ of the free process which starts from $\left(\eta_{-t_{0}}^{\epsilon}, \xi_{-t_{0}}^{\epsilon}\right)$ and has generator $\epsilon^{-2} L^{(\eta, 0)}+\epsilon^{-2 a} L^{(\xi, \text { st })}$, where $L^{(\eta, 0)}$ is defined in (2.1) while $L^{(\xi, \text { st) }}$ in (2.3). It is well established in the literature, see for instance [4], that $\mu^{\epsilon}$ is a "local equilibrium measure", namely, to leading order in $\epsilon$, it is a product of Poisson measures on the $\eta$ 's and product of measures on the $\xi$ 's. Moreover, the averages of the $\eta$ variables change smoothly on the scale $\epsilon^{-1}$, while the scale of the $\xi$ particles is $\epsilon^{-a}$, see Theorem 5.1 below. We define

$$
U_{0}^{\epsilon}(x)=\mu^{\epsilon}\left(u^{\epsilon}(x, \eta)\right), \quad V_{0}^{\epsilon}(x)=\mu^{\epsilon}\left(v^{\epsilon}(x, \xi)\right)
$$

and denote by $\nabla$ the lattice gradient:

$$
e \cdot \nabla f(x)=f(x+e)-f(x), \quad|e|=1 .
$$

The following theorem is proved in Appendix A for the $\eta$ particles and in Appendix B for the $\xi$ 's.

Theorem 5.1. The initial law $\mu^{\epsilon}$ of $\left(\eta_{0}, \xi_{0}\right)$ is such that

$$
\begin{aligned}
& \lim _{\epsilon \rightarrow 0} \mu^{\epsilon}\left(\left\|u^{\epsilon}(\eta)-U_{0}^{\epsilon}\right\|^{2}+\left\|v^{\epsilon}(\xi)-V_{0}^{\epsilon}\right\|^{2}\right)=0, \\
& \sup _{\epsilon>0} \sup _{x \in \Omega_{\epsilon}}\left(U_{0}^{\epsilon}(x)+\epsilon^{-1}\left|\nabla U_{0}^{\epsilon}(x)\right|\right)<\infty, \quad \sup _{\epsilon>0} \sup _{x \in \Omega_{\epsilon}} \epsilon^{-a}\left|\nabla V_{0}^{\epsilon}(x)\right|<\infty
\end{aligned}
$$

with $\nabla$ defined in (5.3).

\section{Main results}

We suppose the law $\mu^{\epsilon}$ of $\left(\eta_{0}, \xi_{0}\right)$ (at time 0 ) as specified in the previous section and denote by $P_{\mu^{\epsilon}}^{\epsilon}$ and $E_{\mu^{\epsilon}}^{\epsilon}$ law and expectation of the process $\left(\eta_{t}, \xi_{t}\right)_{t \geqslant 0}$ with generator $L$ which starts from $\mu^{\epsilon}$. By default, in the sequel $a, \alpha$ and $\beta$ satisfy the inequalities stated at the end of Section 4. In the sequel $U^{\epsilon}$ and $V^{\epsilon}$ denote the solutions of (3.1) with initial data as in (5.2).

Theorem 6.1. There are a $2 \times 2$ matrix $A$ with constant, positive entries and a two vector $R^{\epsilon}(t)$ whose positive components depend on $\left(\eta_{s}, \xi_{s}\right)_{s \leqslant t}$, so that, for any $t \geqslant 0$, 


$$
\begin{aligned}
& \left(\begin{array}{l}
\left\|u^{\epsilon}\left(\eta_{t}\right)-U^{\epsilon}(t)\right\|^{2} \\
\left\|v^{\epsilon}\left(\xi_{t}\right)-V^{\epsilon}(t)\right\|^{2}
\end{array}\right) \leqslant\left(\begin{array}{l}
\left\|u^{\epsilon}\left(\eta_{0}\right)-U^{\epsilon}(0)\right\|^{2} \\
\left\|v^{\epsilon}\left(\xi_{0}\right)-V^{\epsilon}(0)\right\|^{2}
\end{array}\right)+\int_{0}^{t} A\left(\begin{array}{c}
\left\|u^{\epsilon}\left(\eta_{s}\right)-U^{\epsilon}(s)\right\|^{2} \\
\left\|v^{\epsilon}\left(\xi_{s}\right)-V^{\epsilon}(s)\right\|^{2}
\end{array}\right) \mathrm{d} s+R^{\epsilon}(t), \\
& \lim _{\epsilon \rightarrow 0} E_{\mu^{\epsilon}}^{\epsilon}\left(\sup _{s \leqslant t} R^{\epsilon}(s)\right)=0 .
\end{aligned}
$$

As a corollary of Theorems 5.1 and 6.1, there is $C>0$ so that, for any $\delta>0$ and any $T>0$,

$$
\lim _{\epsilon \rightarrow 0} P_{\mu^{\epsilon}}^{\epsilon}\left(\sup _{t \leqslant T}\left\{\left\|u^{\epsilon}\left(\eta_{t}\right)-U^{\epsilon}(t)\right\|^{2}+\left\|v^{\epsilon}\left(\xi_{t}\right)-V^{\epsilon}(t)\right\|^{2}\right\} \leqslant \mathrm{e}^{C T} \delta\right)=1
$$

which proves that the random fields $\left(u^{\epsilon}\left(\eta_{t}\right), v^{\epsilon}\left(\xi_{t}\right)\right)$ become deterministic as $\epsilon \rightarrow 0$ approaching the same limit behavior as $\left(U^{\epsilon}(t), V^{\epsilon}(t)\right)$ which is described by a "two scale" reaction diffusion system as we are going to see. Define

$$
U_{m}^{\epsilon}(r, t):=U^{\epsilon}\left(\epsilon^{-1} r, t\right), \quad V_{m}^{\epsilon}(r, t):=V^{\epsilon}\left(\epsilon^{-1} r, t\right), \quad r \in \epsilon \mathbb{Z}^{d} \cap \Omega
$$

$m$ standing for macroscopic as $U_{m}^{\epsilon}$ and $V_{m}^{\epsilon}$ are simply the old $U^{\epsilon}$ and $V^{\epsilon}$ re-expressed in macroscopic (un-stretched) coordinates. (3.1) then becomes

$$
\begin{aligned}
\frac{\mathrm{d} U_{m}^{\epsilon}}{\mathrm{d} t} & =\Delta_{\epsilon} U_{m}^{\epsilon}+\kappa U_{m}^{\epsilon}-F_{1}^{-}\left(U_{m}^{\epsilon}\right)-F_{2}^{-}\left(U_{m}^{\epsilon}\right) V_{m}^{\epsilon}, \\
\frac{\mathrm{d} V_{m}^{\epsilon}}{\mathrm{d} t} & =\epsilon^{2-2 a} \mathrm{e}^{-U_{m}^{\epsilon}} \Delta_{\epsilon} V_{m}^{\epsilon}+\kappa^{\prime} V_{m}^{\epsilon}\left(1-V_{m}^{\epsilon}\right) \mathrm{e}^{-U_{m}^{\epsilon}}-V_{m}^{\epsilon} G^{-}\left(U_{m}^{\epsilon}\right),
\end{aligned}
$$

where $\Delta_{\epsilon}$ is the discrete Laplacian on $\epsilon \mathbb{Z}^{d} \cap \Omega$, namely $\Delta_{\epsilon} f(r)=\epsilon^{-2} \sum_{i=1}^{d}\left[f\left(r+\epsilon e_{i}\right)-f(r)\right], e_{i}$ the unit vector in the positive $i$ th coordinate direction. It is indeed tempting by looking at (6.3) to conclude that the limit as $\epsilon \rightarrow 0$ is simply obtained by putting $\epsilon=0$ in (6.3) and by replacing $\Delta_{\epsilon}$ by the true Laplacian $\Delta$. Of course this requires that $\Delta_{\epsilon} V_{m}^{\epsilon}$ is suitably bounded. This is the case if the initial law $\mu^{\epsilon}$ makes $V_{m}^{\epsilon}$ smooth. More precisely suppose that there are smooth functions $U_{0}(r)$ and $V_{0}(r)$ such that

$$
\lim _{\epsilon \rightarrow 0}\left(\left\|U_{m}^{\epsilon}(\cdot, 0)-U_{0}(\cdot)\right\|+\left\|V_{m}^{\epsilon}(\cdot, 0)-V_{0}(\cdot)\right\|\right)=0 .
$$

In such a case (6.4) holds as well for any $t>0$ with $U(r, t)$ and $V(r, t)$ solutions of (6.3) with $\epsilon=0$ (i.e. on the torus with the true Laplacian) and with initial data $U_{0}(r)$ and $V_{0}(r)$. This is the same degenerate reaction-diffusion system derived in [10], degeneracy refers to the fact that there is no Laplacian left in the equation for $V$. Written more explicitly the equation for $V$ is

$$
\frac{\mathrm{d} V(r, t)}{\mathrm{d} t}=\kappa^{\prime} V(r, t)(1-V(r, t)) \mathrm{e}^{-U(r, t)}-V(r, t) G^{-}(U(r, t)) .
$$

Here $r$ appears as a parameter: for each $r$ we have an ordinary differential equation depending on an "external function" $U(r, t)$. Thus the solution at time $t$ will be a functional of $V_{0}(r)$ and of $U(r, s), 0 \leqslant s \leqslant t$. The equation for $U(r, t)$ then becomes:

$$
\frac{\mathrm{d} U}{\mathrm{~d} t}=\Delta U+\kappa U-F_{1}^{-}(U)-F_{2}^{-}(U) V
$$

where $V$ in (6.6) is the functional of $U$ and $V_{0}(\cdot)$ defined above.

Let us now turn to the general case of initial data as in Section 5 and drop hereafter the assumption (6.4). In such a general setup there is no reason to expect convergence as $\epsilon \rightarrow 0$, but as we will see convergence can be regained by going to subsequences without any extra assumption.

The limit evolution. As in the smooth case we have a family of equations for $V$ parameterized by $r$, however for each $r$ we do not have an ordinary differential equation but a true PDE:

$$
\frac{\mathrm{d} V\left(r^{\prime}, t\right)}{\mathrm{d} t}=\mathrm{e}^{-U(r, t)} \Delta V\left(r^{\prime}, t\right)+\kappa^{\prime} V\left(r^{\prime}, t\right)\left[1-V\left(r^{\prime}, t\right)\right] \mathrm{e}^{-U(r, t)}-V\left(r^{\prime}, t\right) G^{-}(U(r, t)) .
$$

As before the equation depends on the "external function" $U(r, \cdot)$ and its solution defines a functional of $U(r, \cdot)$ which will then be inserted in the equation for $U$. The real question however is the initial datum for (6.7). If we suppose 
that (6.4) holds then we should supplement (6.7) with the initial condition $V\left(r^{\prime}, 0\right)=V_{0}(r)$ for all $r^{\prime}$. The solution $V\left(r^{\prime}, t\right)$ is then independent of $r^{\prime}$ and we recover (6.5).

In the general case the initial datum for (6.7) is random, its distribution is given by a probability $\pi_{r}(\mathrm{~d} V)$. The origin of such a probability and its properties will be discussed in a while, we just mention here that $\pi_{r}(\mathrm{~d} V)$ is the frequency of appearance of the profile $V$, the statistics referring to a macroscopically small neighborhood of $r$. Under the validity of (6.4) the statistics is trivial: profiles around $r$ look more and more flat (constant) as $\epsilon \rightarrow 0$ so that the limit distribution $\pi_{r}(\mathrm{~d} V)$ is a Dirac delta on the constant function $V\left(r^{\prime}\right)=V_{0}(r)$. In the general setup of Section 5 oscillations on the scale $\epsilon^{1-a}$ may survive and they will be described in the limit by the measure $\pi_{r}(\mathrm{~d} V)$.

Call

$$
W(r, t ; U(r, \cdot))=\int V\left(r, t \mid V_{0}, U(r, \cdot)\right) \pi_{r}\left(\mathrm{~d} V_{0}\right),
$$

where $V\left(r, t \mid V_{0}, U(r, \cdot)\right)$ is the solution of (6.7) with initial datum $V_{0}$; the solution will of course depend on $U(r, s)$, $0 \leqslant s \leqslant t$, as indicated by the notation. We would need to prove that $V\left(r, t \mid V_{0}, U(r, \cdot)\right)$ depends measurably on $V_{0}$ so that the integral in (6.8) is well defined and this requires some regularity properties on $U$. The equation for $U$ is then, analogously to (6.6),

$$
\frac{\mathrm{d} U}{\mathrm{~d} t}=\Delta U+\kappa U-F_{1}^{-}(U)-F_{2}^{-}(U) W .
$$

We have to make sure that the solution of (6.9) is sufficiently regular as required earlier so that the "circle closes".

The analysis of all such issues is a little off the purposes of this paper and we will be very sketchy and omit all proofs. We start with the definition of the family $\pi_{r}(\mathrm{~d} V)$ which is based on the Young theorem on Young measures. We are thus in the setup of Section 5 and have:

Theorem 6.2. For any sequence $\epsilon^{\prime} \rightarrow 0$ there is a subsequence $\epsilon \rightarrow 0$ so that the following holds.

- There exists a bounded, Lipschitz function $U_{0}(r), r \in \Omega$, such that

$$
\lim _{\epsilon \rightarrow 0} \sup _{x \in \Omega_{\epsilon}}\left|U_{0}^{\epsilon}(x)-U_{0}(\epsilon x)\right|=0 .
$$

- For each $r \in \Omega$, there is a translational invariant probability measure $\pi_{r}$ on the space of [0,1]-valued functions $V$ on $\mathbb{R}^{d}$ which are uniformly Lipschitz, such that for any positive integer $n$, any smooth function $F$ on $\mathbb{R}^{n}$ and any test functions $\phi, \phi_{i}, i=1, \ldots, n, \int F\left(\ldots, \phi_{i} * V(0), \ldots\right) \pi_{r}(\mathrm{~d} V)$ is a measurable function of $r$ and

$$
\lim _{\epsilon \rightarrow 0} \epsilon^{d} \sum_{x \in \Omega_{\epsilon}} \phi(\epsilon x) F\left(\ldots, \epsilon^{a d} \sum_{y} \phi_{i}\left(\epsilon^{a} y\right) V_{0}^{\epsilon}(x+y), \ldots\right)=\int_{\Omega} \phi(r) \int F\left(\ldots, \phi_{i} * V(0), \ldots\right) \pi_{r}(\mathrm{~d} V) .
$$

In the sequel we will study the limit as $\epsilon \rightarrow 0$ of the process $\left(u^{\epsilon}\left(\eta_{t}\right), v^{\epsilon}\left(\xi_{t}\right)\right)_{t \geqslant 0}$ along a subsequence which converges at time 0 in the sense of Theorem 6.2 and $\epsilon \rightarrow 0$ will always mean "limit along such a subsequence".

Theorem 6.3. There is a unique smooth function $U(r, t)$ which solves (6.9) with $W$ as in (6.8). Moreover, if $a$, $\alpha$ and $\beta$ are as in Theorem 6.1, then for any $t$ and $\delta$ positive, any positive integer n, any bounded, smooth function $F$ on $\mathbb{R}_{+}^{n}$ and any test functions $\phi, \phi_{1}, \ldots, \phi_{n}$,

$$
\begin{aligned}
& \lim _{\epsilon \rightarrow 0} \epsilon^{d} \sum_{x \in \Omega_{\epsilon}} \phi(\epsilon x) U^{\epsilon}(x, t)=\int_{\Omega} \phi(r) U(r, t) \mathrm{d} r, \\
& \lim _{\epsilon \rightarrow 0} \epsilon^{d} \sum_{x \in \Omega_{\epsilon}} \phi(\epsilon x) F\left(\ldots, \epsilon^{a d} \sum_{y} \phi_{i}\left(\epsilon^{a} y\right) V^{\epsilon}(x+y, t), \ldots\right)=\int_{\Omega} \phi(r) \int F\left(\ldots, \phi_{i} * V(0, t), \ldots\right) \pi_{r}(\mathrm{~d} V) .
\end{aligned}
$$




\section{Scheme of proof}

The proof of Theorem 6.1 is based on the analysis of the two martingale relations (4.4), (4.5) and it is proved in Appendix C and Appendix F. The proof is in a sense computational, as we need to compute the "two compensators", namely the two integrals on the left-hand side of (4.4), (4.5). They can be written as a sum of many terms and the whole matter of the proof is to show that they fall in two categories. The first one is made by elements which are bounded by time integrals of $\left\|u^{\epsilon}\left(\eta_{t}\right)-U^{\epsilon}(t)\right\|^{2}$ or $\left\|v^{\epsilon}\left(\eta_{t}\right)-V^{\epsilon}(t)\right\|^{2}$, multiplied by coefficients which are uniformly bounded. These terms then contribute to the integral in (6.1). All the other terms must be proved to vanish as $\epsilon \rightarrow 0$, so that they contribute to the error term $R^{\epsilon}(t)$ in (6.1). Some of the estimates are straightforward but others are not trivial. The conceptually more delicate and interesting problems which arise are "anticipated" and given in the next sections, we outline in this section their typology and the way they can be analyzed.

A common feature to the analysis of all terms, is to control the large values of the variables $\eta$. A-priori $L^{\infty}$ bounds are derived in Section 8, where we show uniform in $\epsilon$ integrability of exponential moments $\mathrm{e}^{b \eta_{t}(x)}$, for any $b>0$. The result is quite standard as the process can be stochastically bounded by one having only linear births. More subtle is another bound that we use extensively in the proofs, namely that the probability that $u^{\epsilon}\left(x, \eta_{t}\right)$ exceeds a suitably large value $M=M(t)$ (but independent of $\epsilon$ ) vanishes as $\epsilon \rightarrow 0$. The proof of these statements is given in Appendix A, where we also recall from the literature results on independent random walks and random walks with independent branchings.

The other general ingredient, common to many of the proofs, is regularity in space of $u^{\epsilon}$ and $v^{\epsilon}$. In Section 8 we prove $H_{1}^{2}$ bounds uniform in $\epsilon$ which are obtained by mimicking the PDE proofs for the limit equations. Besides regularity in space we also need regularity in time of $u^{\epsilon}$. A result, maybe not optimal, but good enough for our applications, is proved in Section 9.

Such regularity estimates are the main subroutines we use to bound the "two compensators" (4.4) and (4.5). The detailed classification of all the terms which appear when computing explicitly the two compensators is reported in Appendix C. This is just some simple, but lengthy algebra, not at all deep, but necessary for the proof of Theorem 6.1, the compromise was to shift the computations to an appendix. Most of the terms in this expansion can be directly bounded using the boundedness and regularity estimates mentioned above, the bounds being uniform in $\epsilon$ and over compact time intervals. There are however some terms which do not fit in such an "easy class". The origin of the problem is the typical one found when deriving non linear hydrodynamical equations, where one needs to identify averages of non linear microscopic observables in terms of the parameters of the limit equation: in our case we find local functions of $\eta_{t}$ and of $\xi_{t}$ and we need to express them in terms of (generally different) functions of $u^{\epsilon}\left(\eta_{t}\right)$ and $v^{\epsilon}\left(\xi_{t}\right)$, (which is easy if the functions are linear). The crucial point is that these non linear terms appear in the form of time and space averages and we will solve the problem by proving local ergodic properties of the process, reminiscent of the well known "one block estimates" in the theory of hydrodynamic limits. The "two block estimates" are here replaced by the $H_{1}^{2}$ regularity already mentioned. The one block estimates are not proved using Dirichlet forms, but closeness of the process in short time intervals to a process with no deaths and births. The main difficulty here is that the $\xi$ process reminds of but it is not the stirring process, because the $\xi$ particles are allowed to jump only on sites where no $\eta$ particles are present. The local ergodic averages for the $\eta$ particles are easier to study, their analysis is reported in Section 10. The result for the $\xi$ particles is instead given in Section 11. The core of the proof is to show a homogenization property for which the $\xi$ particles move feeling to main order only the empirical average of the $\eta$ 's. The real difficulty is to prove that such a property extends to such long times for the stirring to reach local equilibrium.

\section{Boundedness and regularity in space}

In this section we will prove $L^{\infty}$ and $H_{1}^{2}$ a priori bounds which will be extensively used in the proofs of Theorem 6.1. We start from the former, which are uniform bounds on the expectations of $\eta_{t}$ and $u^{\epsilon}\left(\eta_{t}\right)$ :

Theorem 8.1. There is a constant $C^{\prime}$ so that for any $b>0$ and any $t \geqslant 0$

$\sup _{\epsilon>0} \sup _{x \in \Omega_{\epsilon}} E_{\mu^{\epsilon}}^{\epsilon}\left(\mathrm{e}^{b \eta_{t}(x)}\right) \leqslant \exp \left\{\left[\mathrm{e}^{b}-1\right] \mathrm{e}^{2 \kappa t} C^{\prime}\right\}$, 
$\kappa$ as in (2.2). Moreover, for any $\tau>0$ there are $M$ and $c$ so that

$$
\sup _{t \leqslant \tau} \sup _{x \in \Omega_{\epsilon}} P_{\mu^{\epsilon}}^{\epsilon}\left(u^{\epsilon}\left(x, \eta_{t}\right) \geqslant M\right) \leqslant c \epsilon^{(1-\alpha) d} .
$$

Proof. Let $\left(\eta_{t}^{+}\right)_{t \geqslant 0}$ be the process with generator $\epsilon^{-2} L^{(\eta, 0)}+L^{(\eta,+)}$ which starts from $\mu^{\epsilon}$. Then $\eta_{t}^{+}$dominates $\eta_{t}$, namely there is a coupling between $\left(\eta_{t}^{+}\right)_{t \geqslant 0}$ and the original process $\left(\eta_{t}, \xi_{t}\right)_{t \geqslant 0}$ (defined by the generator $L$, see (2.1)) such that $\eta_{0}^{+}=\eta_{0}$ and $\eta_{t}^{+}(x) \geqslant \eta_{t}(x)$ for all $x \in \Omega_{\epsilon}$ and all $t>0$. By an abuse of notation we still denote by $P_{\mu^{\epsilon}}^{\epsilon}$ and $E_{\mu^{\epsilon}}^{\epsilon}$, law and expectation w.r.t. the coupled process. In Appendix A it is proved that

$$
\sup _{\epsilon>0} \sup _{x \in \Omega_{\epsilon}} E_{\mu^{\epsilon}}^{\epsilon}\left(\mathrm{e}^{b \eta_{t}^{+}(x)}\right) \leqslant 2 \exp \left\{\left[\mathrm{e}^{b}-1\right] \mathrm{e}^{\kappa t}(C+2 c \sqrt{t})\right\},
$$

where $C$ is as in (5.1) and $c$ is a constant. Since $\mathrm{e}^{b \eta_{t}(x)} \leqslant \mathrm{e}^{b \eta_{t}^{+}(x)},(8.1)$ follows from (8.3).

In Appendix $\mathrm{A}$ it is also proved that

$$
\sup _{t \leqslant \tau} \sup _{x \in \Omega_{\epsilon}} E_{\mu^{\epsilon}}^{\epsilon}\left(\left[u^{\epsilon}\left(x, \eta_{t}^{+}\right)-E_{\mu^{\epsilon}}^{\epsilon}\left(u^{\epsilon}\left(x, \eta_{t}^{+}\right)\right)\right]^{2}\right) \leqslant c \epsilon^{(1-\alpha) d} .
$$

Let $M=2 \mathrm{e}^{2 \kappa \tau} C^{\prime}$, then, since $E_{\mu^{\epsilon}}^{\epsilon}\left(u^{\epsilon}\left(x, \eta_{t}^{+}\right)\right) \leqslant \mathrm{e}^{2 \kappa t} C^{\prime}$,

$$
P_{\mu^{\epsilon}}^{\epsilon}\left(u^{\epsilon}\left(x, \eta_{t}^{+}\right) \geqslant M\right) \leqslant P_{\mu^{\epsilon}}^{\epsilon}\left(\left|u^{\epsilon}\left(x, \eta_{t}^{+}\right)-E_{\mu^{\epsilon}}^{\epsilon}\left(u^{\epsilon}\left(x, \eta_{t}^{+}\right)\right)\right| \geqslant \mathrm{e}^{2 \kappa \tau} C^{\prime}\right)
$$

so that

$$
P_{\mu^{\epsilon}}^{\epsilon}\left(u^{\epsilon}\left(x, \eta_{t}^{+}\right) \geqslant M\right) \leqslant \frac{4 c}{M^{2}} \epsilon^{(1-\alpha) d} .
$$

Since $u^{\epsilon}\left(x, \eta_{t}\right) \leqslant u^{\epsilon}\left(x, \eta_{t}^{+}\right)$, (8.2) follows from (8.5).

We will next prove bounds on the $H_{1}^{2}$ norm of $u^{\epsilon}$, which will play the role of the "two blocks estimates" in the language of hydrodynamic limit theory. Before stating definition and results, let us recall how similar bounds are obtained for the heat equation $u_{t}=\Delta u$ in the unit torus $\Omega$. The "entropy" $\int_{\Omega} u^{2} \mathrm{~d} r$ gives

$$
\int_{\Omega} u^{2}(t) \mathrm{d} r-\int_{\Omega} u^{2}(0) \mathrm{d} r=-2 \int_{0}^{t} \int_{\Omega}|\nabla u|^{2} \mathrm{~d} r .
$$

Then, supposing $\int_{\Omega} u^{2}(0) \mathrm{d} r<\infty$, for any $t>0$,

$$
\int_{0}^{t} \int_{\Omega}|\nabla u|^{2} \mathrm{~d} r<\frac{1}{2} \int_{\Omega} u^{2}(0) \mathrm{d} r .
$$

The proof of Theorem 8.2 below mimics the above argument, but let us first introduce some notation and definitions which translate to the lattice the analogous notions in the continuum. If $f$ is a function on $\Omega_{\epsilon}$, we write

$$
\|f\|_{H_{1}^{2}}^{2}:=\epsilon^{-2}\|\nabla f\|^{2}
$$

with $\nabla f$ the lattice gradient of $f$, which has been defined in (5.3). We also recall that the same rules as in the continuum hold as well for the discrete gradient and Laplacian. Namely, denoting by $E_{+}$the set of unit vectors $e$ with positive components, we have, recalling (5.3) for notation and resisting to the temptation of writing $(-e) \cdot \nabla f=$ $-(e \cdot \nabla f)$, which is false,

$$
\begin{aligned}
& \Delta f(x)=\sum_{e \in E_{+}}[(-e) \cdot \nabla+e \cdot \nabla] f(x)=-\sum_{e \in E_{+}}\{(-e) \cdot \nabla\}\{(e \cdot \nabla)\} f(x), \\
& \langle g, e \cdot \nabla f\rangle=\langle(-e) \cdot \nabla g, f\rangle, \quad\langle g, \Delta f\rangle=-\langle\nabla g, \nabla f\rangle,
\end{aligned}
$$

the last equality following from the second one in (8.7) and the first one in (8.8). 
Theorem 8.2. For any $t>0$, there is $c$ so that

$$
\sup _{\epsilon>0} E_{\mu^{\epsilon}}^{\epsilon}\left(\int_{0}^{t}\left\|u^{\epsilon}\left(\eta_{s}\right)\right\|_{H_{1}^{2}}^{2} \mathrm{~d} s\right) \leqslant c .
$$

Proof. We start from the martingale relation:

$$
\left\|u^{\epsilon}\left(\eta_{t}\right)\right\|^{2}=\left\|u^{\epsilon}\left(\eta_{0}\right)\right\|^{2}+\int_{0}^{t} L\left\|u^{\epsilon}\left(\eta_{s}\right)\right\|^{2} \mathrm{~d} s+M_{t}^{\epsilon}, \quad E_{\mu^{\epsilon}}^{\epsilon}\left(M_{t}^{\epsilon}\right)=0
$$

After a simple computation which exploits the fact that discrete gradient and Laplacian satisfy the same relations as in the continuum, see (8.7), (8.8),

$$
\begin{aligned}
& \epsilon^{-2} L^{(\eta, 0)}\left\|u^{\epsilon}\right\|^{2}=-2\left\|u^{\epsilon}\right\|_{H_{1}^{2}}^{2}+R_{1}^{\epsilon}(\eta), \\
& R_{1}^{\epsilon}(\eta)=2 \epsilon^{d} \sum_{x, z} \epsilon^{-2}\left|\nabla p_{\alpha}(x, z)\right|^{2} \eta(z), \\
& L^{(\eta,+)}\left\|u^{\epsilon}\right\|^{2}=2 \kappa\left\|u^{\epsilon}\right\|^{2}+R_{2}^{\epsilon}(\eta), \quad L^{(\eta,-)}\left\|u^{\epsilon}\right\|^{2} \leqslant R_{5}^{\epsilon}(\eta, \xi), \\
& R_{2}^{\epsilon}(\eta)=\kappa \epsilon^{d} \sum_{x} \sum_{z} p_{\alpha}(x, z)^{2} \eta(z), \\
& R_{5}^{\epsilon}(\eta, \xi)=\epsilon^{d} \sum_{x} \sum_{z} p_{\alpha}(x, z)^{2} \eta(z)\left[\kappa_{\eta(z)}+\xi(z)\left(\kappa_{\eta(z)+1}-\kappa_{\eta(z)}\right)\right]
\end{aligned}
$$

(the seemingly random labelling of the remainder terms $R_{i}^{\epsilon}$ is for "historical reasons"). By taking the expectation in (8.10),

$$
2 E_{\mu^{\epsilon}}^{\epsilon}\left(\int_{0}^{t}\left\|u^{\epsilon}\left(\eta_{s}\right)\right\|_{H_{1}^{2}}^{2} \mathrm{~d} s\right) \leqslant E_{\mu^{\epsilon}}^{\epsilon}\left(\left\|u^{\epsilon}\left(\eta_{0}\right)\right\|^{2}\right)+E_{\mu^{\epsilon}}^{\epsilon}\left(\int_{0}^{t} R_{1}^{\epsilon}\left(\eta_{s}\right)+R_{2}^{\epsilon}\left(\eta_{s}\right)+R_{5}^{\epsilon}\left(\eta_{s}, \xi_{s}\right)+2 \kappa\left\|u^{\epsilon}\left(\eta_{s}\right)\right\|^{2} \mathrm{~d} s\right) .
$$

By (8.1) there is $c_{1}=c_{1}(t)$, independent of $\epsilon$, so that the right-hand side of (8.14) is bounded by $c_{1}(1+$ $\left.\sum_{z}\left[\epsilon^{-2}\left|\nabla p_{\alpha}(0, z)\right|^{2}+p_{\alpha}(0, z)^{2}\right]\right)$. By (4.6) it vanishes as $\epsilon \rightarrow 0$ because of the assumption $\alpha<d /(d+2)$ and (8.9) is proved.

We have a $H_{1}^{2}$ bound for $v^{\epsilon}$ as well, see Theorem 8.3 below, but we need first the following corollary of Theorem 8.2:

Corollary 8.1. For any $z \in \Omega_{\epsilon}$ and $t>0$

$$
\int_{0}^{t} \epsilon^{d} \sum_{x \in \Omega_{\epsilon}}\left|u^{\epsilon}\left(x+z, \eta_{s}\right)-u^{\epsilon}\left(x, \eta_{s}\right)\right| \leqslant \sqrt{t d} \epsilon|z|\left(\int_{0}^{t}\left\|u^{\epsilon}\left(\eta_{s}\right)\right\|_{H_{1}^{2}}^{2}\right)^{1 / 2} .
$$

Proof. For any $z$, there is "a coordinate curve" $\left\{y_{i}\right\}_{i=0, \ldots, N}$ such that $y_{0}=0, y_{N}=z$, with $e_{i}:=y_{i+1}-y_{i}$, $i=0, \ldots, N-1$, a unit vector, and $\sum\left|e_{i}\right|=\left|z_{1}\right|+\cdots+\left|z_{d}\right|$, having denoted by $z_{i}$ the $i$ th component of $z$. Then, recalling (5.3) for notation,

$$
u^{\epsilon}(x+z, \eta)-u^{\epsilon}(x, \eta)=\sum_{i} e_{i} \cdot \nabla u^{\epsilon}\left(x+y_{i}, \eta\right) .
$$

Hence 


$$
\begin{aligned}
\epsilon^{d} \sum_{x \in \Omega_{\epsilon}}\left|u^{\epsilon}(x+z, \eta)-u^{\epsilon}(x, \eta)\right| & \leqslant \sum_{i} \epsilon^{d} \sum_{x \in \Omega_{\epsilon}}\left|e_{i} \cdot \nabla u^{\epsilon}\left(x+y_{i}, \eta\right)\right| \\
& \leqslant \sqrt{d}\left(\sum_{i} \epsilon^{d} \sum_{x \in \Omega_{\epsilon}}\left|e_{i} \cdot \nabla u^{\epsilon}\left(x+y_{i}, \eta\right)\right|^{2}\right)^{1 / 2} \\
& \leqslant \sqrt{d} \epsilon|z|\left\|u^{\epsilon}(\eta)\right\|_{H_{1}^{2}}
\end{aligned}
$$

and (8.15) follows by Cauchy-Schwartz.

Theorem 8.3. For any $t>0$ there is $c$ so that

$$
\sup _{\epsilon>0} E_{\mu^{\epsilon}}^{\epsilon}\left(\int_{0}^{t} \epsilon^{-2 a}\left\|\nabla v^{\epsilon}\left(\xi_{s}\right)\right\|^{2} \mathrm{~d} s\right) \leqslant c .
$$

Proof. We will often use the following inequality:

$$
\left|\nabla \mathrm{e}^{-f}\right| \leqslant|\nabla f|, \quad \text { if } f \geqslant 0 .
$$

To prove the theorem, we start once again from a martingale relation:

$$
\left\|v^{\epsilon}\left(\xi_{t}\right)\right\|^{2}=\left\|v^{\epsilon}\left(\xi_{0}\right)\right\|^{2}+\int_{0}^{t} L\left\|v^{\epsilon}\left(\xi_{s}\right)\right\|^{2} \mathrm{~d} s+M_{t}^{\epsilon}, \quad E_{\mu^{\epsilon}}^{\epsilon}\left(M_{t}^{\epsilon}\right)=0 .
$$

We have that

$$
\epsilon^{-2 a} L^{(\xi, 0)}\left\|v^{\epsilon}\right\|^{2}=2 \epsilon^{-2 a} \mathcal{T}^{\epsilon}+2 \mathcal{S}^{\epsilon}+2 \mathcal{C}^{\epsilon}+R_{4}^{\epsilon},
$$

where

$$
\begin{aligned}
& \mathcal{S}^{\epsilon}(\xi, \eta)=\sum_{e:|e|=1} \epsilon^{d} \sum_{x} v^{\epsilon}(x) \epsilon^{-2 a} \sum_{z}\left[e \cdot \nabla q_{\beta}(x, z)\right] \xi(z)(1-\xi(z+e))\left[\mathbf{1}_{\eta(z+e)=0}-\mathrm{e}^{-u^{\epsilon}(z+e)}\right], \\
& \mathcal{C}^{\epsilon}(\xi, \eta)=\epsilon^{-2 a} \sum_{e:|e|=1} \epsilon^{d} \sum_{x} v^{\epsilon}(x) \sum_{z} \xi(z)(1-\xi(z+e))\left[\mathrm{e}^{-u^{\epsilon}(z+e)}-\mathrm{e}^{-u^{\epsilon}(x)}\right] e \cdot \nabla q_{\beta}(x, z), \\
& R_{4}^{\epsilon}(\xi, \eta)=\epsilon^{-2 a} \sum_{e:|e|=1} \epsilon^{d} \sum_{x} \sum_{z} \xi(z)(1-\xi(z+e)) \mathbf{1}_{\eta(z+e)=0}\left(e \cdot \nabla q_{\beta}(x, z)\right)^{2} .
\end{aligned}
$$

Finally, (below $E_{+}$is the set of unit vectors in $\mathbb{Z}^{d}$ with non negative components)

$$
\begin{aligned}
\mathcal{T}^{\epsilon}:= & \epsilon^{d} \sum_{x} v^{\epsilon}(x) \mathrm{e}^{-u^{\epsilon}(x)} \sum_{e:|e|=1} \sum_{z} \xi(z)(1-\xi(z+e))[e \cdot \nabla] q_{\beta}(x, z) \\
= & \epsilon^{d} \sum_{x} v^{\epsilon}(x) \mathrm{e}^{-u^{\epsilon}(x)} \sum_{e \in E_{+}} \sum_{z} \xi(z)[(-e) \cdot \nabla+e \cdot \nabla] q_{\beta}(x, z) \\
& -\epsilon^{d} \sum_{x} v^{\epsilon}(x) \mathrm{e}^{-u^{\epsilon}(x)} \sum_{e \in E_{+}}\left\{\sum_{z}\left[\xi(z) \xi(z-e)[(-e) \cdot \nabla] q_{\beta}(x, z)+\xi(z) \xi(z+e)[e \cdot \nabla] q_{\beta}(x, z)\right]\right\} .
\end{aligned}
$$

By (8.7), in the last term the quantity in curly brackets is zero, while for the first one we have that $\sum_{e \in E_{+}} \sum_{z} \xi(z)$. $[(-e) \cdot \nabla+e \cdot \nabla] q_{\beta}(z-x)=\Delta v^{\epsilon}(x)$. Thus

$$
\epsilon^{-2 a} L^{(\xi, 0)}\left\|v^{\epsilon}\right\|^{2}=2 \epsilon^{-2 a}\left\langle v^{\epsilon}, \mathrm{e}^{-u^{\epsilon}} \Delta v^{\epsilon}\right\rangle+2 \mathcal{S}^{\epsilon}+2 \mathcal{C}^{\epsilon}+R_{4}^{\epsilon} .
$$

The proof that $\lim _{\epsilon \rightarrow 0}\left|\int_{0}^{t} E_{\mu^{\epsilon}}^{\epsilon}\left(\mathcal{S}^{\epsilon}\left(\xi_{s}, \eta_{s}\right) \mathrm{d} s\right)\right|=0$ follows from Theorem 10.1 below (details are left to Appendix D). We will use the present theorem only after Theorem 10.1, so that there is no circularity in our arguments. 
By Corollary 8.1, Theorem 8.2, (8.18) and (4.9) we then have

$$
\left|\int_{0}^{t} E_{\mu^{\epsilon}}^{\epsilon}\left(\mathcal{C}^{\epsilon}\left(\xi_{s}, \eta_{s}\right)\right) \mathrm{d} s\right| \leqslant c \epsilon^{1-2 a}
$$

By (4.7),

$$
\left|R_{4}^{\epsilon}(\xi, \eta)\right| \leqslant c \epsilon^{-2 a+2(a-\beta)+d(a-\beta)}
$$

which also vanishes in the limit $\epsilon \rightarrow 0$ because of the assumption $\beta<\frac{d}{d+2} a$. Let $\chi_{M}=\left\{\chi_{M, x}(s), x \in \Omega_{\epsilon}\right\}$ where $\chi_{M, x}(s)$ denotes the characteristic function of $\left\{u^{\epsilon}\left(x, \eta_{s}\right) \leqslant M\right\}$. We use the integration by parts formula (8.8) to write

$$
2 \epsilon^{-2 a}\left\langle\mathrm{e}^{-u^{\epsilon}} v^{\epsilon}, \Delta v^{\epsilon}\right\rangle \leqslant-2 \epsilon^{-2 a} \mathrm{e}^{-M}\left\|\nabla v^{\epsilon}\right\|^{2}+\mathcal{R}_{5}^{\epsilon}+\mathcal{R}_{6}^{\epsilon}
$$

where

$$
\mathcal{R}_{5}^{\epsilon}=-2 \epsilon^{-2 a}\left\langle v^{\epsilon}\left(\nabla \mathrm{e}^{-u^{\epsilon}}\right), \nabla v^{\epsilon}\right\rangle, \quad \mathcal{R}_{6}^{\epsilon}=-2 \epsilon^{-2 a}\left\langle\left[1-\chi_{M}\right] \nabla v^{\epsilon},\left(\mathrm{e}^{-u^{\epsilon}}-\mathrm{e}^{-M}\right) \nabla v^{\epsilon}\right\rangle .
$$

By (8.9), (8.18) and Corollary 8.1, since $\left|v^{\epsilon}\right| \leqslant 1,\left|\nabla v^{\epsilon}\right| \leqslant 2 d$

$$
\int_{0}^{t}\left|\mathcal{R}_{5}^{\epsilon}\right| \leqslant c \epsilon^{1-2 a}
$$

By (8.2), we have

$$
\sup _{s \leqslant t} E_{\mu^{\epsilon}}^{\epsilon}\left(\left|\mathcal{R}_{6}^{\epsilon}\left(\xi_{s}, \eta_{s}\right)\right|\right) \leqslant c \epsilon^{d(1-\alpha)-2 a} .
$$

We next observe that $L^{(\xi,+)}\left\|v^{\epsilon}\right\|^{2} \leqslant 2 \kappa^{\prime}\left\|v^{\epsilon}\right\|^{2}+R_{8}^{\epsilon}$ and $L^{(\xi,-)}\left\|v^{\epsilon}\right\|^{2} \leqslant R_{9}^{\epsilon}$, where

$$
\begin{aligned}
& R_{8}^{\epsilon}=\kappa^{\prime} \epsilon^{d} \sum_{x} \frac{1}{2 d} \sum_{e:|e|=1} \sum_{z} q_{\beta}(x, z+e)^{2} \xi(z)[1-\xi(z+e)] \mathbf{1}_{\eta(z+e)=0}, \\
& R_{9}^{\epsilon}=\epsilon^{d} \sum_{x} \sum_{z} q_{\beta}(x, z)^{2} \xi(z) \kappa_{\eta(z)+1} .
\end{aligned}
$$

By (4.8) and since $\kappa_{n} \leqslant c \mathrm{e}^{b n}$, then, by (8.1), also $R_{8}$ and $R_{9}$ give a vanishing contribution. We have therefore proved that there is a positive function $\varphi_{\epsilon}(t) \rightarrow 0$ as $\epsilon \rightarrow 0$ such that

$$
\int_{0}^{t} E_{\mu^{\epsilon}}^{\epsilon}\left(L^{(\xi)}\left\|v^{\epsilon}\left(\xi_{s}\right)\right\|^{2}\right) \mathrm{d} s \leqslant-2 \epsilon^{-2 a} \mathrm{e}^{-M} \int_{0}^{t} E_{\mu^{\epsilon}}^{\epsilon}\left(\left\|\nabla v^{\epsilon}\left(\xi_{s}\right)\right\|^{2}+2 \kappa^{\prime}\left\|v^{\epsilon}\left(\xi_{s}\right)\right\|^{2}\right) \mathrm{d} s+\varphi_{\epsilon}(t) .
$$

Thus from (8.19) and (8.25) we get

$$
\mathrm{e}^{-M} E_{\mu^{\epsilon}}^{\epsilon}\left(\int_{0}^{t} \epsilon^{-2 a}\left\|\nabla v^{\epsilon}\left(\xi_{s}\right)\right\|^{2} \mathrm{~d} s\right) \leqslant E_{\mu^{\epsilon}}^{\epsilon}\left(\left\|v^{\epsilon}\left(\xi_{0}\right)\right\|^{2}\right)+\kappa^{\prime} t+\varphi_{\epsilon}(t) .
$$

Theorem 8.3 is proved.

We conclude the section with the following corollary of Theorem 8.2, which will be needed in the computation of the compensators (4.3).

Corollary 8.2. For any $t>0$ there is $\bar{C}$ so that

$$
\sup _{\epsilon>0} \sup _{x \in \Omega_{\epsilon}} \sup _{s \leqslant t}\left|U^{\epsilon}(x, s)\right| \leqslant \bar{C} .
$$

Furthermore for any $t>0$ there is $c$ so that for any $s \leqslant t$ 


$$
\begin{aligned}
& \left\langle\left[v^{\epsilon}\left(\xi_{s}\right)-V^{\epsilon}(s)\right],\left[\mathrm{e}^{-u^{\epsilon}\left(\eta_{s}\right)}-\mathrm{e}^{-U^{\epsilon}(s)}\right] \epsilon^{-2 a} \Delta V^{\epsilon}(s)\right\rangle \\
& \leqslant \frac{\mathrm{e}^{-\bar{C}}}{10} \epsilon^{-2 a}\left\|\nabla\left\{v^{\epsilon}\left(\xi_{s}\right)-V^{\epsilon}(s)\right\}\right\|^{2}+c\left(\left\|u^{\epsilon}\left(\eta_{s}\right)-U^{\epsilon}(s)\right\|^{2}+\left\|v^{\epsilon}\left(\xi_{s}\right)-V^{\epsilon}(s)\right\|^{2}\right. \\
& \left.\quad+\epsilon^{2-2 a}\left\{\left\|u^{\epsilon}\left(\eta_{s}\right)\right\|_{H_{1}^{2}}^{2}+\left\|U^{\epsilon}(s)\right\|_{H_{1}^{2}}^{2}\right\}\right) .
\end{aligned}
$$

Proof. Since $U^{\epsilon}$ and $V^{\epsilon}$ are solutions of (3.1) with initial data which satisfy (5.5), by "standard arguments" (8.26) is verified and for any $t$ there is $C$ so that

$$
\sup _{\epsilon>0} \sup _{x \in \Omega_{\epsilon}, s \leqslant t} \epsilon^{-a}\left|\nabla V^{\epsilon}(x, s)\right|=C<\infty .
$$

Then, by (8.8)

$$
\begin{aligned}
\left\langle\left[v^{\epsilon}-V^{\epsilon}\right],\left[\mathrm{e}^{-u^{\epsilon}}-\mathrm{e}^{-U^{\epsilon}}\right] \epsilon^{-2 a} \Delta V^{\epsilon}\right\rangle \leqslant & C\left(\left\langle\epsilon^{-a}\left|\nabla\left\{v^{\epsilon}-V^{\epsilon}\right\}\right|,\left|\mathrm{e}^{-u^{\epsilon}}-\mathrm{e}^{-U^{\epsilon}}\right|\right\rangle\right. \\
& \left.+\left\langle\left|v^{\epsilon}-V^{\epsilon}\right|, \epsilon^{-a}\left|\nabla\left\{\mathrm{e}^{-u^{\epsilon}}-\mathrm{e}^{-U^{\epsilon}}\right\}\right|\right|\right) .
\end{aligned}
$$

Since $\left|\mathrm{e}^{-u^{\epsilon}}-\mathrm{e}^{-U^{\epsilon}}\right| \leqslant\left|u^{\epsilon}-U^{\epsilon}\right|$, using the inequality $2|d b| \leqslant \delta d^{2}+\delta^{-1} b^{2}$, with $b=\left|u^{\epsilon}-U^{\epsilon}\right|, d=\epsilon^{-a} \mid \nabla\left\{v^{\epsilon}-\right.$ $\left.V^{\epsilon}\right\} \mid$, and $\delta=\mathrm{e}^{-\bar{C}}(5 C)^{-1}$, we get

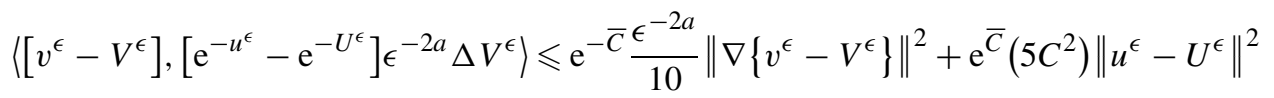

$$
\begin{aligned}
& +C\left(\left\|v^{\epsilon}-V^{\epsilon}\right\|^{2}+\frac{\epsilon^{-2 a}}{2}\left\|\nabla u^{\epsilon}\right\|^{2}+\frac{\epsilon^{-2 a}}{2}\left\|\nabla U^{\epsilon}\right\|^{2}\right)
\end{aligned}
$$

having also used (8.18). Recalling the definition of $\|f\|_{H_{1}^{2}}$ in (8.6) we then get (8.27).

\section{Regularity in time}

Besides regularity in space, we will also need estimates on the regularity of $u^{\epsilon}\left(x, \eta_{t}\right)$ as a function of the time $t$. This is needed in Section 11 and Appendix D, and the statement we will prove here is just what is used in the sequel, with no aim at generality. We denote by $P_{\eta_{t}, \xi_{t}}^{\epsilon}$ and $E_{\eta_{t}, \xi_{t}}^{\epsilon}$, conditional law and expectation of the process $\left(\eta_{s}, \xi_{s}\right)_{s} \geqslant t$, given the state $\left(\eta_{t}, \xi_{t}\right)$ at time $t$.

Theorem 9.1. For any $\tau$ and $\gamma$ positive, with $2 \gamma<(1-\alpha) d$, there is $c$ so that for any $t \leqslant \tau, \epsilon>0, x \in \Omega_{\epsilon}$ and any $s \in\left(t, t+\epsilon^{2 \gamma}\right]$

$$
E_{\mu^{\epsilon}}^{\epsilon}\left(\left|u^{\epsilon}\left(x, \eta_{s}\right)-\pi_{(s-t) \epsilon^{-2}} * u^{\epsilon}\left(x, \eta_{t}\right)\right|\right) \leqslant c \epsilon^{2 \gamma},
$$

where $\pi$ is defined in (4.1) and $\pi_{(s-t) \epsilon^{-2}} * u^{\epsilon}\left(x, \eta_{t}\right)=\sum_{y} \pi_{(s-t) \epsilon^{-2}}(x, y) u^{\epsilon}\left(y, \eta_{t}\right)$.

Proof. We define an auxiliary process $\left(\theta_{s}, \xi_{s}\right)_{s} \geqslant t, \theta_{s}=\left(\eta_{s}^{(F, a)}, \eta_{s}^{(F, d)}, \eta_{s}^{(b)}\right)$, and the following random variables on this process,

$$
\eta_{s}:=\eta_{s}^{(F, a)}+\eta_{s}^{(b)}, \quad \eta_{s}^{(F)}:=\eta_{s}^{(F, a)}+\eta_{s}^{(F, d)} .
$$

The main point of the definition will be that $\left(\eta_{s}, \xi_{s}\right)_{s \geqslant t}$ has the same law as the process of Section 2 with generator $L$, while $\eta_{s}^{(F)}$ has the law of the independent process with generator $\epsilon^{-2} L^{(\eta, 0)}$. The $\eta_{s}^{(F, a)}$ particles are called free and alive; $\eta_{s}^{(F, d)}$ free but dead; $\eta_{s}^{(b)}$ newly born, which already hints at the way the whole process will be defined. We set $\eta_{t}^{(F, d)}=\eta_{t}^{(b)}=0$, so that $\eta_{t}=\eta_{t}^{(F, a)}$. The process $\left(\theta_{s}, \xi_{s}\right)$ at times $s \geqslant t$ is defined in terms of the generator $\tilde{L}$ which we set equal to $\tilde{L}=L_{1}+L^{(\xi)}$, with $L^{(\xi)}$ as in Section 2 (reading $\eta=\eta^{(F, a)}+\eta^{(b)}$ ). $L_{1}$ is $\epsilon^{-2} L_{1}^{(0)}+L_{1}^{(+)}+L_{1}^{(-)}$. $L_{1}^{(0)}$ is the generator of independent motion for the three types of $\eta$ particles.

$$
L_{1}^{(+)} f(\theta, \xi)=\sum_{x \in \Omega_{\epsilon}} \kappa \eta(x)\left[f\left(\theta+1_{x, b}, \xi\right)-f(\theta, \xi)\right],
$$


where $1_{x, b}, 1_{x,(F, d)}$ and $1_{x,(F, a)}$ below are the configurations with only one particle at $x$ respectively of type $b,(F, d)$ and $(F, a)$;

$$
\begin{aligned}
L_{1}^{(-)} f(\theta, \xi)= & \sum_{x \in \Omega_{\epsilon}} \eta(x)\left(\kappa_{\eta(x)}(1-\xi(x))+\kappa_{\eta(x)+1} \xi(x)\right) \\
& \times\left\{\mathbf{1}_{\eta^{(b)}(x)=0}\left[f\left(\theta-1_{x,(F, a)}+1_{x,(F, d)}, \xi\right)-f(\theta, \xi)\right]+\mathbf{1}_{\eta^{(b)}(x)>0}\left[f\left(\theta-1_{x, b}, \xi\right)-f(\theta, \xi)\right]\right\} .
\end{aligned}
$$

It is then easy to check that $\left(\eta_{s}, \xi_{s}\right)$ is our original process and $\eta^{(F)}$ is the independent process.

By an abuse of notation we still denote by $E_{\mu^{\epsilon}}^{\epsilon}$ the expectation relative to the process which after time $t$ has generator $\tilde{L}$. Then, using (9.2),

$$
\begin{aligned}
& E_{\mu^{\epsilon}}^{\epsilon}\left(\mid u^{\epsilon}\left(x, \eta_{s}\right)-\pi_{\left.(s-t) \epsilon^{-2} * u^{\epsilon}\left(x, \eta_{t}\right) \mid\right)}\right. \\
& \quad \leqslant E_{\mu^{\epsilon}}^{\epsilon}\left(\left|u^{\epsilon}\left(x, \eta_{s}^{(F)}\right)-\pi_{(s-t) \epsilon^{-2}} * u^{\epsilon}\left(x, \eta_{t}\right)\right|\right)+E_{\mu^{\epsilon}}^{\epsilon}\left(u^{\epsilon}\left(x, \eta_{s}^{(b)}\right)+u^{\epsilon}\left(x, \eta_{s}^{(F, d)}\right)\right) .
\end{aligned}
$$

Recalling that $u^{\epsilon}\left(x, \eta_{t}^{(b)}\right)=u^{\epsilon}\left(x, \eta_{t}^{(F, d)}\right)=0$, by (A.6),

$$
\begin{aligned}
& E_{\mu^{\epsilon}}^{\epsilon}\left(u^{\epsilon}\left(x, \eta_{s}^{(b)}\right)+u^{\epsilon}\left(x, \eta_{s}^{(F, d)}\right)\right) \\
& \quad=\int_{t}^{s} \sum_{y} \pi_{\left(s-s^{\prime}\right) \epsilon^{-2}}(x, y) E_{\mu^{\epsilon}}^{\epsilon}\left(\left(L_{1}^{(+)}+L_{1}^{(-)}\right)\left\{u^{\epsilon}\left(y, \eta_{s^{\prime}}^{(b)}\right)+u^{\epsilon}\left(y, \eta_{s^{\prime}}^{(F, d)}\right)\right\}\right) .
\end{aligned}
$$

Since

$$
\begin{aligned}
& \left(L_{1}^{(+)}+L_{1}^{(-)}\right) u^{\epsilon}\left(y, \eta^{(b)}\right) \leqslant \kappa u^{\epsilon}(y, \eta) \\
& \left(L_{1}^{(+)}+L_{1}^{(-)}\right) u^{\epsilon}\left(y, \eta^{(F, d)}\right) \leqslant \sum_{z} p_{\alpha}(y, z) \eta(z) \kappa_{\eta(z)+1}
\end{aligned}
$$

by Theorem 8.1 there is $c=c(t)$ such that

$$
E_{\mu^{\epsilon}}^{\epsilon}\left(\left|u^{\epsilon}\left(x, \eta_{s}\right)-\pi_{(s-t) \epsilon^{-2}} * u^{\epsilon}\left(x, \eta_{t}\right)\right|\right) \leqslant E_{\mu^{\epsilon}}^{\epsilon}\left(\left|u^{\epsilon}\left(x, \eta_{s}^{(F)}\right)-\pi_{(s-t) \epsilon^{-2}} * u^{\epsilon}\left(x, \eta_{t}\right)\right|\right)+c \epsilon^{2 \gamma} .
$$

Let

$$
\tilde{\eta}_{s}(y)=\eta_{s}^{(F)}(y)-\pi_{(s-t) \epsilon^{-2}} * \eta_{t}(y)
$$

then

$$
u^{\epsilon}\left(x, \eta_{s}^{(F)}\right)-\pi_{(s-t) \epsilon^{-2}} * u^{\epsilon}\left(x, \eta_{t}\right)=p_{\alpha} * \tilde{\eta}_{s}(x)
$$

and, by Cauchy-Schwartz and (4.6), the square of the first term on the right-hand side of (9.4) is bounded by

$$
E_{\mu^{\epsilon}}^{\epsilon}\left(\left|p_{\alpha} * \tilde{\eta}_{s}(x)\right|^{2}\right)=E_{\mu^{\epsilon}}^{\epsilon}\left(\sum_{y} p_{\alpha}^{2}(x, y) \tilde{\eta}_{s}(y)^{2}\right) \leqslant c^{\prime} \epsilon^{(1-\alpha) d}
$$

because $E_{\eta_{t}, \xi_{t}}^{\epsilon}\left(\tilde{\eta}_{s}(y) \tilde{\eta}_{s}(z)\right)=0$ for $z \neq y$ by (A.5)-(A.7). The last inequality in (9.5) follows from Theorem 8.1. By assumption, $(1-\alpha) d>2 \gamma$ and this concludes the proof of the theorem.

\section{Local ergodic theorems}

In this section we will prove local equilibrium in the spirit of the so called "Gibbs-Boltzmann principle" [3], and "one block estimate" [8], in the hydrodynamic limit literature. The question concerns time averages and the aim is to prove closeness to equilibrium expectations with parameters determined by local empirical means (local equilibrium). This can be reduced (the details are in Appendix D) to a proof of decay of time correlations, which is the content of Theorems 10.1 and 10.2 below, for some bounded and unbounded local functions of $\eta_{t}$.

Theorem 10.1. For any $\tau>0$ there is $c$ so that for any $\epsilon>0, x, y \in \Omega_{\epsilon},|x-y| \geqslant \epsilon^{-1+\alpha / 2}$ and $t \leqslant \tau$,

$$
\left|E_{\mu^{\epsilon}}^{\epsilon}\left(\left\{\mathbf{1}_{\eta_{t+\epsilon^{2 \alpha}}(x)=0}-\mathrm{e}^{-u^{\epsilon}\left(x, \eta_{t}\right)}\right\}\left\{\mathbf{1}_{\eta_{t+\epsilon^{2 \alpha}}(y)=0}-\mathrm{e}^{-u^{\epsilon}\left(y, \eta_{t}\right)}\right\}\right)\right| \leqslant c \epsilon^{2 \alpha} .
$$


Proof. The proof uses the same auxiliary process introduced in the beginning of the proof of Theorem 9.1, to which we refer for notation. To compute the expectation we shorthand $\mathbf{1}_{z,>>}=\mathbf{1}_{\eta_{t+\epsilon^{2 \alpha}}^{(F, d)}(z)+\eta_{t+\epsilon^{2 \alpha}}^{(b)}(z)>0}$ and since $\eta_{t+\epsilon^{2 \alpha}}^{(F)}=\eta_{t+\epsilon^{2 \alpha}}^{(F, a)}+\eta_{t+\epsilon^{2 \alpha}}^{(d)}$ and $\eta_{t+\epsilon^{2 \alpha}}=\eta_{t+\epsilon^{2 \alpha}}^{(F, a)}+\eta_{t+\epsilon^{2 \alpha}}^{(b)}$, see (9.2),

$$
\left\{\eta_{t+\epsilon^{2 \alpha}}^{(F)}(z) \neq \eta_{t+\epsilon^{2 \alpha}}(z)\right\} \subset\left\{\eta_{t+\epsilon^{2 \alpha}}^{(F, d)}(z)+\eta_{t+\epsilon^{2 \alpha}}^{(b)}(z)>0\right\} .
$$

Since $E_{\mu^{\epsilon}}^{\epsilon}\left(\mathbf{1}_{z,>}\right) \leqslant E_{\mu^{\epsilon}}^{\epsilon}\left(\eta_{t+\epsilon^{2 \alpha}}^{(F, d)}(z)+\eta_{t+\epsilon^{2 \alpha}}^{(b)}(z)\right)$ and since the curly brackets in (10.1) have absolute value $\leqslant 1$,

$$
\begin{aligned}
\text { 1.h.s. of }(10.1) \leqslant & \left|E_{\mu^{\epsilon}}^{\epsilon}\left(\left\{\mathbf{1}_{\eta_{t+\epsilon^{2 \alpha}}^{(F)}(x)=0}-\mathrm{e}^{-u^{\epsilon}\left(x, \eta_{t}\right)}\right\}\left\{\mathbf{1}_{\eta_{t+\epsilon^{2 \alpha}}^{(F)}(y)=0}-\mathrm{e}^{-u^{\epsilon}\left(y, \eta_{t}\right)}\right\}\right)\right| \\
& +\left|E_{\mu^{\epsilon}}^{\epsilon}\left(\eta_{t+\epsilon^{2 \alpha}}^{(F, d)}(x)+\eta_{t+\epsilon^{2 \alpha}}^{(b)}(x)+\eta_{t+\epsilon^{2 \alpha}}^{(F, d)}(y)+\eta_{t+\epsilon^{2 \alpha}}^{(b)}(y)\right)\right| .
\end{aligned}
$$

Recalling that by definition $\eta_{t}^{(F, d)}=\eta_{t}^{(b)}=0$, then, similarly to (9.3),

$$
E_{\mu^{\epsilon}}^{\epsilon}\left(\eta_{t+\epsilon^{2 \alpha}}^{(F, d)}(z)+\eta_{t+\epsilon^{2 \alpha}}^{(b)}(z)\right) \leqslant c \epsilon^{2 \alpha}
$$

and (10.3) yields

$$
\text { 1.h.s. of }(10.1) \leqslant\left|E_{\mu^{\epsilon}}^{\epsilon}\left(\left\{\mathbf{1}_{\eta_{t+\epsilon^{2 \alpha}}^{(F)}(x)=0}-\mathrm{e}^{-u^{\epsilon}\left(x, \eta_{t}\right)}\right\}\left\{\mathbf{1}_{\eta_{t+\epsilon^{2 \alpha}}^{(F)}(y)=0}-\mathrm{e}^{-u^{\epsilon}\left(y, \eta_{t}\right)}\right\}\right)\right|+c \epsilon^{2 \alpha} \text {. }
$$

Call $\eta_{t, x}(z)=\eta_{t}(z) \mathbf{1}_{|z-x| \leqslant \epsilon^{-1+\alpha / 2} / 4}$ and $\eta_{t+\epsilon^{2 \alpha}, x}^{(F)}$ the free evolution starting from $\eta_{t, x} . \eta_{t, y}$ and $\eta_{t+\epsilon^{2 \alpha, y}}^{(F)}$ are defined analogously.

$$
\begin{aligned}
& \left|E_{\mu^{\epsilon}}^{\epsilon}\left(\left\{\mathbf{1}_{\eta_{t+\epsilon^{2 \alpha}}^{(F)}(x)=0}-\mathrm{e}^{-u^{\epsilon}\left(x, \eta_{t}\right)}\right\}\left\{\mathbf{1}_{\eta_{t+\epsilon^{2 \alpha}}^{(F)}(y)=0}-\mathrm{e}^{-u^{\epsilon}\left(y, \eta_{t}\right)}\right\}\right)\right| \\
& \leqslant \mid \\
& \quad+E_{\mu^{\epsilon}}^{\epsilon}\left(\{ \mathbf { 1 } _ { \eta _ { t + \epsilon ^ { 2 \alpha } , x } ^ { ( F ) } ( x ) = 0 } - \mathrm { e } ^ { - u ^ { \epsilon } ( x , \eta _ { t , x } ) } \} \left\{\mathbf{1}_{\eta_{t+\epsilon^{2 \alpha}, y}^{(F)}}(y)=0\right.\right. \\
& \quad+E_{\mu^{\epsilon}}^{\epsilon}\left(\left\{\sum_{|z-x|>\epsilon^{-1+\alpha / 2} / 4} \eta_{t}(z) \pi_{\epsilon^{-2+2 \alpha}}(z, x)\right\}\right)+E_{\mu^{\epsilon}}^{\epsilon}\left(\left\{\sum_{|z-y|>\epsilon^{-1+\alpha / 2} / 4} \eta_{t}(z) \pi_{\epsilon^{-2+2 \alpha}}(z, y)\right\}\right) .
\end{aligned}
$$

Then, by Theorem 8.1 and classical properties of single random walks, there are positive constants $c$ and $c^{\prime}$ so that

$$
\begin{aligned}
& \left|E_{\mu^{\epsilon}}^{\epsilon}\left(\left\{\mathbf{1}_{\eta_{t+\epsilon^{2 \alpha}}^{(F)}(x)=0}-\mathrm{e}^{-u^{\epsilon}\left(x, \eta_{t}\right)}\right\}\left\{\mathbf{1}_{\eta_{t+\epsilon^{(\alpha)}}^{(F)}(y)=0}-\mathrm{e}^{-u^{\epsilon}\left(y, \eta_{t}\right)}\right\}\right)\right| \\
& \quad \leqslant\left|E_{\mu^{\epsilon}}^{\epsilon}\left(\left\{\mathbf{1}_{\eta_{t+\epsilon^{(\alpha)}, x}(x)=0}-\mathrm{e}^{-u^{\epsilon}\left(x, \eta_{t, x}\right)}\right\}\left\{\mathbf{1}_{\eta_{t+\epsilon^{(\alpha)}, y}^{(F)}(y)=0}-\mathrm{e}^{-u^{\epsilon}\left(y, \eta_{t, y}\right)}\right\}\right)\right|+c \mathrm{e}^{-c^{\prime} \epsilon^{-\alpha}} .
\end{aligned}
$$

The conditional expectation given $\eta_{t}$ of the product of the two curly brackets factorizes and

$$
\begin{aligned}
P_{\eta_{t}}^{\epsilon}\left(\left\{\eta_{t+\epsilon^{2 \alpha}, x}^{(F)}(x)=0\right\}\right) & =\prod_{|z-x| \leqslant \epsilon^{-1+\alpha / 2} / 4}\left(1-\pi_{\epsilon^{-2+2 \alpha}}(z, x)\right)^{\eta_{t}(z)} \\
& =\exp \left\{\sum_{|z-x| \leqslant \epsilon^{-1+\alpha / 2} / 4} \eta_{t}(z) \log \left[1-\pi_{\epsilon^{-2+2 \alpha}}(z, x)\right]\right\} \\
& =\mathrm{e}^{-u^{\epsilon}\left(x, \eta_{t, x}\right)\left(1+R_{x}\right)}, \\
\left|R_{x}\right| \leqslant c \epsilon^{(1-\alpha) d} . &
\end{aligned}
$$

An analogous bound holds when $x$ is replaced by $y$, so that the left-hand side of (10.1) is bounded by

$$
\left|E_{\mu^{\epsilon}}^{\epsilon}\left(\left\{\mathrm{e}^{-u^{\epsilon}\left(x, \eta_{t, x}\right)\left(1+R_{x}\right)}-\mathrm{e}^{-u^{\epsilon}\left(x, \eta_{t, x}\right)}\right\}\left\{\mathrm{e}^{-u^{\epsilon}\left(y, \eta_{t, y}\right)\left(1+R_{y}\right)}-\mathrm{e}^{-u^{\epsilon}\left(y, \eta_{t, y}\right)}\right\}\right)\right|+c \epsilon^{2 \alpha} .
$$

We distinguish $u^{\epsilon}\left(x, \eta_{t, x}\right)>M$ and $u^{\epsilon}\left(x, \eta_{t, x}\right) \leqslant M$. The contribution of the former is bounded using Theorem 8.1, while in the latter case we bound by (8.18) $\left|\mathrm{e}^{-u^{\epsilon}\left(x, \eta_{t, x}\right)\left(1+R_{x}\right)}-\mathrm{e}^{-u^{\epsilon}\left(x, \eta_{t, x}\right)}\right| \leqslant M\left|R_{x}\right|$. Same procedure is used for the term with $y$, thus concluding the proof of $(10.1)$ because $(1-\alpha) d>2 \alpha$. 
Denoting by $\mathcal{P}_{\rho}$ and $\mathcal{E}_{\rho}$ law and expectation w.r.t. the product of identical Poisson measures with mean $\rho>0$, given a function $g=g(n), n \in \mathbb{N}$, we shorthand

$$
\begin{aligned}
f_{\epsilon}\left(\eta_{t}, \eta_{t+\epsilon^{2 \alpha}}(x), \eta_{t+\epsilon^{2 \alpha}}(y)\right):= & \mathbf{1}_{u^{\epsilon}\left(x, \eta_{t}\right) \leqslant M, u^{\epsilon}\left(y, \eta_{t}\right) \leqslant M}\left\{g\left(\eta_{t+\epsilon^{2 \alpha}}(x)\right)-\mathcal{E}_{u^{\epsilon}\left(x, \eta_{t}\right)}(g)\right\} \\
& \times\left\{g\left(\eta_{t+\epsilon^{2 \alpha}}(y)\right)-\mathcal{E}_{u^{\epsilon}}\left(y, \eta_{t}\right)(g)\right\}
\end{aligned}
$$

with $M$ as in (8.2).

Theorem 10.2. With the notation of (10.5), suppose $g(n) \leqslant \mathrm{e}^{b n}, b>0$, then, for any $\tau>0$ there is $c$ so that, for any $\epsilon>0$ and $t \leqslant \tau$,

$$
\limsup _{\epsilon \rightarrow 0}\left|E_{x \neq y}^{\epsilon}\left(f_{\epsilon}\left(\eta_{t}, \eta_{t+\epsilon^{2 \alpha}}(x), \eta_{t+\epsilon^{2 \alpha}}(y)\right)\right)\right|=0 .
$$

Proof. By (10.2) and (10.4), using Cauchy-Schwartz,

$$
\begin{aligned}
& \left|E_{\mu^{\epsilon}}^{\epsilon}\left(f_{\epsilon}\left(\eta_{t}, \eta_{t+\epsilon^{2 \alpha}}(x), \eta_{t+\epsilon^{2 \alpha}}(y)\right)\right)-E_{\mu^{\epsilon}}^{\epsilon}\left(f_{\epsilon}\left(\eta_{t}, \eta_{t+\epsilon^{2 \alpha}}^{(F)}(x) \eta_{t+\epsilon^{2 \alpha}}^{(F)}(y)\right)\right)\right| \\
& \quad \leqslant E_{\mu^{\epsilon}}^{\epsilon}\left(f_{\epsilon}^{2}\right)^{1 / 2} c \epsilon^{\alpha} \leqslant c^{\prime} \epsilon^{\alpha} .
\end{aligned}
$$

The last inequality uses first that the functions $\mathbf{1}_{u^{\epsilon}\left(z, \eta_{t}\right) \leqslant M} \mathcal{E}_{u^{\epsilon}\left(z, \eta_{t}\right)}(g), z=x, y$, are bounded and then Theorem 8.1 (as $f_{\epsilon}^{2}$ is exponentially bounded). By (10.6) we are then reduced to the analysis of $E_{\mu^{\epsilon}}^{\epsilon}\left(f_{\epsilon}\left(\eta_{t}, \eta_{t+\epsilon^{2 \alpha}}^{(F)}(x) \eta_{t+\epsilon^{2 \alpha}}^{(F)}(y)\right)\right)$ where the process after $t$ is free.

To simplify the computations, it is now convenient to expand $g$ in Poisson polynomials. Referring to Appendix A for definitions and properties, we recall here that the Poisson polynomial of order $n$, denoted by $d_{n}(\cdot)$ and defined in (A.1), is such that $\mathcal{E}_{u}\left(d_{n}\right)=u^{n}$. By (A.3) (and recalling that $g(n) \leqslant \mathrm{e}^{b n}$ ),

$$
g(\eta(x))=\sum_{n=0}^{\infty} \frac{a_{n}}{n !} d_{n}(\eta(x)), \quad\left|a_{n}\right| \leqslant\left|\mathrm{e}^{b}+1\right|^{n} .
$$

For any $\omega \in \mathbb{N}^{\Omega_{\epsilon}}$, let $D_{\omega}(\eta)=\prod_{z \in \Omega_{\epsilon}} d_{\omega(z)}(\eta(z))$, call

$$
\psi_{n}\left(\eta_{t+\epsilon^{2 \alpha}}(z)\right)=d_{n}\left(\eta_{t+\epsilon^{2 \alpha}}(z)\right)-u\left(z, \eta_{t}\right)^{n}
$$

and denote by $E_{\eta_{t}, \xi_{t}}^{\epsilon}$ the conditional expectation given $\eta_{t}, \xi_{t}$ at time $t$. By (A.5)

$$
\begin{aligned}
& E_{\eta_{t}, \xi_{t}}^{\epsilon}\left(\psi_{n}\left(\eta_{t+\epsilon^{2 \alpha}}^{(F)}(x)\right) \psi_{m}\left(\eta_{t+\epsilon^{2 \alpha}}^{(F)}(y)\right)\right) \\
& =\sum_{\omega_{n}^{\prime}, \omega_{m}^{\prime}} \pi_{\epsilon^{-2+2 \alpha}}^{(0)}\left(\omega_{n, x}, \omega_{n}^{\prime}\right) \pi_{\epsilon^{-2+2 \alpha}}^{(0)}\left(\omega_{m, y}, \omega_{m}^{\prime}\right)\left\{D_{\omega_{n}^{\prime}+\omega_{m}^{\prime}}\left(\eta_{t}\right)+u^{\epsilon}\left(x, \eta_{t}\right)^{n} u^{\epsilon}\left(y, \eta_{t}\right)^{m}\right. \\
& \left.\quad-D_{\omega_{n}^{\prime}}\left(\eta_{t}\right) u^{\epsilon}\left(y, \eta_{t}\right)^{m}-D_{\omega_{m}^{\prime}}\left(\eta_{t}\right) u^{\epsilon}\left(x, \eta_{t}\right)^{n}\right\},
\end{aligned}
$$

where $\omega_{k, z}$ is the configuration with $k$ particles at $z$ and $\pi_{t}^{(0)}\left(\omega, \omega^{\prime}\right)$ is the kernel of $\mathrm{e}^{t L^{(\eta, 0)}}$. By (A.10), there is $c(t)$ bounded on the compacts, so that calling $A_{\epsilon, t}(n)=c(t)^{n}\left[1+n ! \epsilon^{n} \mathbf{1}_{n \geqslant \epsilon^{-1}}\right]$,

$$
\begin{aligned}
& \left|E_{\mu^{\epsilon}}^{\epsilon}\left(\mathbf{1}_{u^{\epsilon}\left(x, \eta_{t}\right) \leqslant M, u^{\epsilon}\left(y, \eta_{t}\right) \leqslant M} \psi_{n}\left(\eta_{t+\epsilon^{2 \alpha}}^{(F)}(x)\right) \psi_{m}\left(\eta_{t+\epsilon^{2 \alpha}}^{(F)}(y)\right)\right)\right| \\
& \leqslant A_{\epsilon, t}(n+m)+A_{\epsilon, t}(n) M^{m}+A_{\epsilon, t}(m) M^{n}+M^{n+m} .
\end{aligned}
$$

Thus, recalling (10.7), given any $\delta>0$ there is $N$ so that

$$
\begin{aligned}
& \mid E_{\mu^{\epsilon}}^{\epsilon}\left(f\left(\eta_{t}, \eta_{t+\epsilon^{2 \alpha}}^{(F)}(x) \eta_{t+\epsilon^{2 \alpha}}^{(F)}(y)\right)\right) \\
& \quad-\sum_{n \leqslant N, m \leqslant N} \frac{a_{n} a_{m}}{n ! m !} E_{\mu^{\epsilon}}^{\epsilon}\left(\mathbf{1}_{u^{\epsilon}\left(x, \eta_{t}\right) \leqslant M, u^{\epsilon}\left(y, \eta_{t}\right) \leqslant M} \psi_{n}\left(\eta_{t+\epsilon^{2 \alpha}}^{(F)}(x)\right) \psi_{m}\left(\eta_{t+\epsilon^{2 \alpha}}^{(F)}(y)\right)\right) \mid \leqslant \delta .
\end{aligned}
$$

Theorem 10.2 will then follow from showing that for any fixed $N$, 


$$
\lim _{\epsilon \rightarrow 0} \sup _{x \neq y} \sup _{n, m \leqslant N}\left|E_{\mu^{\epsilon}}^{\epsilon}\left(\mathbf{1}_{u^{\epsilon}\left(x, \eta_{t}\right) \leqslant M, u^{\epsilon}\left(y, \eta_{t}\right) \leqslant M} \psi_{n}\left(\eta_{t+\epsilon^{2 \alpha}}^{(F)}(x)\right) \psi_{m}\left(\eta_{t+\epsilon^{2 \alpha}}^{(F)}(y)\right)\right)\right|=0 .
$$

The proof of (10.9) is reported at the end of Appendix A; it is based on a cancellation on the right-hand side of (10.8). The cancellation involves the terms which appear when $\omega_{n}^{\prime}$ and $\omega_{m}^{\prime}$ are such that $\omega_{n}^{\prime}(x), \omega_{m}^{\prime}(x)$ and $\omega_{n}^{\prime}(x)+\omega_{m}^{\prime}(x)$ are all $\leqslant 1$ for all $x \in \Omega_{\epsilon}$. The other terms involved in the cancellation are the non diagonal terms in the expansions of $u^{\epsilon}\left(z, \eta_{t}\right)^{k}, k=n, m, z=x, y$ (recall the definition (4.2) of $u^{\epsilon}$ as a sum of terms). All the terms mentioned above cancel with each other exactly and, in Appendix A, it is proved that the contribution of all the other terms vanishes proportionally to a positive power of $\epsilon$. The proportionality coefficient has a "bad dependence" on $N$, but since $N$ is held fixed as $\epsilon \rightarrow 0$, this does not give problems.

\section{Homogenization and convergence to the stirring process}

In this section we will prove that the process $\xi_{t}$ is close to the stirring process on sufficiently long time intervals for the latter to reach local equilibrium. By a triangular inequality then $\xi_{t}$ is also locally (close to) Bernoulli, see Theorem 11.1 below. There are essentially two steps in the proof. In the first one we prove a homogenization property at "short times". Recalling that the displacements of the $\xi$ particles are ruled by the generator $\epsilon^{-2 a} L^{(\xi, 0)}$, an effective motion of the $\xi$ particles is only after times of order $\epsilon^{2 a}$. We will see that on the time scale $\epsilon^{2 a}$ the $\xi$ particles are close to stirring with a time dependent intensity determined by the local empirical averages of the $\eta$-particles. The result is then extended to times of order $\epsilon^{2 \beta}$ (recall $\beta<a$ ), thus proving that the $\xi$-particles, like the stirring ones, become (approximately, locally) exchangeable.

The result will be used in Appendix E to control a term $\mathcal{Q}$ which is the most dangerous one among those which appear in the computation of (4.3). After some maquillage operations whose details are given in Appendix E, the space time averages involved in the expression (4.4) lead to study expectations of a measure $\nu_{\tau}^{\epsilon}, \tau>0$, which is a space time average of the original $\mu^{\epsilon} \cdot v_{\tau}^{\epsilon}$ is in fact defined as the probability on $\mathbb{N}^{\Omega_{\epsilon}} \times\{0,1\}^{\Omega_{\epsilon}}$ whose expectations are

$$
\nu_{\tau}^{\epsilon}(f)=\epsilon^{d} \sum_{x \in \Omega_{\epsilon}} f_{0}^{\tau} E_{\mu^{\epsilon}}^{\epsilon}\left(S_{x} f\left(\eta_{t}, \xi_{t}\right)\right), \quad f_{0}^{\tau}=\frac{1}{\tau} \int_{0}^{\tau},
$$

with $S_{x}$ the shift by $x$ on the torus $\Omega_{\epsilon}$.

We fix a large constant $C\left(C=\mathrm{e}^{M}\right.$, see Appendix E), and shorthand

$$
T=C \epsilon^{2 \beta}, \quad B=\left\{x:|x| \leqslant \epsilon^{-a+\beta / 2}\right\} .
$$

We also write $\underline{x}$ for a subset of $\Omega_{\epsilon},|\underline{x}|$ for its cardinality and call

$$
g_{\underline{x}}(\xi)=\prod_{x \in \underline{x}} \xi(x) .
$$

We can now state the main theorem in this section.

Theorem 11.1. Let $T$ as in (11.2), then

$$
\lim _{\epsilon \rightarrow 0} \sup _{\underline{x} \subset B,|\underline{x}|=4}\left|E_{\nu_{\tau}^{\epsilon}}^{\epsilon}\left(g_{\underline{x}}\left(\xi_{T}\right)-\prod_{x \in \underline{x}}\left\{\sum_{z} \pi_{T \epsilon^{-2 a}}(x, z) \xi_{0}(z)\right\}\right)\right|=0 .
$$

We will first prove the theorem for $d \geqslant 3$ and then discuss its extension to $d<3$. The proof is divided in three steps and, from the same proof it will be clear that the result extends to $|\underline{x}| \leqslant n$, for any fixed positive integer $n$.

Step 1: Stirring process and duality. We will prove (11.4) using extensively the self-duality property of the stirring process. Denote by $\underline{x}^{z, z^{\prime}}, z \neq z^{\prime}$ both in $\Omega_{\epsilon}$, the subset of $\Omega_{\epsilon}$ equal to $\underline{x}$ if either $z, z^{\prime}$ are both in $\underline{x}$ or both in $\Omega_{\epsilon} \backslash \underline{x}$. If instead $z \in \underline{x}$ and $z^{\prime} \in \Omega_{\epsilon} \backslash \underline{x}$ then $\underline{x}^{z, z^{\prime}}=z^{\prime} \cup \underline{x} \backslash z$ and if $z \in \Omega_{\epsilon} \backslash \underline{x}$ then $\underline{x}^{z, z^{\prime}}=z \cup \underline{x} \backslash z^{\prime}$. Define

$$
L^{\text {st }} f(\underline{x})=\sum_{z, z^{\prime}:\left|z-z^{\prime}\right|=1 \text { on } \Omega_{\epsilon}}\left[f\left(\underline{x}^{z, z^{\prime}}\right)-f(\underline{x})\right]
$$


the sum in (11.5) being over ordered pairs of nearest neighbor sites on the torus $\Omega_{\epsilon}$ (a bond is counted twice, in agreement with (2.3)). We will also shorthand

$$
p_{t}(\underline{x}, \underline{y})=\mathrm{e}^{t L^{\mathrm{st}}}(\underline{x}, \underline{y}) .
$$

Let $L^{(\xi, \text { st) }}$ be the stirring generator defined in $(2.3)$, then $L^{(\xi, \text { st) }} g_{\underline{x}}(\xi)=L^{\text {st }} g_{\underline{x}}(\xi)$ the former acting on $\xi$, the latter on $\underline{x}$. As a consequence

$$
\mathrm{e}^{t L^{(\xi, \mathrm{st})}} g_{\underline{x}}(\xi)=\sum_{\underline{y}} p_{t}(\underline{x}, \underline{y}) g_{\underline{y}}(\xi) .
$$

To relate (11.4) and (11.7) we should substitute the true process with the stirring one and $p_{t}$ with $\pi_{t}$. The sense in which something like this can be done will become clear in the sequel. Its validity as we will see is limited to suitably short times. The analysis is simpler for $d \geqslant 3$, which is the case we are considering first.

We compare $\epsilon^{-2 a} L^{(\xi, 0)} g_{x}\left(\xi_{t}\right)$ and $\epsilon^{-2 a} c L^{\text {st }} g_{x}\left(\xi_{t}\right)$, the former acting on $\xi_{t}$ the latter on $\underline{x}$, the choice of the intensity $c$ will be crucial. We write

$$
L^{(\xi, 0)} g_{\underline{x}}(\xi)=\sum_{z, z^{\prime}:\left|z-z^{\prime}\right|=1}\left|\xi(z)-\xi\left(z^{\prime}\right)\right|\left(\xi(z) \mathbf{1}_{\eta\left(z^{\prime}\right)=0}+\xi\left(z^{\prime}\right) \mathbf{1}_{\eta(z)=0}\right)\left(g_{\underline{x}}\left(\xi^{z, z^{\prime}}\right)-g_{\underline{x}}(\xi)\right)
$$

and, calling

$$
w^{\epsilon}\left(t, \eta_{0}\right)= \begin{cases}\sum_{y} \pi_{\left(t-\epsilon^{2 \alpha}\right) \epsilon^{-2}(0, y) u^{\epsilon}\left(y, \eta_{0}\right)} & \text { if } t \geqslant \epsilon^{2 \alpha} \\ u^{\epsilon}\left(0, \eta_{0}\right) & \text { otherwise }\end{cases}
$$

we telescopic sum

$$
\begin{aligned}
\mathbf{1}_{\eta_{t}(z)=0}= & {\left[\mathbf{1}_{\eta_{t}(z)=0}-\mathrm{e}^{-u^{\epsilon}\left(z, \eta_{\left.t-\epsilon^{2 \alpha}\right)}\right.}\right]+\left[\mathrm{e}^{-u^{\epsilon}\left(z, \eta_{t-\epsilon^{2 \alpha}}\right)}-\mathrm{e}^{-u^{\epsilon}\left(0, \eta_{t-\epsilon^{2 \alpha}}\right)}\right] } \\
& +\left[\mathrm{e}^{-u^{\epsilon}\left(0, \eta_{t-\epsilon^{2 \alpha}}\right)}-\mathrm{e}^{-w^{\epsilon}\left(t, \eta_{0}\right)}\right]+\mathrm{e}^{-w^{\epsilon}\left(t, \eta_{0}\right)}
\end{aligned}
$$

doing the same for $\mathbf{1}_{\eta_{t}\left(z^{\prime}\right)=0}$. Since $g_{\underline{x}}\left(\xi^{z, z^{\prime}}\right)=g_{\underline{x} z, z^{\prime}}(\xi)$ we get

$$
L^{(\xi, 0)} g_{\underline{x}}\left(\xi_{t}\right)=\mathrm{e}^{-w^{\epsilon}\left(t, \eta_{0}\right)} L^{\mathrm{st}} g_{\underline{x}}\left(\xi_{t}\right)+H_{1}(\underline{x}, t)+\cdots+H_{3}(\underline{x}, t),
$$

where $H_{i}$ comes from the $i$ th square bracket term of (11.8), dependence on $\xi$ and $\eta$ is not explicit here. Since the actual generator of the process is $\epsilon^{-2 a} L^{(\xi, 0)}$, "the error terms" $H_{i}$ will be eventually multiplied by $\epsilon^{-2 a}$.

Step 2: Reduction to stirring. (11.9) is the desired duality relation which establishes that modulo "the errors" $H_{i}$ we can compute the expectation of $g_{\underline{x}}\left(\xi_{t}\right)$ by applying the stirring to $g_{\underline{x}}$ thought of as a function of $\underline{x}$. Define the "total intensity"

$$
A\left(\eta_{0}\right):=\int_{0}^{T} \mathrm{e}^{-w^{\epsilon}\left(t, \eta_{0}\right)}, \quad T=C \epsilon^{2 \beta},
$$

and recall that in the whole sequel, $\underline{x}^{0} \subset B$ and $\left|\underline{x}^{0}\right|=4$ (we rename by $\underline{x}^{0}$ the set $x$ in (11.2) where $B$ is also defined). We set $2 B=\left\{x:|x| \leqslant 2 \epsilon^{-a+\beta / 2}\right\}$. Using (11.9) and recalling (11.6), we get

$$
\begin{aligned}
& E_{\nu_{\tau}^{\epsilon}}^{\epsilon}\left(g_{\underline{x}^{0}}\left(\xi_{T}\right)\right)=E_{\nu_{\tau}^{\epsilon}}^{\epsilon}\left(\sum_{\underline{y}} p_{A\left(\eta_{0}\right) \epsilon^{-2 a}}\left(\underline{x}^{0}, \underline{y}\right) g_{\underline{y}}\left(\xi_{0}\right)\right)+\mathcal{R}, \\
& \mathcal{R}=\int_{0}^{T} \sum_{\underline{y}} p_{(T-s) \epsilon^{-2 a}}\left(\underline{x}^{0}, \underline{y}\right) E_{v_{\tau}^{\epsilon}}^{\epsilon}\left(\left\{L^{\prime} g_{\underline{y}}\left(\xi_{s}\right)+\epsilon^{-2 a}\left[H_{1}(\underline{y}, s)+H_{2}(\underline{y}, s)+H_{3}(\underline{y}, s)\right]\right\}\right),
\end{aligned}
$$

where $L^{\prime}=L^{(\xi,+)}+L^{(\xi,-)}$.

We will next prove that there is $c>0$ so that

$$
|\mathcal{R}| \leqslant c \epsilon^{2 \beta-2 a+2 \beta} \text {. }
$$


The contribution of the times $t \leqslant \epsilon^{2 \alpha}$ is bounded by $c \epsilon^{-2 a} \epsilon^{2 \alpha}$ and since $\alpha>2 \beta$, this is compatible with (11.11). Let us now consider $t \geqslant \epsilon^{2 \alpha}$.

Since $\left|\mathrm{e}^{-u^{\epsilon}\left(0, \eta_{t-\epsilon^{2 \alpha}}\right)}-\mathrm{e}^{-w^{\epsilon}\left(t, \eta_{0}\right)}\right| \leqslant c\left|u^{\epsilon}\left(0, \eta_{t-\epsilon^{2 \alpha}}\right)-w^{\epsilon}\left(t, \eta_{0}\right)\right|$, by using Theorem 9.1 the term containing $H_{3}$ ( $H_{3}$ is the third square bracket on the right-hand side of (11.8)) is bounded by $c \epsilon^{2 \beta-2 a} T$. All the other terms are smaller. In fact, by (10.1) the term with $H_{1}$ is bounded proportionally to $\epsilon^{2 \beta-2 a+2 \alpha}$, so that, by the choice of $\alpha$, it is $\leqslant c \epsilon^{2 \beta-2 a+2 \beta}$.

We decompose the sum over $y$ in the term with $H_{2}$ as over $y \subset 2 B$ and the complement:

$$
\sum_{\underline{y}} p_{(T-s) \epsilon^{-2 a}}\left(\underline{x}^{0}, \underline{y}\right) H_{2}(\underline{y}, s)=\sum_{\underline{y} \subset 2 B} p_{(T-s) \epsilon^{-2 a}}\left(\underline{x}^{0}, \underline{y}\right) H_{2}(\underline{y}, s)+\sum_{\underline{y} \not \subset 2 B} p_{(T-s) \epsilon^{-2 a}}\left(\underline{x}^{0}, \underline{y}\right) H_{2}(\underline{y}, s) .
$$

The first term is bounded using (8.18) and (8.15), proportionally to $\epsilon^{2 \beta-2 a+1-a+\beta / 2}$. The second term is bounded by the probability that there is at least a particle which travels by $\epsilon^{-a+\beta / 2}$ in a time $\epsilon^{-2 a+2 \beta}$ and it is therefore bounded by $c \exp \left\{-c^{\prime} \epsilon^{-\beta}\right\}$. Finally, the term with $L^{\prime} g_{\underline{y}}\left(\xi_{s}\right)$ is bounded by $c \epsilon^{2 \beta}$, because, by Theorem $8.1, E_{\nu_{\tau}^{\epsilon}}^{\epsilon}\left(\kappa_{\eta_{s}(\cdot)+1}\right) \leqslant c$.

The bound in (11.11) vanishes as $\epsilon \rightarrow 0$ if $2 \beta>a$. Since $\beta$ must satisfy the inequality $\beta<\frac{d}{d+2} a$ (see for instance the proof of Theorem 8.3), we must then have

$$
\frac{d}{d+2} a>\frac{a}{2}
$$

hence the restriction to $d \geqslant 3$.

Step 3: Conclusion. By (11.10) and (11.11),

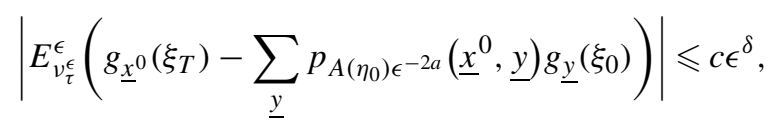

where $c \epsilon^{\delta}, \delta>0$, shorthands the bound (11.11) on the remainders and

$$
\epsilon^{-2 a} A\left(\eta_{0}\right)=\epsilon^{-2 a} \int_{0}^{C \epsilon^{2 \beta}} \mathrm{e}^{-w^{\epsilon}\left(t, \eta_{0}\right)}
$$

with $w^{\epsilon}\left(t, \eta_{0}\right)=\sum_{y} \pi_{\left(t-\epsilon^{2 \alpha}\right) \epsilon^{-2}}(0, y) u^{\epsilon}\left(y, \eta_{0}\right)$ for $t \geqslant \epsilon^{2 \alpha}$ and $w^{\epsilon}\left(t, \eta_{0}\right)=u^{\epsilon}\left(0, \eta_{0}\right)$ for $t<\epsilon^{2 \alpha}$. We claim that the following two inequalities hold:

$$
\begin{aligned}
& \nu_{\tau}^{\epsilon}\left(\epsilon^{-2 a} A\left(\eta_{0}\right) \leqslant C \mathrm{e}^{-M} \epsilon^{-2 a+2 \beta}\right) \leqslant c \epsilon^{\beta}, \\
& E_{\nu_{\tau}^{\epsilon}}\left(f_{0}^{C \epsilon^{2 \beta}}\left|\mathrm{e}^{-w^{\epsilon}\left(t, \eta_{0}\right)}-\mathrm{e}^{-u^{\epsilon}\left(0, \eta_{0}\right)}\right|\right) \leqslant c \epsilon^{\beta} .
\end{aligned}
$$

Proof of (11.14), (11.15). By (8.2),

$$
\nu_{\tau}^{\epsilon}\left(\mathrm{e}^{-u^{\epsilon}(0, \eta)} \leqslant \mathrm{e}^{-M} / 2\right) \leqslant c \epsilon^{(1-\alpha) d}
$$

so that

$$
\begin{aligned}
\text { 1.h.s. of }(11.14) & =v_{\tau}^{\epsilon}\left(\int_{0}^{C \epsilon^{2 \beta}} \mathrm{e}^{-w^{\epsilon}\left(t, \eta_{0}\right)}<\mathrm{e}^{-M}\right) \\
& \leqslant c \epsilon^{(1-\alpha) d}+v_{\tau}^{\epsilon}\left(\int_{0}^{C \epsilon^{2 \beta}}\left|\mathrm{e}^{-w^{\epsilon}\left(t, \eta_{0}\right)}-\mathrm{e}^{-u^{\epsilon}\left(0, \eta_{0}\right)}\right| \geqslant \frac{\mathrm{e}^{-M}}{2}\right)
\end{aligned}
$$

and, since $(1-\alpha) d>\beta$, (11.14) follows from (11.15), which we prove next: 


$$
\begin{aligned}
v_{\tau}^{\epsilon}\left(\int_{0}^{C \epsilon^{2 \beta}}\left|\mathrm{e}^{-w^{\epsilon}\left(t, \eta_{0}\right)}-\mathrm{e}^{-u^{\epsilon}\left(0, \eta_{0}\right)}\right|\right) & \leqslant \int_{0}^{C \epsilon^{2 \beta}} v_{\tau}^{\epsilon}\left(\sum_{y} \pi_{\left(t-\epsilon^{2 \alpha}\right) \epsilon^{-2}}(0, y)\left|u^{\epsilon}\left(y, \eta_{0}\right)-u^{\epsilon}\left(0, \eta_{0}\right)\right|\right) \\
& \leqslant \epsilon \sqrt{d}\left\{\int_{0}^{C \epsilon^{2 \beta}} \sum_{y} \pi_{\left(t-\epsilon^{2 \alpha}\right) \epsilon^{-2}}(0, y)|y|\right\} v_{\tau}^{\epsilon}\left(\left\|u^{\epsilon}\left(\eta_{0}\right)\right\|_{H_{1}^{2}}\right)
\end{aligned}
$$

having used (8.16). Since $\sum_{y} \pi_{\left(t-\epsilon^{2 \alpha}\right) \epsilon^{-2}}(0, y)|y| \leqslant c \epsilon^{-1}\left(t-\epsilon^{2 \alpha}\right)^{1 / 2} \leqslant c \epsilon^{-1+\beta}$ and

$$
\nu_{\tau}^{\epsilon}\left(\left\|u^{\epsilon}\left(\eta_{s}\right)\right\|_{H_{1}^{2}}\right)=\int_{0}^{\tau} E_{\mu^{\epsilon}}^{\epsilon}\left(\left\|u^{\epsilon}\left(\eta_{t}\right)\right\|_{H_{1}^{2}}\right) \leqslant c \tau^{-1 / 2}
$$

(the last inequality follows from (8.9) and Cauchy-Schwartz), (11.15) and hence (11.14), (11.15) are proved.

Using (11.13), (11.14) and (B.2) (recall that $A\left(\eta_{0}\right) \leqslant C \epsilon^{2 \beta}$ ), we have

$$
\left|E_{\nu_{\tau}^{\epsilon}}^{\epsilon}\left(g_{\underline{x}^{0}}\left(\xi_{T}\right)-\left\{\prod_{x \in \underline{x}} \sum_{y} \pi_{A\left(\eta_{0}\right) \epsilon^{-2 a}}(x, y)\right\} g_{\underline{y}}\left(\xi_{0}\right)\right)\right| \leqslant c\left[\epsilon^{\delta}+\epsilon^{\beta}+\epsilon^{(-2 a+2 \beta) / 12}\right] .
$$

We are going to use the following classical estimate on random walks,

$$
\sum_{y}\left|\pi_{t^{\prime}}(x, y)-\pi_{t}(x, y)\right| \leqslant c \frac{\left|t-t^{\prime}\right|}{\min \left\{t, t^{\prime}\right\}} .
$$

$c$ a suitable constant. We take $t=A\left(\eta_{0}\right) \epsilon^{-2 a}$ and $t^{\prime}=C \epsilon^{-2 a+2 \beta} \mathrm{e}^{-u^{\epsilon}(0, \eta)}$. Then, by (11.15),

$$
\left|t-t^{\prime}\right| \leqslant \epsilon^{\beta / 2}\left[C \epsilon^{-2 a+2 \beta}\right] \text { with probability } \geqslant 1-c \epsilon^{\beta / 2}
$$

and by (11.14)

$$
\min \left\{t, t^{\prime}\right\} \geqslant C \mathrm{e}^{-M} \epsilon^{-2 a+2 \beta}-\epsilon^{\beta / 2}\left[C \epsilon^{-2 a+2 \beta}\right] \text { with probability } \geqslant 1-c \epsilon^{\beta / 2}-c \epsilon^{\beta} .
$$

Then

$$
E_{v_{\tau}^{\epsilon}}\left(\sum_{y}\left|\pi_{A\left(\eta_{0}\right) \epsilon^{-2 a}}(x, y)-\pi_{C \epsilon^{-2 a+2 \beta} \mathrm{e}^{-\epsilon^{\epsilon}\left(0, \eta_{0}\right)}}(x, y)\right|\right) \leqslant \frac{C \epsilon^{-2 a+2 \beta+\beta / 2}}{\left(C \mathrm{e}^{-M} / 2\right) \epsilon^{-2 a+2 \beta}}+c \epsilon^{\beta / 2}
$$

thus completing the proof of the theorem for $d \geqslant 3$.

The cases $d=1,2$. In $d=1,2$ the bound in (11.11) does not vanish and the idea is to apply the previous analysis for a time shorter than $\epsilon^{2 \beta}$, say $\epsilon^{2 \gamma}, \gamma>\beta$. The error is then bounded as in (11.11) but with $\epsilon^{2 \gamma-2 a+2 \gamma}$. If an iterative procedure applied we would repeat such an error $\epsilon^{2 \beta-2 \gamma}$ times, thus we need $\epsilon^{2 \gamma-2 a+2 \gamma} \epsilon^{2 \beta-2 \gamma}$ to vanish, namely $\gamma+\beta>a$. Let $\vartheta>0$ be so small that $\frac{d-\vartheta}{d+2}>\frac{5}{17}$ (the last inequality will be needed at the end, to have (11.22) vanishing as $\epsilon \rightarrow 0$ ) and set for $d=1,2$,

$$
\gamma=\frac{2+2 \vartheta}{d+2} a, \quad \beta=\frac{d-\vartheta}{d+2} a
$$

so that, with such a choice,

$$
a>\gamma>a-\beta, \quad \frac{d}{d+2} a>\beta>\frac{5 a}{17} .
$$

We divide the time interval $[0, T]$ into intervals of lengths $\epsilon^{2 \gamma}$ except maybe the last one which has length $\leqslant \epsilon^{2 \gamma}$. The intervals are called $\left[t_{n}, t_{n+1}\right), n \leqslant N$, and the last one is $\left[t_{N}, t_{N+1}\right], t_{N+1}=T$. Instead of $w^{\epsilon}\left(t, \eta_{0}\right)$ we now consider the quantities

$$
w^{\epsilon}\left(t, t_{n-1}, \eta_{t_{n-1}}\right)=\sum_{y} \pi_{\left(t-\epsilon^{2 \alpha}-t_{n-1}\right) \epsilon^{-2}}(0, y) u^{\epsilon}\left(y, \eta_{t_{n-1}}\right), \quad t \in\left(t_{n}, t_{n+1}\right] .
$$

Analogously to (11.8) we write 


$$
\begin{aligned}
\mathbf{1}_{\eta_{t}(z)=0}= & {\left[\mathbf{1}_{\eta_{t}(z)=0}-\mathrm{e}^{-u^{\epsilon}\left(z, \eta_{\left.t-\epsilon^{2 \alpha}\right)}\right.}\right]+\left[\mathrm{e}^{-u^{\epsilon}\left(z, \eta_{t-\epsilon^{2 \alpha}}\right)}-\mathrm{e}^{-u^{\epsilon}\left(0, \eta_{t-\epsilon^{2 \alpha}}\right)}\right] } \\
& +\left[\mathrm{e}^{-u^{\epsilon}\left(0, \eta_{t-\epsilon^{2 \alpha}}\right)}-\mathrm{e}^{-w^{\epsilon}\left(t, t_{n-1}, \eta_{t_{n-1}}\right)}\right]+\mathrm{e}^{-w^{\epsilon}\left(t, t_{n-1}, \eta_{t_{n-1}}\right)}
\end{aligned}
$$

and get instead of (11.9),

$$
L^{(\xi, 0)} g_{\underline{x}}\left(\xi_{t}\right)=\mathrm{e}^{-w^{\epsilon}\left(t, t_{n-1}, \eta_{t_{n}}\right)} L^{\mathrm{st}} g_{\underline{x}}\left(\xi_{t}\right)+H_{1}(\underline{x}, t)+\cdots+H_{3}(\underline{x}, t) .
$$

$A\left(\eta_{0}\right)$ is now replaced by

$$
A_{t_{n}, t_{j}}\left(\eta_{t_{j}}\right):=\int_{t_{n}}^{t_{n+1}} \mathrm{e}^{-w^{\epsilon}\left(t, t_{j}, \eta_{t_{j}}\right)}, \quad j<n,
$$

and, analogously to (11.10) we have

$$
\begin{aligned}
& E_{v_{\tau}^{\epsilon}}^{\epsilon}\left(g_{\underline{x}^{0}}\left(\xi_{T}\right)\right)=E_{v_{\tau}^{\epsilon}}^{\epsilon}\left(\sum_{\underline{y}} p_{A_{t_{N}, t_{N-1}}\left(\eta_{t_{N-1}}\right) \epsilon^{-2 a}}\left(\underline{x}^{0}, \underline{y}\right) g_{\underline{y}}\left(\xi_{t_{N}}\right)\right)+\mathcal{R}_{N}^{(1)}, \\
& \mathcal{R}_{n}^{(1)}=\int_{t_{n}}^{t_{n+1}} \sum_{\underline{y}} p_{\left(t_{N}-t\right) \epsilon^{-2 a}}\left(\underline{x}^{0}, \underline{y}\right) E_{\nu_{\tau}^{\epsilon}}^{\epsilon}\left(\left\{L^{\prime} g_{\underline{y}}\left(\xi_{s}\right)+\epsilon^{-2 a}\left[H_{1}(\underline{y}, s)+\cdots+H_{3}(\underline{y}, s)\right]\right\}\right) .
\end{aligned}
$$

We are not yet ready for the iteration, because the first term on the right-hand side of (11.17) has not the same expression as the term we started from, due to its dependence on $\eta_{t_{N-1}}$. To fix the problem we do a Taylor-Lagrange expansion to first order in the small parameter $\epsilon^{-2 a}\left[A_{t_{N}, t_{N-1}}\left(\eta_{t_{N-1}}\right)-A_{t_{N}, 0}\left(\eta_{0}\right)\right]$ : indeed, using Theorem 9.1,

$$
\epsilon^{-2 a}\left[A_{t_{N}, t_{N-1}}\left(\eta_{t_{N-1}}\right)-A_{t_{N}, 0}\left(\eta_{0}\right)\right] \leqslant c \epsilon^{-2 a+2 \gamma+2 \beta} .
$$

Shorthand

$$
b_{n}(\lambda):=\lambda A_{t_{n}, t_{n-1}}\left(\eta_{t_{n-1}}\right)+(1-\lambda) A_{t_{n}, 0}\left(\eta_{0}\right)
$$

then

$$
E_{\nu_{\tau}^{\epsilon}}^{\epsilon}\left(g_{\underline{x}^{0}}\left(\xi_{t_{N+1}}\right)\right)=\sum_{\underline{y}} p_{A_{t_{N}, 0}\left(\eta_{0}\right) \epsilon^{-2 a}}\left(\underline{x}^{0}, \underline{y}\right) E_{v_{\tau}^{\epsilon}}^{\epsilon}\left(g_{\underline{y}}\left(\xi_{t_{N}}\right)\right)+\mathcal{R}_{N}^{(1)}+\mathcal{R}_{N}^{(2)},
$$

where

$$
\mathcal{R}_{N}^{(2)}=\int_{0}^{1} E_{\nu_{\tau}^{\epsilon}}^{\epsilon}\left(\sum_{\underline{y}}\left[A_{t_{N}, t_{N-1}}\left(\eta_{t_{N-1}}\right)-A_{t_{N}, 0}\left(\eta_{0}\right)\right] \epsilon^{-2 a} p_{b_{N}(\lambda) \epsilon^{-2 a}}\left(\underline{x}^{0}, \underline{y}\right) L^{\mathrm{st}} g_{\underline{y}}\left(\xi_{t_{N}}\right)\right) \mathrm{d} \lambda .
$$

The first term on the right-hand side of (11.18) has now the same structure as its left-hand side so we can iterate. Calling

$$
A^{(n)}\left(\eta_{0}\right):=A_{t_{N}, 0}\left(\eta_{0}\right)+\cdots+A_{t_{n}, 0}\left(\eta_{0}\right), \quad n \leqslant N, \quad A^{(N+1)}\left(\eta_{0}\right) \equiv 0, \quad A^{(0)}\left(\eta_{0}\right)=A\left(\eta_{0}\right)
$$

we get

$$
E_{\nu_{\tau}^{\epsilon}}^{\epsilon}\left(g_{\underline{x}^{0}}\left(\xi_{t_{N+1}}\right)\right)=E_{v_{\tau}^{\epsilon}}^{\epsilon}\left(\sum_{\underline{y}} p_{A^{(0)}\left(\eta_{0}\right) \epsilon^{-2 a}}\left(\underline{x}^{0}, \underline{y}\right) g_{\underline{y}}\left(\xi_{0}\right)\right)+\sum_{i=1}^{2} \sum_{n=0}^{N} \mathcal{R}_{n}^{(i)},
$$

where, analogously to (11.19),

$$
\mathcal{R}_{n}^{(2)}=\int_{0}^{1} E_{v_{\tau}^{\epsilon}}^{\epsilon}\left(\sum_{\underline{y}}\left[A_{t_{n}, t_{n-1}}\left(\eta_{t_{n-1}}\right)-A_{t_{n}, 0}\left(\eta_{0}\right)\right] \epsilon^{-2 a} p_{\left[A^{(n+1)}\left(\eta_{0}\right)+b_{n}(\lambda)\right] \epsilon^{-2 a}}\left(\underline{x}^{0}, \underline{y}\right) L^{\mathrm{st}} g_{\underline{y}}\left(\xi_{t_{n}}\right)\right) \mathrm{d} \lambda .
$$


By the same arguments used in $d \geqslant 3$, the contribution of $\mathcal{R}_{n}^{(1)}$ is bounded by $c \epsilon^{2 \gamma-2 a+2 \gamma}$. The contribution of $\mathcal{R}_{n}^{(2)}$ is bounded by

$$
c \epsilon^{2 \gamma-2 a+2 \beta}\left[\left(t_{N}-t_{n}\right) \epsilon^{-2 a}\right]^{-1 / 2-1 / 12}
$$

$c$ a positive constant. The bound is derived in Appendix B by exploiting estimates on the stirring process known in the literature.

The total contribution of all the remainders is then bounded by

$$
c\left\{\epsilon^{2 \gamma-2 a+2 \gamma} \epsilon^{2 \beta-2 \gamma}+\epsilon^{-2 a+2 \beta} T^{1 / 2-1 / 12} \epsilon^{2 a(1 / 2+1 / 12)}\right\}
$$

with $T=C \epsilon^{2 \beta}$. The first term vanishes by (11.16), the second one because $\beta>5 a / 17$, see again (11.16). We have thus proved (11.13) and since the rest of the proof is like in the last step of the case $d \geqslant 3$, Theorem 11.1 is proved.

\section{Appendix A. Independent and branching random walks}

We will prove here the statements in the text which refer to independent random walks without and with independent branchings. We start by recalling the main properties of the former.

Poisson polynomials. The Poisson polynomials $d_{k}(\cdot)$ of degree $k \geqslant 0$ are

$$
d_{0}(n) \equiv 1, \quad d_{1}(n)=n, \quad d_{k}(n)=n(n-1) \cdots(n-k+1), \quad k>1,
$$

and we restrict $n \in \mathbb{N}$. The following remarkable identities hold for any non negative integer $\eta(x)$,

$$
\begin{aligned}
& \mathbf{1}_{\eta(x)=i}=\sum_{n=0}^{\infty} \frac{(-1)^{n}}{n !} d_{n+i}(\eta(x)), \\
& \mathrm{e}^{b \eta(x)}=\sum_{n=0}^{\infty} \frac{\left(\mathrm{e}^{b}-1\right)^{n}}{n !} d_{n}(\eta(x)) .
\end{aligned}
$$

If $f(n)$ is exponentially bounded, say $|f(n)| \leqslant \mathrm{e}^{b n}$, then

$$
f(\eta(x))=\sum_{n=0}^{\infty} \frac{a_{n}}{n !} d_{n}(\eta(x)), \quad\left|a_{n}\right| \leqslant\left|\mathrm{e}^{b}+1\right|^{n} .
$$

The above will be applied to $\kappa_{\eta(x)}$ (and to $\kappa_{\eta(x)}^{n}, n \in \mathbb{N}$, as well), recalling that $\kappa_{\eta(x)}$, which is the function which appears in the generator $L$ in Section 2, is supposed to be exponentially bounded.

Poisson multi-polynomials. The Poisson multi-polynomial $D_{\omega}, \omega: \Omega_{\epsilon} \rightarrow \mathbb{N}$, is

$$
D_{\omega}(\eta)=\prod_{x \in \Omega_{\epsilon}} d_{\omega(x)}(\eta(x)) .
$$

Duality. Referring to [4] for details, the self-duality property of the independent process is expressed by the equality

$$
L^{(\eta, 0)} D_{\omega}(\eta)=L^{(\omega, 0)} D_{\omega}(\eta) .
$$

Namely the independent generator gives the same result when applied to $D_{\omega}(\eta)$ both if it acts on $\omega$ or on $\eta$. Denoting by

$$
\pi_{t}^{(0)}(\cdot, \cdot)=\mathrm{e}^{t L^{(\cdot, 0)}}(\cdot, \cdot)
$$

the kernel of $\mathrm{e}^{t L^{(\cdot, 0)}}$, it then follows from (A.4) that

$$
\sum_{\eta^{\prime}} \pi_{t}^{(0)}\left(\eta, \eta^{\prime}\right) D_{\omega}\left(\eta^{\prime}\right)=\sum_{\omega^{\prime}} \pi_{t}^{(0)}\left(\omega, \omega^{\prime}\right) D_{\omega^{\prime}}(\eta) .
$$

Using (A.5) in connection with the integration by parts formula, we get 


$$
\mathrm{e}^{\left(L^{(\eta, 0)}+L^{\prime}\right) t} D_{\omega}=\sum_{\omega^{\prime}} \pi_{t}^{(0)}\left(\omega, \omega^{\prime}\right) D_{\omega^{\prime}}+\int_{0}^{t} \sum_{\omega^{\prime}} \pi_{t-s}^{(0)}\left(\omega, \omega^{\prime}\right) \mathrm{e}^{\left(L^{(\eta, 0)}+L^{\prime}\right) s}\left\{L^{\prime} D_{\omega^{\prime}}\right\}
$$

where $L^{\prime}$ is a generator acting on functions of $(\eta, \xi)$.

Notation. We will use the following notation: $1_{x}$ stands for the configuration $\omega$ with only one particle at $x ; \omega \pm$ $\omega^{\prime}$ for the configuration with $\omega(x) \pm \omega^{\prime}(x)$ at $x$ (the relation with the minus sign being defined only if $\omega^{\prime} \leqslant \omega$ ); $|\omega|=\sum_{x \in \Omega_{\epsilon}} \omega(x)$. Then

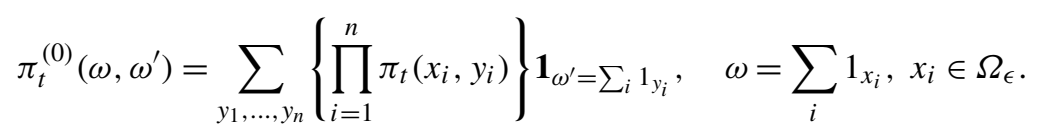

In particular $\pi_{t}^{(0)}=\pi_{t}$ when restricted to $\omega:|\omega|=1$.

Proof of Theorem 5.1. We will prove here only the statements of the theorem relative to the variables $\eta$. Recalling (5.2), by (A.5),

$$
U_{0}^{\epsilon}(x)=\mu^{\epsilon}\left(u^{\epsilon}(x, \eta)\right)=\sum_{y} p_{\alpha}(x, y) \mu^{\epsilon}(\eta(y))=\sum_{y} p_{\alpha}(x, y) \sum_{z} \pi_{\epsilon^{-2} t_{0}}(y, z) \eta_{-t_{0}}^{\epsilon}(z) .
$$

Thus $\left|U_{0}^{\epsilon}(x)\right| \leqslant C$, if $C$ is the sup in (5.1). Moreover,

$$
\epsilon^{-1}\left|\nabla U_{0}^{\epsilon}(x)\right| \leqslant \epsilon^{-1} \sum_{z}\left|\nabla \pi_{\epsilon^{-2} t_{0}}(0, z)\right| \leqslant \frac{c}{\sqrt{t_{0}}}
$$

which proves the first inequality in (5.5).

For future reference we also observe that the same argument used above shows that

$$
\mu^{\epsilon}\left(D_{\omega}(\eta)\right)=\sum_{\omega^{\prime}} \pi_{\epsilon^{-2} t_{0}}^{(0)}\left(\omega, \omega^{\prime}\right) D_{\omega^{\prime}}\left(\eta_{-t_{0}}\right) \leqslant C^{|\omega|} .
$$

Call $\tilde{\eta}(x)=\eta(x)-\mu^{\epsilon}(\eta(x))$, then

$$
\mu^{\epsilon}\left(\left\|u^{\epsilon}-U^{\epsilon}\right\|^{2}\right)=\epsilon^{d} \sum_{x \in \Omega_{\epsilon}} \sum_{z, z^{\prime}} p_{\alpha}(x, z) p_{\alpha}\left(x, z^{\prime}\right) \mu^{\epsilon}\left(\tilde{\eta}(z) \tilde{\eta}\left(z^{\prime}\right)\right) .
$$

Shorthanding $\omega_{z}=1_{z}$ and $\omega_{z, z^{\prime}}=1_{z}+1_{z^{\prime}}$,

$$
\mu^{\epsilon}\left(\tilde{\eta}(z) \tilde{\eta}\left(z^{\prime}\right)\right)=\mu^{\epsilon}\left(D_{\omega_{z, z^{\prime}}}\right)-\mu^{\epsilon}\left(D_{\omega_{z}}\right) \mu^{\epsilon}\left(D_{\omega_{z^{\prime}}}\right)+\mathbf{1}_{z=z^{\prime}} \mu^{\epsilon}(\eta(z))
$$

so that

$$
\mu^{\epsilon}\left(\left\|u^{\epsilon}-U^{\epsilon}\right\|^{2}\right)=\epsilon^{d} \sum_{x \in \Omega_{\epsilon}}\left\{\sum_{z \in \Omega_{\epsilon}} p_{\alpha}(x, z)^{2} \mu^{\epsilon}(\eta(z))-\sum_{z \in \Omega_{\epsilon}}\left[\sum_{y \in \Omega_{\epsilon}} p_{\alpha}(x, y) \pi_{\epsilon^{-2} t_{0}}(y, z)\right]^{2} \eta_{-t_{0}}(z)\right\} \leqslant c \epsilon^{(1-\alpha) d} .
$$

The last inequality follows using the local central limit theorem to bound

$$
\sup _{z \in \Omega_{\epsilon}} p_{\alpha}(0, z) \leqslant \frac{c}{\epsilon^{-(2-2 \alpha) d / 2}}, \quad \sup _{z \in \Omega_{\epsilon}}\left[\sum_{y \in \Omega_{\epsilon}} p_{\alpha}(x, y) \pi_{\epsilon^{-2} t_{0}}(y, z)\right] \leqslant \frac{c}{\left[\epsilon^{-(2-2 \alpha)}+\epsilon^{-2} t_{0}\right]^{d / 2}} .
$$

The statements in Theorem 5.1 relative to the $\eta$ variables are proved.

The next theorem will be used in the proof of Theorem 8.1. We recall for the reader's convenience the Stirling formula

$$
n !=n^{n+1 / 2} \mathrm{e}^{-n} \sqrt{2 \pi}\left(1+\mathrm{O}\left(\frac{1}{\sqrt{n}}\right)\right)
$$

observing that the last bracket in (A.10) below is then bounded by 1 (for $\epsilon$ small enough). Then (A.10) extends the bound (A.8) to all times, provided the constant $C$ is replaced by $\mathrm{e}^{\kappa t}(C+2 c \sqrt{t})$. 
Theorem A.1. There is a constant $c>0$ so that for any $t>0$ and any $\omega$,

$$
E_{\mu^{\epsilon}}^{\epsilon}\left(D_{\omega}\left(\eta_{t}\right)\right) \leqslant E_{\mu^{\epsilon}}^{\epsilon}\left(D_{\omega}\left(\eta_{t}^{+}\right)\right) \leqslant\left(\mathrm{e}^{\kappa t}(1+2 t)(C+2 c \sqrt{t})\right)^{|\omega|}\left(1_{|\omega| \leqslant \epsilon^{-1}}+|\omega| !(\mathrm{e} \epsilon)^{|\omega|} 1_{|\omega| \geqslant \epsilon^{-1}}\right),
$$

where $\eta_{t}^{+}$is the process with generator $\epsilon^{-2} L^{(\eta, 0)}+L^{(\eta,+)}$.

Proof. We will first prove that:

$$
L^{(\eta,+)} D_{\omega}(\eta)=\kappa|\omega| D_{\omega}(\eta)+\kappa \sum_{x \in \Omega_{\epsilon}} \omega(x)[\omega(x)-1] D_{\omega-1_{x}}(\eta)
$$

(recall that $|\omega|=\sum_{x} \omega(x),\left[\omega-1_{x}\right](z)=\omega(z)$ for all $z \neq x$ and $\left.\left[\omega-1_{x}\right](x)=\omega(x)-1\right)$. Indeed

$$
L^{(\eta,+)} D_{\omega}(\eta)=\kappa \sum_{x}\left[\prod_{y \neq x} d_{\omega(y)}(\eta(y))\right] \eta(x)\left[d_{\omega(x)}(\eta(x)+1)-d_{\omega(x)}(\eta(x))\right]
$$

and (A.11) follows because $D_{\omega}(\eta)=0$ unless $\eta \geqslant \omega$ and because for $k \geqslant 1$,

$$
k\left[d_{n}(k+1)-d_{n}(k)\right]=n d_{n}(k)+n[n-1] d_{n-1}(k) .
$$

By (A.6) and (A.11),

$$
\begin{aligned}
E_{\mu^{\epsilon}}^{\epsilon}\left(D_{\omega}\left(\eta_{t}^{+}\right)\right)= & \sum_{\omega^{\prime}} \pi_{t}^{(0)}\left(\omega, \omega^{\prime}\right) \mu^{\epsilon}\left(D_{\omega^{\prime}}(\eta)\right)+\kappa|\omega| \int_{0}^{t} \sum_{\omega^{\prime}} \pi_{t-s}^{(0)}\left(\omega, \omega^{\prime}\right) E_{\mu^{\epsilon}}^{\epsilon}\left(D_{\omega^{\prime}}\left(\eta_{s}^{+}\right)\right) \\
& +\kappa \sum_{y \in \Omega_{\epsilon}} \int_{0}^{t} \sum_{\omega^{\prime}} \pi_{t-s}^{(0)}\left(\omega, \omega^{\prime}\right) \omega^{\prime}(y)\left[\omega^{\prime}(y)-1\right] E_{\mu^{\epsilon}}^{\epsilon}\left(D_{\omega^{\prime}-1_{y}}\left(\eta_{s}^{+}\right)\right) .
\end{aligned}
$$

We postpone to the end of the proof that

$$
\text { last term in }(\mathrm{A} .12) \leqslant c \epsilon n(n-1) \int_{0}^{t} \frac{1}{\sqrt{t-s}} \sup _{\omega^{*}:\left|\omega^{*}\right|=n-1} \sum_{\omega^{\prime}:\left|\omega^{\prime}\right|=n-1} \pi_{t-s}^{(0)}\left(\omega^{*}, \omega^{\prime}\right) E_{\mu^{\epsilon}}^{\epsilon}\left(D_{\omega^{\prime}}\left(\eta_{s}^{+}\right)\right) .
$$

Call $v_{k}(s)=\max _{|\omega|=k} E_{\mu^{\epsilon}}^{\epsilon}\left(D_{\omega}\left(\eta_{s}^{+}\right)\right)$. Since $\mu^{\epsilon}\left(D_{\omega}\left(\eta_{0}\right)\right) \leqslant C^{|\omega|}$, by (A.12) and (A.13)

$$
\begin{aligned}
v_{n}(t) & \leqslant \mathrm{e}^{\kappa n t} C^{n}+c \in n(n-1) \int_{0}^{t}\left\{\frac{1}{\sqrt{t-s}}+\int_{s}^{t} \frac{\mathrm{e}^{\kappa n\left(t-s^{\prime}\right)}}{\sqrt{s^{\prime}-s}}\right\} v_{n-1}(s) \\
& \leqslant \mathrm{e}^{\kappa n t} C^{n}+c \in n(n-1)(1+2 t) \int_{0}^{t} \frac{\mathrm{e}^{\kappa n(t-s)}}{\sqrt{t-s}} v_{n-1}(s)
\end{aligned}
$$

and, by iteration,

$$
v_{n}(t) \leqslant \mathrm{e}^{\kappa n t}\left(C^{n}+\sum_{k=1}^{n-1}[c \epsilon(1+2 t)]^{k}\left[n(n-1)^{2} \cdots(n-k+1)^{2}(n-k)\right] I_{k}(t) C^{n-k}\right),
$$

where

$$
I_{k}(t)=\int_{0}^{t} \frac{1}{\sqrt{t-s_{1}}} \cdots \int_{0}^{s_{k-1}} \frac{1}{\sqrt{t-s_{k}}}=\frac{(2 \sqrt{t})^{k}}{k !} .
$$

Calling $B_{n}(\epsilon)=\max _{k \leqslant n}(n \epsilon)^{k}$, 


$$
v_{n}(t) \leqslant B_{n}(\epsilon)\left[(1+2 t) \mathrm{e}^{\kappa t}\right]^{n} \sum_{k=0}^{n}\left(\begin{array}{l}
n \\
k
\end{array}\right)[2 c \sqrt{t}]^{k} C^{n-k} \leqslant B_{n}(\epsilon)\left[\mathrm{e}^{\kappa t}(1+2 t)(C+2 c \sqrt{t})\right]^{n} .
$$

Finally, $B_{n}(\epsilon) \leqslant 1$ for $n \leqslant \epsilon^{-1}$ while for $n>\epsilon^{-1}$ (and $\epsilon$ small) $B_{n}(\epsilon) \leqslant \epsilon^{n} n ! \mathrm{e}^{n}$ (indeed, by the Stirling formula, see (A.9), $n ! \mathrm{e}^{n} \geqslant n^{n}$ ). Hence (A.10), pending the validity of (A.13), which we will prove next.

Proof of (A.13). Let $\omega=\sum_{i=1}^{n} 1_{x_{i}}$. Recalling (A.7), the last term can then be written as

$$
\kappa \sum_{y \in \Omega_{\epsilon}}\left\{\int_{0}^{t} \sum_{y_{1}, \ldots, y_{n}}\left\{\prod_{i=1}^{n} \pi_{t-s}\left(x_{i}, y_{i}\right)\right\}\left\{\sum_{i=1}^{n} \mathbf{1}_{y_{i}=y}\right\}\left\{\left[\sum_{i=1}^{n} \mathbf{1}_{y_{i}=y}\right]-1\right\} E_{\mu^{\epsilon}}^{\epsilon}\left(D_{\sum 1_{y_{i}}-1_{y}}\left(\eta_{s}^{+}\right)\right)\right\} .
$$

Since $\left\{\sum_{i=1}^{n} \mathbf{1}_{y_{i}=y}\right\}\left\{\left[\sum_{i=1}^{n} \mathbf{1}_{y_{i}=y}\right]-1\right\}=\sum_{i, j \neq i} \mathbf{1}_{y_{i}=y_{j}=y}$, denoting by $\sum_{y_{1}, \ldots, y_{j}, \ldots, y_{n}}$ the sum with $y_{j}$ missing, i.e. only over $y_{1}, \ldots, y_{j-1}, y_{j+1}, \ldots, y_{n}$, we get

$$
\begin{aligned}
& \kappa \sum_{i, j \neq i} \sum_{y_{1}, \ldots, y_{n}} \mathbf{1}_{y_{i}=y_{j}} \int_{0}^{t}\left\{\prod_{\ell=1}^{n} \pi_{t-s}\left(x_{\ell}, y_{\ell}\right)\right\} E_{\mu^{\epsilon}}^{\epsilon}\left(D_{\sum_{\ell \neq j} 1_{y_{\ell}}}\left(\eta_{s}^{+}\right)\right) \\
& \leqslant \kappa \sum_{i, j \neq i} \sum_{y_{1}, \ldots, y_{j}, \ldots, y_{n}} \int_{0}^{t}\left\{\prod_{\ell \neq j} \pi_{t-s}\left(x_{\ell}, y_{\ell}\right)\right\}\left\{\sup _{x, y} \pi_{t-s}(x, y)\right\} E_{\mu^{\epsilon}}^{\epsilon}\left(D_{\sum_{\ell \neq j} 1_{y_{\ell}}}\left(\eta_{s}^{+}\right)\right)
\end{aligned}
$$

and since

$$
\kappa \sup _{x, y} \pi_{t-s}(x, y) \leqslant c \frac{\epsilon}{\sqrt{t-s}} \leqslant c \epsilon n(n-1) \int_{0}^{t} \frac{1}{\sqrt{t-s}} \sup _{\omega^{*}:\left|\omega^{*}\right|=n-1} \sum_{\omega^{\prime}:\left|\omega^{\prime}\right|=n-1} \pi_{t-s}^{(0)}\left(\omega^{*}, \omega^{\prime}\right) E_{\mu^{\epsilon}}^{\epsilon}\left(D_{\omega^{\prime}}\left(\eta_{s}^{+}\right)\right)
$$

which thus completes the proof of (A.13).

Proof of Theorem 8.1. We complete here the proof of Theorem 8.1 by proving (8.3) and (8.4). By (A.2),

$$
E_{\mu^{\epsilon}}^{\epsilon}\left(\mathrm{e}^{b \eta_{t}^{+}(x)}\right)=\sum_{n=0}^{\infty} \frac{\left(\mathrm{e}^{b}-1\right)^{n}}{n !} E_{\mu^{\epsilon}}^{\epsilon}\left(D_{\omega_{n, x}}\left(\eta_{t}^{+}\right)\right),
$$

where $\omega_{n, x}=n 1_{x}$ is the configuration with $n$ particles, all at $x$. By (A.10),

$$
\begin{aligned}
E_{\mu^{\epsilon}}^{\epsilon}\left(\mathrm{e}^{b \eta_{t}^{+}(x)}\right) & \leqslant \sum_{n=0}^{\infty} \frac{\left(\mathrm{e}^{b}-1\right)^{n}}{n !}\left[(1+2 t) \mathrm{e}^{\kappa t}(C+2 c \sqrt{t})\right]^{n}+\sum_{n \geqslant \epsilon^{-1}}\left(\mathrm{e}\left(\mathrm{e}^{b}-1\right)\left[(1+2 t) \mathrm{e}^{\kappa t}(C+2 c \sqrt{t}) \epsilon\right]\right)^{n} \\
& \leqslant 2 \exp \left\{\left(\mathrm{e}^{b}-1\right) \mathrm{e}^{\kappa t}(1+2 t)(C+2 c \sqrt{t})\right\}
\end{aligned}
$$

for $\epsilon$ small enough, which proves (8.1).

We will next prove that for any $\tau>0$ there is $c$ so that, for any $t \leqslant \tau$,

$$
E_{\mu^{\epsilon}}^{\epsilon}\left(\left|u^{\epsilon}\left(x, \eta_{t}^{+}\right)-E_{\mu^{\epsilon}}^{\epsilon}\left(u^{\epsilon}\left(x, \eta_{t}^{+}\right)\right)\right|^{2}\right) \leqslant c \epsilon^{(1-\alpha) d} .
$$

Proceeding as in the proof of Theorem 5.1,

$$
\begin{aligned}
& \text { 1.h.s. of (A.14) }=\sum_{z, z^{\prime}} p_{\alpha}(x, z) p_{\alpha}\left(x, z^{\prime}\right) V_{2}\left(z, z^{\prime}, t\right)+\sum_{z} p_{\alpha}(x, z)^{2} E_{\mu^{\epsilon}}^{\epsilon}\left(\left(\eta_{t}^{+}(z)\right)\right), \\
& V_{2}\left(z, z^{\prime}, t\right)=E_{\mu^{\epsilon}}^{\epsilon}\left(D_{\omega_{z, z^{\prime}}}\left(\eta_{t}^{+}\right)-E_{\mu^{\epsilon}}^{\epsilon}\left(D_{\omega_{z}}\left(\eta_{t}^{+}\right)\right) E_{\mu^{\epsilon}}^{\epsilon}\left(D_{\omega_{z^{\prime}}}\left(\eta_{t}^{+}\right)\right)\right) .
\end{aligned}
$$

Since $L^{(\eta,+)} d_{1}(\eta(x))=\kappa d_{1}(\eta(x))$ and $L^{(\eta,+)} d_{2}(\eta(x))=2 \kappa\left[d_{2}(\eta(x))+\eta(x)\right]$, 


$$
\begin{aligned}
V_{2}\left(z, z^{\prime}, t\right) \leqslant & \sum_{z_{1}, z_{1}^{\prime}} \pi_{\epsilon^{-2} t}\left(z, z_{1}\right) \pi_{\epsilon^{-2} t}\left(z^{\prime}, z_{1}^{\prime}\right) V_{2}\left(z_{1}, z_{1}^{\prime}, 0\right) \\
& +2 \kappa \int_{0}^{t}\left\{\sum_{z_{1}, z_{1}^{\prime}} \pi_{\epsilon^{-2} t}\left(z, z_{1}\right) \pi_{\epsilon^{-2} t}\left(z^{\prime}, z_{1}^{\prime}\right) V_{2}\left(z_{1}, z_{1}^{\prime}, s\right)\right. \\
& \left.+\sum_{z_{1}} \pi_{\epsilon^{-2+2 \alpha}+\epsilon^{-2}(t-s)}\left(z, z_{1}\right) \pi_{\epsilon^{-2+2 \alpha}+\epsilon^{-2}(t-s)}\left(z^{\prime}, z_{1}\right) E_{\mu^{\epsilon}}^{\epsilon}\left(\eta_{s}^{+}\left(z_{1}\right)\right)\right\}
\end{aligned}
$$

which iterated yields

$$
\begin{aligned}
\text { 1.h.s. of (A.14) } \leqslant & \sum_{z} p_{\alpha}(x, z)^{2} E_{\mu^{\epsilon}}^{\epsilon}\left(\eta_{t}^{+}(z)\right)+\mathrm{e}^{2 \kappa t} \sum_{z} \pi_{\epsilon^{-2+2 \alpha}+\epsilon^{-2}\left(t+t_{0}\right)}(x, z)^{2} \eta_{-t_{0}}(z) \\
& +2 \kappa \sum_{z} \int_{0}^{t} \mathrm{e}^{2 \kappa(t-s)} \pi_{\epsilon^{-2+2 \alpha}+\epsilon^{-2}(t-s)}(x, z)^{2} E_{\mu^{\epsilon}}^{\epsilon}\left(\eta_{s}^{+}(z)\right) .
\end{aligned}
$$

Using the inequality $E_{\mu^{\epsilon}}^{\epsilon}\left(\eta_{t}^{+}(z)\right) \leqslant C \mathrm{e}^{\kappa t}$, we then get (A.14).

Proof of (10.9). The expectation in (10.9) is computed by first conditioning on $\eta_{t}, \xi_{t}$, the conditional expectation is explicitly written in (10.8). According to (10.9) we will restrict to $\eta_{t}$ such that $u^{\epsilon}\left(x, \eta_{t}\right) \leqslant M, u^{\epsilon}\left(y, \eta_{t}\right) \leqslant M$ and we will prove that there is a constant $c$ depending on $m, n$ and $t$, so that

$$
\begin{aligned}
& \left|u^{\epsilon}\left(x, \eta_{t}\right)^{n} u^{\epsilon}\left(y, \eta_{t}\right)^{m}-\sum_{\omega_{n}^{\prime}, \omega_{m}^{\prime}} \pi_{\epsilon^{-2+2 \alpha}}^{(0)}\left(\omega_{n, x}, \omega_{n}^{\prime}\right) \pi_{\epsilon^{-2+2 \alpha}}^{(0)}\left(\omega_{m, y}, \omega_{m}^{\prime}\right) D_{\omega_{n}^{\prime}+\omega_{m}^{\prime}}\left(\eta_{t}\right)\right| \leqslant c \epsilon^{(1-\alpha) d}, \\
& \mid u^{\epsilon}\left(y, \eta_{t}\right)^{m} \sum_{\omega_{n}^{\prime}} \pi_{\epsilon^{-2+2 \alpha}}^{(0)}\left(\omega_{n, x}, \omega_{n}^{\prime}\right) D_{\omega_{n}^{\prime}}\left(\eta_{t}\right) \\
& \quad-\sum_{\omega_{n}^{\prime}, \omega_{m}^{\prime}} \pi_{\epsilon^{-2+2 \alpha}}^{(0)}\left(\omega_{n, x}, \omega_{n}^{\prime}\right) \pi_{\epsilon^{-2+2 \alpha}}^{(0)}\left(\omega_{m, y}, \omega_{m}^{\prime}\right) D_{\omega_{n}^{\prime}+\omega_{m}^{\prime}}\left(\eta_{t}\right) \mid \leqslant c \epsilon^{(1-\alpha) d}, \\
& \mid \sum_{\omega_{m}^{\prime}} \pi_{\epsilon^{-2+2 \alpha}}^{(0)}\left(\omega_{m, y}, \omega_{m}^{\prime}\right) D_{\omega_{m}^{\prime}}\left(\eta_{t}\right) u^{\epsilon}\left(x, \eta_{t}\right)^{n} \\
& \quad-\sum_{\omega_{n}^{\prime}, \omega_{m}^{\prime}} \pi_{\epsilon^{-2+2 \alpha}}^{(0)}\left(\omega_{n, x}, \omega_{n}^{\prime}\right) \pi_{\epsilon^{-2+2 \alpha}}^{(0)}\left(\omega_{m, y}, \omega_{m}^{\prime}\right) D_{\omega_{n}^{\prime}+\omega_{m}^{\prime}}\left(\eta_{t}\right) \mid \leqslant c \epsilon^{(1-\alpha) d} .
\end{aligned}
$$

(10.9) then follows from (10.8) and (A.15)-(A.17) and hence it will suffice to prove the latter. As their proofs are structurally the same we will only prove (A.17).

We fix $\omega_{m}^{\prime}$ in (A.17) and expand the term $u^{\epsilon}\left(x, \eta_{t}\right)^{n}=\left\{\sum_{z} p_{\alpha}(x, z) \eta_{t}(z)\right\}^{n}$. We distinguish the cases of single occupancy from the others which we will prove to be negligible. We thus write

$$
u^{\epsilon}\left(x, \eta_{t}\right)^{n}=\sum_{z_{1} \cdots z_{n} \in X_{n, \omega_{m}^{\prime}, \neq}} \prod_{i=1}^{n} p_{\alpha}\left(x, z_{i}\right) \eta_{t}\left(z_{i}\right)+\sum_{z_{1} \cdots z_{n} \notin X_{n, \omega_{m}^{\prime}, \neq}} \prod_{i=1}^{n} p_{\alpha}\left(x, z_{i}\right) \eta_{t}\left(z_{i}\right),
$$

where $X_{n, \omega_{m}^{\prime}, \neq}=\left\{z_{1} \cdots z_{n}: \omega_{m}^{\prime}\left(z_{i}\right)=0, i=1, \ldots, n ; z_{i} \neq z_{j}\right.$ for all $\left.i \neq j\right\}$. Thus

$$
\begin{aligned}
& u^{\epsilon}\left(x, \eta_{t}\right)^{n}=T_{\omega_{m}^{\prime}, x}+S_{\omega_{m}^{\prime}, x}, \quad T_{\omega_{m}^{\prime}, x}=\sum_{z_{1} \cdots z_{n} \in X_{n, \omega_{m}^{\prime}, \neq}}\left\{\prod_{i=1}^{n} p_{\alpha}\left(x, z_{i}\right)\right\} D_{\sum_{i} 1_{z_{i}}}\left(\eta_{t}\right), \\
& S_{\omega_{m}^{\prime}, x}=\sum_{z_{1} \cdots z_{n} \notin X_{n, \omega_{m}^{\prime}, \neq}} \prod_{i=1}^{n} p_{\alpha}\left(x, z_{i}\right) \eta_{t}\left(z_{i}\right) .
\end{aligned}
$$


We will next prove that the term with $S_{\omega_{m}^{\prime}, x}$ is negligible.

$$
\begin{aligned}
S_{\omega_{m}^{\prime}, x} \leqslant & \sum_{i=1}^{n}\left\{\sum_{z_{i}: \omega_{m}^{\prime}\left(z_{i}\right) \geqslant 1} p_{\alpha}\left(x, z_{i}\right) \eta_{t}\left(z_{i}\right)\right\} \sum_{z_{1} \cdots z_{i} \cdots z_{n}} \prod_{i \neq j} p_{\alpha}\left(x, z_{j}\right) \eta_{t}\left(z_{j}\right) \\
& +\sum_{i \neq j}\left\{\sum_{z: \omega_{m}^{\prime}(z)=0} p_{\alpha}(x, z)^{2} \eta_{t}(z)^{2}\right\} \sum_{z_{1} \cdots z_{i} \cdots z_{j} \cdots z_{n}} \prod_{\ell \neq i, j} p_{\alpha}\left(x, z_{\ell}\right) \eta_{t}\left(z_{\ell}\right) .
\end{aligned}
$$

Recalling that $u^{\epsilon}\left(x, \eta_{t}\right) \leqslant M$, see the beginning of this proof,

$$
S_{\omega_{m}^{\prime}, x} \leqslant M^{n-1} \sum_{i=1}^{n} \sum_{z_{i}: \omega_{m}^{\prime}\left(z_{i}\right) \geqslant 1} p_{\alpha}\left(x, z_{i}\right) \eta_{t}\left(z_{i}\right)+M^{n-2} \sum_{i \neq j}\left\{\sum_{z: \omega_{m}^{\prime}(z)=0} p_{\alpha}(x, z)^{2} \eta_{t}(z)^{2}\right\} .
$$

When multiplied by $D_{\omega_{m}^{\prime}}\left(\eta_{t}\right)$ we will have terms $p d_{k}(p)$ and $p^{2} d_{k}(p), p=\eta_{t}\left(z_{i}\right)$ and $k \omega_{m}^{\prime}\left(z_{i}\right) \geqslant 1$. We have

$$
p d_{k}(p)=d_{k+1}(p)+k d_{k}(p), \quad p^{2} d_{k}(p)=d_{k+2}(p)+(2 k+1) d_{k+1}(p)+k^{2} d_{k}(p) .
$$

Since $E_{\mu^{\epsilon}}^{\epsilon}\left(D_{\omega}\left(\eta_{t}\right)\right) \leqslant c(t)^{|\omega|}$,

$$
\begin{aligned}
E_{\mu^{\epsilon}}^{\epsilon}\left(\mathbf{1}_{u^{\epsilon}\left(x, \eta_{t}\right) \leqslant M} D_{\omega_{m}^{\prime}}\left(\eta_{t}\right) S_{\omega_{m}^{\prime}, x}\right) \leqslant & M^{n-1} n\left(m c \epsilon^{(1-\alpha) d}\right)\left[c(t)^{m+1}+c(t)^{m} m\right] \\
& +M^{n-2} n(n-1) c \epsilon^{(1-\alpha) d}\left[c(t)^{2}+(2 m+1) c(t)+m^{2}\right] c(t)^{m}
\end{aligned}
$$

so that this term is bounded by $c^{\prime} \epsilon^{(1-\alpha) d}$. The term with $T_{\omega_{m}^{\prime}, x}$, cf. (A.18), is

$$
\sum_{\omega_{m}^{\prime}, \omega_{n}^{\prime \prime}} \mathbf{1}_{\omega_{n}^{\prime \prime} \leqslant 1, \omega_{n}^{\prime \prime} \omega_{m}^{\prime} \equiv 0} \pi_{\epsilon^{-2+2 \alpha}}^{(0)}\left(\omega_{n, x}, \omega_{n}^{\prime \prime}\right) \pi_{\epsilon^{-2+2 \alpha}}^{(0)}\left(\omega_{m, y}, \omega_{m}^{\prime}\right) E_{\mu^{\epsilon}}^{\epsilon}\left(\mathbf{1}_{u^{\epsilon}\left(x, \eta_{t}\right) \leqslant M} \mathbf{1}_{u^{\epsilon}\left(y, \eta_{t}\right) \leqslant M} D_{\omega_{n}^{\prime \prime}+\omega_{m}^{\prime}}\left(\eta_{t}\right)\right),
$$

where $\omega_{n}^{\prime \prime} \leqslant 1, \omega_{n}^{\prime \prime} \omega_{m}^{\prime} \equiv 0$ is the set where for all $z, \omega_{n}^{\prime \prime}(z) \leqslant 1$ and $\omega_{n}^{\prime \prime}(z) \omega_{m}^{\prime}(z)=0$. Thus

$$
\text { 1.h.s. of }(\mathrm{A} .17) \leqslant\left|\sum_{\omega_{m}^{\prime},} \pi_{\epsilon^{-2+2 \alpha}}^{(0)}\left(\omega_{m, y}, \omega_{m}^{\prime}\right) \sum_{\omega_{n}^{\prime} \in X_{n, \omega_{m}^{\prime}, \neq}} \pi_{\epsilon^{-2+2 \alpha}}^{(0)}\left(\omega_{n, x}, \omega_{n}^{\prime}\right) D_{\omega_{n}^{\prime}+\omega_{m}^{\prime}}\left(\eta_{t}\right)\right|+c^{\prime} \epsilon^{(1-\alpha) d} \text {. }
$$

As before we fix $\omega_{m}^{\prime}$ and we bound the sum over $\omega_{n}^{\prime} \in X_{n, \omega_{m}^{\prime}} \neq \neq$ we distinguish the cases where a particle in $\omega_{n}^{\prime}$ is in a site $z_{i}: \omega^{\prime}\left(z_{i}\right) \geqslant 1$ and then the case of a coincidence $z_{i}=z_{j}, i \neq j, z_{i}, z_{j} \in \omega_{n}^{\prime}$. As before the probability of the former is in the end bounded by $c n m \epsilon^{(1-\alpha) d}$, while the latter is bounded by $c n(n-1) \epsilon^{(1-\alpha) d}$. In this way we derive (A.17). (A.15), (A.16) are bounded similarly and (10.9) is proved.

\section{Appendix B. Stirring process}

In $[6,7]$ it is proved that:

Theorem B.1. Let $p_{t}^{(\infty)}(\underline{x}, \underline{y})$ and $\pi_{t}^{(\infty)}(\underline{x}, \underline{y})$ denote the kernels of the semigroups of the stirring and of the independent processes in the whole $\bar{Z}^{d}$. Then, given any $n \geqslant 1$ (but the case $n=1$ is trivial, because $p_{t}(x, y) \equiv \pi_{t}(x, y)$ ), any dimension $d \geqslant 1$, there is $c$ so that, for any $\underline{x},|\underline{x}|=n$,

$$
\sum_{\underline{y}}\left|p_{t}^{(\infty)}(\underline{x}, \underline{y})-\pi_{t}^{(\infty)}(\underline{x}, \underline{y})\right| \leqslant c t^{-1 / 12} .
$$

The bound (B.1) cannot hold in our case where the processes are defined on the torus $\Omega_{\epsilon}$, because $p_{t}(\underline{x}, y)$ and $\pi_{t}(\underline{x}, \underline{y})$ have different limits as $t \rightarrow \infty$ : the former converges to the uniform distribution of $n$ distinct sites in $\Omega_{\epsilon}$ while in the latter the exclusion condition is dropped. However since

$$
\sum_{y:|y-x|>\epsilon^{-1}} p_{t}(x, y) \leqslant c \frac{\mathrm{e}^{-\epsilon^{-2} / t}}{\sqrt{t}} .
$$


(B.1) remains valid for the processes in $\Omega_{\epsilon}$ provided $t \leqslant \epsilon^{-2 a} \tau$ and with $c$ in (B.1) dependent on $\tau$ :

$$
\sup _{\epsilon>0} \sup _{t \leqslant \epsilon^{-2 a} \tau} \sup _{|\underline{x}|=n} t^{1 / 12} \sum_{\underline{y}}\left|p_{t}(\underline{x}, \underline{y})-\pi_{t}^{(0)}(\underline{x}, \underline{y})\right| \leqslant c_{n}(\tau) .
$$

Proof of Theorem 5.1. (Relative to the $\xi$ particles.) By definition

$$
\mu^{\epsilon}(\xi(x))=\sum_{y} \pi_{t_{0} \epsilon^{-2 a}}(x, y) \xi_{-t_{0}}(y)
$$

hence

$$
\epsilon^{-a}\left|\nabla \mu^{\epsilon}(\xi(x))\right| \leqslant \frac{c}{\sqrt{t_{0}}}
$$

uniformly in $\epsilon$ and $x \in \Omega_{\epsilon}$, which proves the second inequality in (5.5). We have

$$
\begin{aligned}
\left|v^{\epsilon}(x, \xi)-V_{0}^{\epsilon}(x)\right|^{2}= & \sum_{y} q_{\beta}(x, y)^{2}\left[\xi(y)-\mu^{\epsilon}(\xi(y))\right]^{2} \\
& +\sum_{y \neq z} q_{\beta}(x, y) q_{\beta}(x, z)\left[\xi(y)-\mu^{\epsilon}(\xi(y))\right]\left[\xi(z)-\mu^{\epsilon}(\xi(z))\right] .
\end{aligned}
$$

The $\mu^{\epsilon}$ expectation of the first term is bounded by $c \epsilon^{a-\beta}$. The expectation of the second term is computed using (B.2) and it is then bounded by $c\left[t_{0} \epsilon^{-2 a}\right]^{-1 / 12}$. The second inequality in (5.4) is proved.

Proof of (11.21). By (9.1),

$$
\epsilon^{-2 a}\left|A_{t_{N}, t_{N-1}}\left(\eta_{t_{N-1}}\right)-A_{t_{N}, 0}\left(\eta_{0}\right)\right| \leqslant c \epsilon^{2 \gamma+2 \beta-2 a}
$$

which is the first factor in (11.21). The proof of (11.21) then follows from the following theorem:

Theorem B.2. For any $n>1$ there is $c$ so that for any $\underline{x},|\underline{x}|=n, t \leqslant \epsilon^{-2} \tau$,

$$
\sup _{\|\psi\|_{\infty} \leqslant 1}\left|\sum_{\underline{z}} p_{t}(\underline{x}, \underline{z}) L^{\mathrm{st}} \psi(\underline{z})\right| \leqslant \frac{c}{t^{1 / 2+1 / 12}},
$$

where the sup is over all functions $\psi(\underline{z}),|\underline{z}|=n$, which are bounded by 1 .

Proof. The following bound can be found in the literature [5]: there is $c$ so that, for any $z, z^{\prime},\left|z-z^{\prime}\right|=1$, and any $t \leqslant \epsilon^{-2 a} \tau$,

$$
\sum_{\underline{y}}\left|p_{t}\left(\underline{x}^{\left(z, z^{\prime}\right)}, \underline{y}\right)-p_{t}(\underline{x}, \underline{y})\right| \leqslant \frac{c}{\sqrt{t}},
$$

where $\underline{x}^{\left(z, z^{\prime}\right)}$ is defined before (11.5). Writing $\mathrm{e}^{L^{\mathrm{st}} t} L^{\mathrm{st}}=\mathrm{e}^{L^{\mathrm{st}} t / 2} L^{\mathrm{st}} \mathrm{e}^{L^{\mathrm{st}} t / 2}$ we get

$$
\sum_{\underline{y}} p_{t}(\underline{x}, \underline{y}) L^{\mathrm{st}} \psi(\underline{y})=\sum_{\underline{z}} p_{t / 2}(\underline{x}, \underline{z}) L^{\mathrm{st}} \phi(\underline{z}), \quad \phi(\underline{z}):=\sum_{\underline{y}} p_{t / 2}(\underline{z}, \underline{y}) \psi(\underline{y}) .
$$

Let $\nabla_{i}$ denote the gradient acting on the variable $z_{i}$ and $E_{+}$the set of all positively oriented unit vectors of $\mathbb{Z}^{d}$. Then

$$
\begin{aligned}
& L^{\mathrm{st}} \phi(\underline{z})=\sum_{i=1}^{n} \sum_{|e|=1} \mathbf{1}_{z_{j} \neq z_{i}+e, j \neq i}\left\{\phi\left(\underline{z}^{\left(z_{i}, z_{i}+e\right)}\right)-\phi(\underline{z})\right\}=T_{1}+T_{2}, \\
& T_{1}(\underline{z})=-\sum_{i}^{n} \sum_{e \in \mathcal{E}_{+}} \mathbf{1}_{z_{j} \neq z_{i} \pm e, j \neq i}\left[(-e) \cdot \nabla_{i}\right]\left[e \cdot \nabla_{i}\right] \phi(\underline{z}), \\
& T_{2}(\underline{z})=\sum_{i, j} \sum_{|e|=1} \mathbf{1}_{z_{j}=z_{i}-e, \mathbf{1}_{z_{k} \neq z_{i}+e, k \neq i}\left\{\phi\left(\underline{z}^{\left(z_{i}, z_{i}+e\right)}\right)-\phi(\underline{z})\right\}}
\end{aligned}
$$


From (B.4), after "integrating by parts",

$$
\sum_{\underline{z}} p_{t / 2}(\underline{x}, \underline{z}) T_{1}(\underline{z})=-\sum_{i}^{n} \sum_{e \in E_{+}} \sum_{\underline{z}}\left\{\left(e \cdot \nabla_{i}\right)\left[p_{t / 2}(\underline{x}, \underline{z}) \mathbf{1}_{z_{j} \neq z_{i} \pm e, j \neq i}\right]\right\}\left\{\left(e \cdot \nabla_{i}\right) \phi(\underline{z})\right\} .
$$

Recalling the definition of $\phi$ in (B.4) and using (B.3), we bound the gradient of $\phi$ by $c / \sqrt{t}$. The first factor in (B.6) is instead estimated using (B.2) to reduce to independent particles, and gives the desired bound $c t^{-1 / 2-1 / 12}$. The term with $T_{2}(\underline{z})$, see (B.5), is treated similarly and Theorem B.2 is proved.

\section{Appendix C. Proof of Theorem 6.1. First part}

In this and in the next three Appendices we will bound

$$
E_{\mu^{\epsilon}}^{\epsilon}\left(\sup _{\tau^{\prime} \leqslant \tau}\left|\int_{0}^{\tau}(L+D)\left\{\left\|u^{\epsilon}-U^{\epsilon}\right\|^{2}+\left\|v^{\epsilon}-V^{\epsilon}\right\|^{2}\right\}\right|\right) .
$$

We will start by an explicit computation of $(L+D)\left\|u^{\epsilon}-U^{\epsilon}\right\|^{2}$ and $(L+D)\left\|v^{\epsilon}-V^{\epsilon}\right\|^{2}$ obtaining expressions which are sums of Laplacian and Reaction terms. The latter are those produced by the $L^{(\eta, 0)}+D^{(\eta, 0)}$ and $L^{(\xi, 0)}+D^{(\xi, 0)}$, with $D^{(\eta, 0)}$ and $D^{(\xi, 0)}$ the Laplacian parts of $D, D$ is defined in Section 3. The Reaction terms instead are produced by $L^{(\eta,+)}+D^{(\eta,+)}$ and $L^{(\xi,+)}+D^{(\xi,+)}$ for the births and by $L^{(\eta,-)}+D^{(\eta,-)}$ and $L^{(\xi,-)}+D^{(\xi,-)}$ for the deaths, having denoted by $D^{(\eta, \pm)}$ and $D^{(\xi, \pm)}$ the reaction terms in $D$.

A finer classification of all the terms distinguishes those denoted by $\mathcal{B}_{i}^{\epsilon}$ which are bounded proportionally to $\left\|u^{\epsilon}-U^{\epsilon}\right\|^{2}+\left\|v^{\epsilon}-V^{\epsilon}\right\|^{2}$ and those which when inserted in (C.1) vanish as $\epsilon \rightarrow 0$. They will be generically called remainders. Among them we call $R_{i}^{\epsilon}$ and $\mathcal{C}_{i}^{\epsilon}$ those which can be estimated using the a priori bounds of Section $8, \mathcal{S}_{i}^{\epsilon}$ those bounded in Appendix D exploiting the results of Section 10. There are two more terms, $\mathcal{H}$ which is bounded in Remarks C.1 below using Corollary 8.2 and $\mathcal{Q}$, studied in Appendix E using the analysis in Section 11. In Appendix F we bound the martingale terms in (4.4), (4.5), thus completing the proof of Theorem 6.1.

In the sequel we will often drop $\epsilon$ from the notation.

\section{C.1. Laplacian terms}

A computation essentially similar to the one in the proof of Theorem 8.2 yields

$$
\epsilon^{-2}\left(L^{(\eta, 0)}+D^{(\eta, 0)}\right)\|u-U\|^{2}=-2\|u-U\|_{H_{1}^{2}}^{2}+R_{1}^{\epsilon},
$$

where $R_{1}^{\epsilon}$, defined in (8.11), vanishes as $\epsilon \rightarrow 0$, see (4.6), and for this reason is called a $R^{\epsilon}$ term. We will not take advantage of the negative sign of the $H_{1}^{2}$ term $-2\|u-U\|_{H_{1}^{2}}^{2}$, which will be just bounded by 0 .

In an analogous way,

$$
\epsilon^{-2 a}\left(L^{(\xi, 0)}+D^{(\xi, 0)}\right)\|v-V\|^{2}=-2 \epsilon^{-2 a}\left\langle\nabla(v-V), \mathrm{e}^{-U} \nabla(v-V)\right\rangle+2 \mathcal{H}+R_{3}^{\epsilon}+R_{4}^{\epsilon}+2 \mathcal{S}_{1}^{\epsilon}+2 \mathcal{C}_{1}^{\epsilon},
$$

where $R_{4}^{\epsilon}$ is defined in (8.20) and bounded in (8.21),

$$
\begin{aligned}
\mathcal{H}(\xi, \eta) & =\epsilon^{-2 a}\left\langle v-V,\left[\mathrm{e}^{-u}-\mathrm{e}^{-U}\right] \Delta v\right\rangle, \\
R_{3}^{\epsilon}(\xi, \eta) & =-2 \epsilon^{-2 a}\left\langle v-V,\left(\nabla \mathrm{e}^{-U}\right) \nabla(v-V)\right\rangle \\
\mathcal{S}_{1}^{\epsilon}(\xi, \eta) & =\sum_{e:|e|=1} \epsilon^{d} \sum_{x}[v(x)-V(x)] \epsilon^{-2 a} \sum_{z} e \cdot \nabla q_{\beta}(x, z)\left\{\xi(z)(1-\xi(z+e))\left[\mathbf{1}_{\eta(z+e)=0}-\mathrm{e}^{-u(z+e)}\right]\right\}, \\
\mathcal{C}_{1}^{\epsilon}(\xi, \eta) & =\epsilon^{-2 a} \sum_{e:|e|=1} \epsilon^{d} \sum_{x}[v(x)-V(x)] \sum_{z} \xi(z)(1-\xi(z+e))\left[\mathrm{e}^{-u(z+e)}-\mathrm{e}^{-u(x)}\right] e \cdot \nabla q_{\beta}(x, z) .
\end{aligned}
$$


Remarks C.1. From (8.27) we get that

$$
-2 \epsilon^{-2 a}\left\langle\nabla(v-V), \mathrm{e}^{-U} \nabla(v-V)\right\rangle+2 \mathcal{H} \leqslant c\left(\|u-U\|^{2}+\|v-V\|^{2}+\epsilon^{2-2 a}\|u\|_{H_{1}^{2}}\right) .
$$

From (8.15), (8.9) and (4.9) it follows that

$$
E_{\mu^{\epsilon}}^{\epsilon}\left(\sup _{\tau^{\prime} \leqslant \tau}\left|\int_{0}^{\tau^{\prime}} \mathcal{C}_{1}^{\epsilon}\left(\xi_{t}, \eta_{t}\right)\right|\right) \leqslant \epsilon^{-2 a} \sum_{e, z}\left|e \cdot \nabla q_{\beta}(x, z)\right| \int_{0}^{\tau} \epsilon^{d} \sum_{x}\left|\mathrm{e}^{-u(z+e)}-\mathrm{e}^{-u(x)}\right| \leqslant c \epsilon^{1-2 a} .
$$

In Appendix D we prove that

$$
\lim _{\epsilon \rightarrow 0} E_{\mu^{\epsilon}}^{\epsilon}\left(\sup _{\tau^{\prime} \leqslant \tau}\left|\int_{0}^{\tau^{\prime}} \mathcal{S}_{1}^{\epsilon}\left(\xi_{t}, \eta_{t}\right)\right|\right)=0 .
$$

Finally, since $\left|\nabla e^{-U}\right| \leqslant c \epsilon,\left|R_{3}^{\epsilon}\right| \leqslant c \epsilon^{1-2 a}$.

\section{C.2. The reactions terms}

- Birth process, $\eta$-particles.

$$
\left(L^{(\eta,+)}+D^{(\eta,+)}\right)\|u-U\|^{2}=2 \kappa\|u-U\|^{2}+R_{2}^{\epsilon},
$$

where $R_{2}^{\epsilon}(\eta)$ is defined in (8.12) and bounded by $c \epsilon^{(1-\alpha) d}$, see the proof of Theorem 8.2.

- Death process, $\eta$-particles.

$$
\left(L^{(\eta,-)}+D^{(\eta,-)}\right)\|u-U\|^{2}=\mathcal{T}+2\left\langle u-U,\left[F_{1}^{-}(U)+F_{2}^{-}(U) V\right]\right\rangle+R_{5}^{\epsilon}-2 \mathcal{S}_{2}^{\epsilon},
$$

where $R_{5}^{\epsilon}$ is defined in (8.13), its expectation being bounded by $c \epsilon^{(1-\alpha) d}$, see again the proof of Theorem 8.2. Shorthanding by $\chi_{M, z}$ the characteristic function that $u(z, \eta) \leqslant M$,

$$
\begin{aligned}
\mathcal{T}= & -2 \epsilon^{d} \sum_{x}[u(x)-U(x)] \sum_{z} p_{\alpha}(x, z) \chi_{M, z} F_{1}^{-}(u(z)) \\
& -2 \epsilon^{d} \sum_{x}[u(x)-U(x)] \sum_{z} p_{\alpha}(x, z) \xi(z) \chi_{M, z} F_{2}^{-}(u(z))
\end{aligned}
$$

and

$$
\begin{aligned}
\mathcal{S}_{2}^{\epsilon}(\xi, \eta)= & \epsilon^{d} \sum_{x}[u(x)-U(x)] \sum_{z} p_{\alpha}(x, z)\left\{\left[\eta(z) \kappa_{\eta(z)}-\chi_{M, z} F_{1}^{-}(u(z))\right]\right. \\
& \left.+\xi(z)\left[\eta(z)\left[\kappa_{\eta(z)+1}-\kappa_{\eta(z)}\right]-\chi_{M, z} F_{2}^{-}(u(z))\right]\right\} .
\end{aligned}
$$

We next write

$$
\begin{aligned}
\mathcal{T}= & -2\left\langle u-U, F_{1}^{-}(U)\right\rangle-2\left\langle u-U, F_{2}^{-}(U) V\right\rangle \\
& -2 \epsilon^{d} \sum_{x}[u(x)-U(x)] F_{2}^{-}(U(x))\left\{\sum_{z} p_{\alpha}(x, z) \xi(z)-V(x)\right\}-2 \mathcal{B}_{1}^{\epsilon}+R_{6}^{\epsilon},
\end{aligned}
$$

where

$$
\begin{aligned}
R_{6}^{\epsilon}= & -2 \epsilon^{d} \sum_{x}[u(x)-U(x)] \sum_{z} p_{\alpha}(x, z)\left\{\left[F_{1}^{-}(U(z))-F_{1}^{-}(U(x))\right]+\xi(z)\left[F_{2}^{-}(U(z))-F_{2}^{-}(U(x))\right]\right. \\
& \left.-\left(1-\chi_{M, z}\right)\left[F_{1}^{-}(U(z))+\xi(z) F_{2}^{-}(U(z))\right]\right\}
\end{aligned}
$$

and

$$
\begin{aligned}
\mathcal{B}_{1}^{\epsilon}= & \epsilon^{d} \sum_{x}[u(x)-U(x)] \sum_{z} p_{\alpha}(x, z) \chi_{M, z}\left\{\left[F_{1}^{-}(u(z))-F_{1}^{-}(U(z))\right]\right. \\
& \left.+\xi(z)\left[F_{2}^{-}(u(z))-F_{2}^{-}(U(z))\right]\right\} .
\end{aligned}
$$


The first two terms on the right-hand side of (C.7) simplify with the second term on the right-hand side of (C.5). We then add and subtract $v(x)$ in the third term on the right-hand side of (C.7), so that

$$
\left(L^{(\eta,-)}+D^{(\eta,-)}\right)\|u-U\|^{2}=-2 \mathcal{S}_{2}^{\epsilon}-2\left(\mathcal{B}_{0}^{\epsilon}+\mathcal{B}_{1}^{\epsilon}\right)-2 \mathcal{C}_{2}^{\epsilon}+R_{5}^{\epsilon}+R_{6}^{\epsilon}+R_{7}^{\epsilon},
$$

where

$$
\begin{aligned}
& \mathcal{B}_{0}^{\epsilon}=\left\langle u-U, F_{2}^{-}(U)(v-V)\right\rangle, \\
& \mathcal{C}_{2}^{\epsilon}(\xi, \eta)=\left\langle\left(p_{\alpha}-1\right) *\left[(u-U) F_{2}^{-}(U)\right], v\right\rangle, \\
& R_{7}^{\epsilon}(\xi, \eta)=2 \epsilon^{d} \sum_{x}[u(x)-U(x)] F_{2}^{-}(U(x)) \sum_{z}\left\{p_{\alpha}(x, z)-\sum_{y} p_{\alpha}(x, y) q_{\beta}(y, z)\right\} \xi(z) .
\end{aligned}
$$

Remarks C.2. In Appendix D we prove that

$$
\lim _{\epsilon \rightarrow 0} E_{\mu^{\epsilon}}^{\epsilon}\left(\sup _{\tau^{\prime} \leqslant \tau}\left|\int_{0}^{\tau^{\prime}} \mathcal{S}_{2}^{\epsilon}\left(\xi_{t}, \eta_{t}\right)\right|\right)=0
$$

Using Theorem 8.1 to control the large values of $u(x)$ and to bound the expectation of $1-\chi_{M, z}$,

$$
\sup _{t \leqslant \tau} E_{\mu^{\epsilon}}^{\epsilon}\left(\left|R_{6}^{\epsilon}\left(\eta_{t}, \xi_{t}\right)\right|\right) \leqslant c M \epsilon \sum_{z} p_{\alpha}(0, z)|z|+c \epsilon^{(1-\alpha) d} .
$$

The expression on the right-hand side of (C.8) is called $\mathcal{B}_{1}^{\epsilon}$ because $\left|\mathcal{B}_{1}^{\epsilon}\right| \leqslant c_{M}\left\|u^{\epsilon}-U^{\epsilon}\right\|^{2}, c_{M}$ a constant which depends on $M$. Similarly, $\left|\mathcal{B}_{0}^{\epsilon}\right| \leqslant c_{M}\left(\left\|u^{\epsilon}-U^{\epsilon}\right\|^{2}+\left\|v^{\epsilon}-V^{\epsilon}\right\|^{2}\right)$, since $F_{2}^{-}\left(U^{\epsilon}\right)$ is uniformly bounded in compact time intervals.

From (8.15) and (8.9) it follows that

$$
E_{\mu^{\epsilon}}^{\epsilon}\left(\sup _{\tau^{\prime} \leqslant \tau}\left|\int_{0}^{\tau^{\prime}} \mathcal{C}_{2}^{\epsilon}\left(\xi_{t}, \eta_{t}\right) \mathrm{d} t\right|\right) \leqslant c \epsilon .
$$

Calling $t=\epsilon^{-2+2 \alpha}, s=\epsilon^{-2 a+2 \beta}$, since

$$
\sum_{z}\left|p_{\alpha} * q_{\beta}(x, z)-p_{\alpha}(x, z)\right|=\sum_{z}\left|\pi_{t+s}(x, z)-\pi_{t}(x, z)\right| \leqslant c \frac{s}{t}=c \epsilon^{2(1-\alpha-a+\beta)}
$$

the right-hand side of (C.10) is indeed a $R$-term.

- Birth process, $\xi$ particles.

$$
\left(L^{(\xi,+)}+D^{(\xi,+)}\right)\|v-V\|^{2}=\mathcal{T}_{1}-2 \kappa^{\prime}\left\langle v-V, V(1-V) \mathrm{e}^{-U}\right\rangle+R_{8}^{\epsilon},
$$

where $R_{8}^{\epsilon}$ is defined in (8.23) and

$$
\mathcal{T}_{1}=2 \kappa^{\prime} \epsilon^{d} \sum_{x}[v(x)-V(x)] \frac{1}{2 d} \sum_{e:|e|=1} \sum_{z} q_{\beta}(x, z+e) \xi(z)[1-\xi(z+e)] \mathbf{1}_{\eta(z+e)=0 .}
$$

By adding and subtracting $\mathrm{e}^{-u(z+e)}$ and calling

$$
\mathcal{S}_{3}^{\epsilon}=\epsilon^{d} \sum_{x}[v(x)-V(x)] \frac{1}{2 d} \sum_{e:|e|=1} \sum_{z} q_{\beta}(x, z+e) \xi(z)[1-\xi(z+e)]\left[\mathbf{1}_{\eta(z+e)=0}-\mathrm{e}^{-u(z+e)}\right]
$$

we get

$$
\begin{aligned}
\mathcal{T}_{1}= & 2 \kappa^{\prime} \mathcal{S}_{3}^{\epsilon}+2 \epsilon^{d} \sum_{x}[v(x)-V(x)] \frac{\kappa^{\prime}}{2 d} \sum_{e:|e|=1} \sum_{z} q_{\beta}(x, z+e) \xi(z)[1-\xi(z+e)] \\
& \times\left\{\mathrm{e}^{-U(x)}+\left[\mathrm{e}^{-U(z+e)}-\mathrm{e}^{-U(x)}\right]+\left[\mathrm{e}^{-u(z+e)}-\mathrm{e}^{-U(z+e)}\right]\right\} \\
= & 2 \kappa^{\prime} \mathcal{S}_{3}^{\epsilon}+\mathcal{T}_{2}+R_{10}^{\epsilon}+\mathcal{B}_{2}^{\epsilon},
\end{aligned}
$$


where $\mathcal{T}_{2}, R_{10}^{\epsilon}$ and $\mathcal{B}_{2}^{\epsilon}$ identify the corresponding terms in the previous line ( $\mathcal{B}_{2}^{\epsilon}$ is explicitly written in (C.14) below). We next write

$$
\begin{aligned}
\mathcal{T}_{2}= & 2 \epsilon^{d} \sum_{x}[v(x)-V(x)] \mathrm{e}^{-U(x)} \frac{\kappa^{\prime}}{2 d} \sum_{e:|e|=1} \sum_{z} q_{\beta}(x, z+e)\{V(x)[1-V(x)]+[v(x)[1-v(x+e)] \\
& -V(x)[1-V(x)]]+[\xi(z)[1-\xi(z+e)]-v(x)[1-v(x+e)]]\} \\
= & \mathcal{T}_{3}+\overline{\mathcal{B}}_{5}^{\epsilon}+2 \kappa^{\prime} \mathcal{Q}^{\epsilon},
\end{aligned}
$$

where, as before, $\mathcal{T}_{3}, \overline{\mathcal{B}}_{5}^{\epsilon}$ and $\mathcal{Q}^{\epsilon}$ identify the corresponding terms in the previous line, in particular $\mathcal{Q}^{\epsilon}$ is explicitly written in (C.13) below. Observe that $\mathcal{T}_{3}$ cancels with the first term on the right-hand side of (C.11). We rewrite $\overline{\mathcal{B}}_{5}^{\epsilon}$ as

$$
\begin{aligned}
\overline{\mathcal{B}}_{5}^{\epsilon}= & 2 \epsilon^{d} \sum_{x}[v(x)-V(x)] \mathrm{e}^{-U(x)} \frac{\kappa^{\prime}}{2 d} \sum_{e:|e|=1}\{[1-v(x+e)][v(x)-V(x)] \\
& +V(x)[V(x+e)-v(x+e)]+V(x)[V(x)-V(x+e)]\} \\
= & \mathcal{B}_{5}^{\epsilon}+R_{13}^{\epsilon},
\end{aligned}
$$

where $\mathcal{B}_{5}^{\epsilon}$ and $R_{13}^{\epsilon}$ identify the corresponding terms in the previous line.

Thus

$$
\left(L^{(\xi,+)}+D^{(\xi,+)}\right)\|v-V\|^{2}=\mathcal{B}_{5}^{\epsilon}+2 \kappa^{\prime} \mathcal{S}_{3}^{\epsilon}+2 \kappa^{\prime} \mathcal{Q}^{\epsilon}+\mathcal{B}_{2}^{\epsilon}+R_{8}^{\epsilon}+R_{10}^{\epsilon}+R_{13}^{\epsilon},
$$

where

$$
\begin{aligned}
& \mathcal{Q}^{\epsilon}=\epsilon^{d} \sum_{x}[v(x)-V(x)] \mathrm{e}^{-U(x)} \frac{1}{2 d} \sum_{e:|e|=1} \sum_{z} q_{\beta}(x, z+e)\{\xi(z)[1-\xi(z+e)]-v(z)[1-v(z+e)]\}, \\
& \mathcal{B}_{2}^{\epsilon}=\epsilon^{d} \sum_{x}[v(x)-V(x)] \frac{\kappa^{\prime}}{2 d} \sum_{e:|e|=1} \sum_{z} q_{\beta}(x, z+e) \xi(z)[1-\xi(z+e)]\left[\mathrm{e}^{-u(z+e)}-\mathrm{e}^{-U(z+e)}\right] .
\end{aligned}
$$

Remarks C.3. In Appendix D we prove that

$$
\lim _{\epsilon \rightarrow 0} E_{\mu^{\epsilon}}^{\epsilon}\left(\sup _{\tau^{\prime} \leqslant \tau}\left|\int_{0}^{\tau^{\prime}} \mathcal{S}_{3}^{\epsilon}\left(\xi_{t}, \eta_{t}\right)\right| \mathrm{d} t\right)=0 .
$$

We further observe that $\left|\mathcal{B}_{2}^{\epsilon}\right|+\left|\mathcal{B}_{5}^{\epsilon}\right| \leqslant c\left(\left\|u^{\epsilon}-U^{\epsilon}\right\|^{2}+\left\|v^{\epsilon}-V^{\epsilon}\right\|^{2}\right)$. In Appendix E we prove that

$$
\lim _{\epsilon \rightarrow 0} E_{\mu^{\epsilon}}^{\epsilon}\left(\sup _{\tau^{\prime} \leqslant \tau}\left|\int_{0}^{\tau^{\prime}} \mathcal{Q}^{\epsilon}\left(\xi_{t}, \eta_{t}\right)\right| \mathrm{d} t\right)=0 .
$$

- Death process, $\xi$-particles.

$$
\begin{aligned}
\left(L^{(\xi,-)}+D^{(\xi,-)}\right)\|v-V\|^{2}= & -2 \epsilon^{d} \sum_{x}[v(x)-V(x)] \sum_{z} q_{\beta}(x, z) \xi(z) \kappa_{\eta(z)+1} \\
& +2\left\langle v-V, V G^{-}(U)\right\rangle+R_{9}^{\epsilon}
\end{aligned}
$$

where $R_{9}^{\epsilon}$ is defined in (8.24). As before,

$$
\begin{aligned}
2 \epsilon^{d} & \sum_{x}[v(x)-V(x)] \sum_{z} q_{\beta}(x, z) \xi(z) \kappa_{\eta}(z)+1 \\
= & 2 \mathcal{S}_{4}+\epsilon^{d} \sum_{x}[v(x)-V(x)] \sum_{z} q_{\beta}(x, z) \xi(z) \\
& \times\left\{G^{-}(U(x))+\left[G^{-}(U(z))-G^{-}(U(x))\right]+\left[\chi_{M, z} G^{-}(u(z))-G^{-}(U(z))\right]\right\} \\
= & 2 \mathcal{S}_{4}^{\epsilon}+2\left\langle v-V, v G^{-}(U)\right\rangle+R_{12}^{\epsilon}+2 \mathcal{B}_{3}^{\epsilon},
\end{aligned}
$$


where

$$
\begin{aligned}
& \mathcal{S}_{4}=\epsilon^{d} \sum_{x}[v(x)-V(x)] \sum_{z} q_{\beta}(x, z) \xi(z)\left\{\kappa_{\eta(z)+1}-\chi_{M, z}(\eta) G^{-}(u(z))\right\}, \\
& \mathcal{B}_{3}=\epsilon^{d} \sum_{x}[v(x)-V(x)] \sum_{z} q_{\beta}(x, z) \xi(z) \chi_{M, z}(\eta)\left[G^{-}(u(z))-G^{-}(U(z))\right], \\
& R_{12}^{\epsilon}=-2 \epsilon^{d} \sum_{x}[v(x)-V(x)] \sum_{z} q_{\beta}(x, z) \xi(z)\left\{\left[G^{-}(U(z))-G^{-}(U(x))\right]-\left(1-\chi_{M, z}(\eta)\right) G^{-}(U(z))\right\} .
\end{aligned}
$$

We thus have

$$
\left(L^{(\xi,-)}+D^{(\xi,-)}\right)\|v-V\|^{2}=-2\left\langle v-V,[v-V] G^{-}(U)\right\rangle-2 \mathcal{S}_{4}-2 \mathcal{B}_{3}+R_{9}^{\epsilon}+R_{12}^{\epsilon} .
$$

Remarks C.4. Since $G^{-}\left(U^{\epsilon}\right)$ is uniformly bounded in compact time intervals, there is a constant $c_{M}$ (which depends on $M$ ), so that $\left|\mathcal{B}_{3}^{\epsilon}\right| \leqslant c_{M}\left(\left\|u^{\epsilon}-U^{\epsilon}\right\|^{2}+\left\|v^{\epsilon}-V^{\epsilon}\right\|^{2}\right)$. In Appendix D it is proven that the term $\mathcal{S}_{4}^{\epsilon}$ gives a vanishing contribution as in (C.15). Finally $R_{12}^{\epsilon}$ is an $R$ term because of (8.2).

\section{C.3. Estimates of the remainders}

From (C.2), (C.4) and (C.9) we get that

$$
(L+D)\|u-U\|^{2}=-2\|u-U\|_{H_{1}^{2}}^{2}+2 \kappa\|u-U\|^{2}-2\left(\mathcal{B}_{0}^{\epsilon}+\mathcal{B}_{1}^{\epsilon}\right)+\mathfrak{R}_{1}^{\epsilon},
$$

where

$$
\mathfrak{R}_{1}^{\epsilon}(\xi, \eta)=-2 \mathcal{S}_{2}^{\epsilon}-2 \mathcal{C}_{2}^{\epsilon}+\sum_{k=1,2,5,6,7} R_{k}^{\epsilon} .
$$

We now observe that the first term in (C.18) is non positive and that from Remarks C.2 we get that given any $\tau>0$ there are $c_{1}$ and $c_{2}$ so that

$$
\begin{aligned}
\left|\int_{0}^{\tau}(L+D)\left\{\left\|u^{\epsilon}\left(\eta_{t}\right)-U^{\epsilon}(t)\right\|^{2}\right\} \mathrm{d} t\right| \leqslant & \int_{0}^{\tau}\left\{c_{1}\left\|u^{\epsilon}\left(\eta_{t}\right)-U^{\epsilon}(t)\right\|^{2}+c_{2}\left\|v^{\epsilon}\left(\xi_{t}\right)-V^{\epsilon}(t)\right\|^{2}\right\} \\
& +\int_{0}^{\tau} \mathfrak{R}_{1}^{\epsilon}\left(\xi_{t}, \eta_{t}\right) \mathrm{d} t, \\
\lim _{\epsilon \rightarrow 0} E_{\mu^{\epsilon}}^{\epsilon}\left(\left|\sup _{\tau^{\prime} \leqslant \tau} \int_{0}^{\tau^{\prime}} \mathfrak{R}_{1}^{\epsilon}\left(\xi_{t}, \eta_{t}\right)\right|\right)=0 . &
\end{aligned}
$$

From (C.3), (C.12) and (C.17) we get that

$$
\begin{aligned}
(L+D)\|v-V\|^{2}= & -2 \epsilon^{-2 a}\left\langle\nabla(v-V), \mathrm{e}^{-U} \nabla(v-V)\right\rangle+2 \mathcal{H} \\
& -2\left\langle v-V,[v-V] G^{-}(U)\right\rangle+\mathcal{B}_{2}^{\epsilon}-2 \mathcal{B}_{3}^{\epsilon}+\mathcal{B}_{5}^{\epsilon}+\mathfrak{R}_{2}^{\epsilon},
\end{aligned}
$$

where

$$
\mathfrak{R}_{2}^{\epsilon}(\xi, \eta)=2\left(\mathcal{S}_{1}^{\epsilon}+\kappa^{\prime} \mathcal{S}_{3}^{\epsilon}-\mathcal{S}_{4}^{\epsilon}\right)+2 \mathcal{C}_{1}^{\epsilon}+2 \kappa^{\prime} \mathcal{Q}^{\epsilon}+\sum_{k=3,4,8,9,10,12,13} R_{k}^{\epsilon}
$$

We now observe that the third term is non negative and by Remarks C.1, C.3 and C.4 we get given any $\tau>0$ there are $c_{3}$ and $c_{4}$ so that 


$$
\begin{aligned}
\left|\int_{0}^{\tau}(L+D)\left\{\left\|v^{\epsilon}\left(\eta_{t}\right)-V^{\epsilon}(t)\right\|^{2}\right\} \mathrm{d} t\right| \leqslant & \int_{0}^{\tau}\left\{c_{3}\left\|u^{\epsilon}\left(\eta_{t}\right)-U^{\epsilon}(t)\right\|^{2}+c_{4}\left\|v^{\epsilon}\left(\xi_{t}\right)-V^{\epsilon}(t)\right\|^{2}\right\} \\
& +\int_{0}^{\tau} \Re_{2}^{\epsilon}\left(\xi_{t}, \eta_{t}\right) \mathrm{d} t
\end{aligned}
$$

\section{Appendix D. The $\mathcal{S}$ terms}

Denote by $X_{i}^{\epsilon}, i=1, \ldots, 4$, the terms in (4.4), (4.5) which contain $\mathcal{S}_{i}^{\epsilon}$ (their explicit expressions will be recalled below). The most dangerous one is $X_{1}^{\epsilon}$ because it appears with a divergent multiplicative factor $\epsilon^{-2 a}$, see (D.1). We thus start from this one, although the following analysis covers, except for some coefficients which are different, the easier term $\mathcal{S}_{3}^{\epsilon}$, where the dangerous factor $\epsilon^{-2 a}$ is absent.

Calling

$$
a_{1}^{\epsilon}(t, y)=\sum_{x,|e|=1}\left[v^{\epsilon}\left(x, \xi_{t}\right)-V^{\epsilon}(x, t)\right] e \cdot \nabla q_{\beta}(x, y-e) \xi_{t}(y-e)\left(1-\xi_{t}(y)\right)
$$

the term of the remainder containing $\mathcal{S}_{1}^{\epsilon}$ is

$$
X_{1}^{\epsilon}:=\epsilon^{-2 a} E_{\mu^{\epsilon}}^{\epsilon}\left(\sup _{\tau^{\prime} \leqslant \tau}\left|\epsilon^{d} \sum_{y} \int_{0}^{\tau^{\prime}} a_{1}^{\epsilon}(t, y)\left[\mathbf{1}_{\eta_{t}(y)=0}-\mathrm{e}^{-u^{\epsilon}\left(y, \eta_{t}\right)}\right]\right|\right)
$$

We write

$$
\int_{0}^{\tau} a_{1}^{\epsilon}(t, y) \mathbf{1}_{\eta_{t}(y)=0}=\int_{0}^{\tau} a_{1}^{\epsilon}\left(t+\epsilon^{2 \alpha}, y\right) \mathbf{1}_{\eta_{t+\epsilon^{2 \alpha}}(y)=0}+\int_{0}^{\epsilon^{2 \alpha}} a_{1}^{\epsilon}(t, y) \mathbf{1}_{\eta_{t}(y)=0}-\int_{\tau}^{\tau+\epsilon^{2 \alpha}} a_{1}^{\epsilon}(t, y) \mathbf{1}_{\eta_{t}(y)=0 .} .
$$

Denoting by $\|a\|:=\sup _{\epsilon \in(0,1)} \sup _{y \in \Omega_{\epsilon}} \sup _{t \leqslant \tau+\epsilon^{2 \alpha}}\left|a_{1}^{\epsilon}(t, y)\right|<\infty$,

$$
\left|\int_{0}^{\tau} a_{1}^{\epsilon}(t, y) \mathbf{1}_{\eta_{t}(y)=0}-\int_{0}^{\tau} a_{1}^{\epsilon}(t, y) \mathbf{1}_{\eta_{t+\epsilon^{2 \alpha}}(y)=0}\right| \leqslant \int_{0}^{\tau}\left|a_{1}^{\epsilon}\left(t+\epsilon^{2 \alpha}, y\right)-a_{1}^{\epsilon}(t, y)\right|+2\|a\| \epsilon^{2 \alpha} .
$$

We have that

$$
E_{\mu^{\epsilon}}^{\epsilon}\left(a_{1}^{\epsilon}\left(t+\epsilon^{2 \alpha}, y\right)-a_{1}^{\epsilon}(t, y)\right)=\int_{t}^{t+\epsilon^{2 \alpha}} E_{\mu^{\epsilon}}^{\epsilon}\left(\left(L^{(\xi)}+D^{(\xi)}\right) a_{1}^{\epsilon}(s, y)\right)
$$

and by Theorem 8.1,

$$
\epsilon^{-2 a} \epsilon^{d} \sum_{y} E_{\mu^{\epsilon}}^{\epsilon}\left(\int_{0}^{\tau}\left|a_{1}^{\epsilon}\left(t+\epsilon^{2 \alpha}, y\right)-a_{1}^{\epsilon}(t, y)\right|\right) \leqslant c \epsilon^{2(\alpha-2 a)}
$$

Then, calling

$$
f_{t}(y)=\left[\mathbf{1}_{\eta_{t+\epsilon^{2 \alpha}}(y)=0}-\mathrm{e}^{-u^{\epsilon}\left(y, \eta_{t}\right)}\right]
$$

from (D.2) and (D.3) we get that 


$$
X_{1}^{\epsilon} \leqslant \epsilon^{-2 a} E_{\mu^{\epsilon}}^{\epsilon}\left(\sup _{\tau^{\prime} \leqslant \tau}\left|\int_{0}^{\tau^{\prime}} \epsilon^{d} \sum_{y} a_{1}^{\epsilon}(t, y) f_{t}(y)\right|\right)+c \epsilon^{2(\alpha-a)}+2\|a\| \epsilon^{2(\alpha-2 a)}
$$

and with a new constant $c$

$$
\begin{aligned}
X_{1}^{\epsilon} & \leqslant \epsilon^{-2 a} E_{\mu^{\epsilon}}^{\epsilon}\left(\int_{0}^{\tau}\left|\epsilon^{d} \sum_{y} a_{1}^{\epsilon}(t, y) f_{t}(y)\right|\right)+c \epsilon^{2(\alpha-2 a)} \\
& \leqslant \epsilon^{-2 a} \int_{0}^{\tau} E_{\mu^{\epsilon}}^{\epsilon}\left(\left|\epsilon^{d} \sum_{y} a_{1}^{\epsilon}(t, y) f_{t}(y)\right|^{2}\right)^{1 / 2}+c \epsilon^{2(\alpha-2 a)} \\
& \leqslant \epsilon^{-2 a}\|a\| \tau \sup _{t \leqslant \tau\left|y-y^{\prime}\right| \geqslant \epsilon^{-1+\alpha / 2}}\left|E_{\mu^{\epsilon}}^{\epsilon}\left(f_{t}(y) f_{t}\left(y^{\prime}\right)\right)\right|^{1 / 2}+c \epsilon^{2(\alpha-2 a)}+\|a\| \tau \epsilon^{\alpha d / 4-2 a} .
\end{aligned}
$$

Indeed $\left|f_{t}(y)\right| \leqslant 1$, so that $\epsilon^{2 d} \sum_{\left|y-y^{\prime}\right|<\epsilon^{-1+\alpha / 2}}\left|f_{t}(y)\right|\left|f_{t}\left(y^{\prime}\right)\right| \leqslant \epsilon^{\alpha d / 2}$ and since there is a square root, the final bound goes like $\epsilon^{\alpha d / 4}$. By Theorem 10.1, $\left|E_{\mu^{\epsilon}}^{\epsilon}\left(f_{t}(y) f_{t}\left(y^{\prime}\right)\right)\right| \leqslant c \epsilon^{2 \alpha}$ so that $X_{1}^{\epsilon} \rightarrow 0$, because $\alpha>2 a$ and $\alpha>8 a / d$. We have thus concluded the analysis of $\mathcal{S}_{1}^{\epsilon}$ (and of $\mathcal{S}_{3}^{\epsilon}$, as well).

Recalling (C.16),

$$
\begin{aligned}
& X_{4}^{\epsilon}:=E_{\mu^{\epsilon}}^{\epsilon}\left(\sup _{\tau^{\prime} \leqslant \tau}\left|\int_{0}^{\tau^{\prime}} \epsilon^{d} \sum_{y} a_{4}^{\epsilon}(t, y)\left\{\left[\kappa_{\eta_{t}(y)+1}-\chi_{M, y} G^{-}\left(u^{\epsilon}\left(y, \eta_{t}\right)\right)\right]\right\}\right|\right), \\
& a_{4}^{\epsilon}(t, y)=\sum_{x}\left[v^{\epsilon}\left(x, \xi_{t}\right)-V^{\epsilon}(x, t)\right] q_{\beta}(x, y) \xi(y) .
\end{aligned}
$$

Analogously to (D.3), we get

$$
\begin{aligned}
\left|X_{4}^{\epsilon}\right| & \leqslant E_{\mu^{\epsilon}}^{\epsilon}\left(\sup _{\tau^{\prime} \leqslant \tau}\left|\int_{0}^{\tau^{\prime}} \epsilon^{d} \sum_{y}\left[\kappa_{\eta_{t+\epsilon^{2 \alpha}}(y)+1}-\chi_{M, y} G^{-}\left(u^{\epsilon}\left(y, \eta_{t}\right)\right)\right]\right|\right)+c \epsilon^{2 \alpha} \\
& \leqslant E_{\mu^{\epsilon}}^{\epsilon}\left(\int_{0}^{\tau} \epsilon^{d} \sum_{y}\left|\kappa_{\eta_{t+\epsilon^{2 \alpha}}(y)+1}-\chi_{M, y} G^{-}\left(u^{\epsilon}\left(y, \eta_{t}\right)\right)\right|\right)+c \epsilon^{2 \alpha} \\
& \leqslant E_{\mu^{\epsilon}}^{\epsilon}\left(\int_{0}^{\tau} \epsilon^{d} \sum_{y} \chi_{M, y}\left|\kappa_{\eta_{t+\epsilon^{2 \alpha}}(y)+1}-G^{-}\left(u^{\epsilon}\left(y, \eta_{t}\right)\right)\right|\right)+c\left[\epsilon^{2 \alpha}+\epsilon^{(1-\alpha) d / 2}\right]
\end{aligned}
$$

having used Cauchy-Schwartz and Theorem 8.1 in the last inequality. Hence

$$
\begin{aligned}
& X_{4}^{\epsilon} \leqslant \tau \sup _{t \leqslant \tau\left|y-y^{\prime}\right| \geqslant \epsilon^{-1+\alpha / 2}}\left|E_{\mu^{\epsilon}}^{\epsilon}\left(g_{t}(y) g_{t}\left(y^{\prime}\right)\right)\right|+c \epsilon^{2 \alpha}+c^{\prime} \epsilon^{\alpha d / 4-2 a}, \\
& g_{t}(y)=\chi_{M, y}\left[\kappa_{\eta_{t+\epsilon^{2 \alpha}}(y)+1}-G^{-}\left(u^{\epsilon}\left(z, \eta_{t}\right)\right)\right] .
\end{aligned}
$$

Since

$$
G^{-}(u)=\mathcal{E}_{u}\left(\kappa_{\eta(0)+1}\right), \quad \mathcal{E}_{u}(\eta(0))=u
$$

$\left(\mathcal{E}_{u}\right.$ is the expectation w.r.t. the Poisson law on $\mathbb{N}$ which has density $u$ ), by Theorem 10.2 we conclude that $X_{4}^{\epsilon} \rightarrow 0$.

We finally consider the remainder containing $\mathcal{S}_{2}^{\epsilon}$ : it consists of the sum of two terms, see (C.6), whose structures are essentially similar. For simplicity we only consider the first one. We have

$$
X_{2}^{\epsilon}:=E_{\mu^{\epsilon}}^{\epsilon}\left(\sup _{\tau^{\prime} \leqslant \tau}\left|\int_{0}^{\tau^{\prime}} \epsilon^{d} \sum_{x}\left[u^{\epsilon}\left(x, \eta_{t}\right)-U^{\epsilon}(x, t)\right] \sum_{z} p_{\alpha}(z-x)\left[\eta_{t}(z) \kappa_{\eta_{t}}(z)-\chi_{M, z} F_{1}^{-}\left(u^{\epsilon}\left(z, \eta_{t}\right)\right)\right]\right|\right),
$$


where

$$
F_{1}^{-}(u)=\mathcal{E}_{u}\left(\eta(0) \kappa_{\eta(0)}\right)
$$

and, again,

$$
\begin{aligned}
& X_{2}^{\epsilon} \leqslant\left. M \tau \sup _{t \leqslant \tau\left|y-y^{\prime}\right| \geqslant \epsilon^{-1+\alpha / 2}} \sup _{\mu^{\epsilon}}\left(h_{t}(y) h_{t}\left(y^{\prime}\right)\right)\right|^{1 / 2}+c \epsilon^{2 \alpha(7 / 4)}+c^{\prime} \epsilon^{\alpha d / 4-2 a}+c^{\prime \prime} \epsilon^{(1-\alpha) d / 2}, \\
& h_{t}(z)=\eta_{t+\epsilon^{2 \alpha}(z) \kappa_{\eta_{t+\epsilon^{2 \alpha}}(z)}-F_{1}^{-}\left(u^{\epsilon}\left(z, \eta_{t}\right)\right),}
\end{aligned}
$$

where the error $c \epsilon^{2 \alpha(7 / 4)}$ comes from having used Theorem 9.1 to express $u_{t+\epsilon^{2 \alpha}}^{\epsilon}(\cdot)$ as a linear combination of $u_{t}^{\epsilon}(\cdot)$. We have then used Cauchy-Schwartz and Theorem 8.1 to bound the contribution of $u^{\epsilon}>M$.

\section{Appendix E. The term $\mathcal{Q}$}

In this appendix we will prove that for any $\tau>0$,

$$
\lim _{\epsilon \rightarrow 0} E_{\mu^{\epsilon}}^{\epsilon}\left(\sup _{\tau^{\prime} \leqslant \tau}\left|\int_{0}^{\tau^{\prime}} \mathcal{Q}\left(\xi_{t}\right)\right|\right)=0
$$

with $\mathcal{Q}(\xi)$ defined in (C.13). We obviously have

$$
E_{\mu^{\epsilon}}^{\epsilon}\left(\sup _{\tau^{\prime} \leqslant \tau}\left|\int_{0}^{\tau^{\prime}} \mathcal{Q}\left(\xi_{t}\right)\right|\right) \leqslant E_{\mu^{\epsilon}}^{\epsilon}\left(\sup _{\tau^{\prime} \leqslant \tau} \int_{0}^{\tau^{\prime}}\left|\mathcal{Q}\left(\xi_{t}\right)\right|\right)=E_{\mu^{\epsilon}}^{\epsilon}\left(\int_{0}^{\tau}\left|\mathcal{Q}\left(\xi_{t}\right)\right|\right)=\tau \nu_{\tau}^{\epsilon}\left(\left|\mathcal{Q}\left(\xi_{0}\right)\right|\right),
$$

where $v_{\tau}^{\epsilon}$ is the probability measure defined in (11.1). Since $\left|v^{\epsilon}\left(x, \xi_{t}\right)-V^{\epsilon}(x, t)\right| \leqslant 1$ and $\nu_{\tau}^{\epsilon}$ is translational invariant,

$$
v_{\tau}^{\epsilon}\left(\left|\mathcal{Q}\left(\xi_{0}\right)\right|\right) \leqslant \frac{1}{2 d} \sum_{e:|e|=1}\left\{v^{\epsilon}\left(0, \xi_{0}\right)\left[1-v^{\epsilon}\left(e, \xi_{0}\right)\right]-\xi_{0}(0)\left[1-\xi_{0}(e)\right]\right\}
$$

hence

$$
E_{\mu^{\epsilon}}^{\epsilon}\left(\sup _{\tau^{\prime} \leqslant \tau}\left|\int_{0}^{\tau^{\prime}} \mathcal{Q}\left(\xi_{t}\right)\right|\right) \leqslant \tau \nu_{\tau}^{\epsilon}\left(\left|f^{\epsilon}\right|\right)
$$

where

$$
\begin{aligned}
f^{\epsilon}(\xi) & :=\left\{\sum_{z} \frac{1}{2 d} \sum_{e:|e|=1} q_{\beta}(0, z) \xi(z)[1-\xi(z+e)]\right\}-v^{\epsilon}(0, \xi)\left[1-v^{\epsilon}(e, \xi)\right] \\
& =\sum_{z} \frac{1}{2 d} \sum_{e:|e|=1} q_{\beta}(0, z)\left[\xi_{0}(z) \xi_{0}(z+e)-v^{\epsilon}(0, \xi) v^{\epsilon}(e, \xi)\right] .
\end{aligned}
$$

For any bounded function $f$,

$$
\left|E_{v_{\tau}^{\epsilon}}^{\epsilon}\left(f\left(\eta_{t}, \xi_{t}\right)\right)-v_{\tau}^{\epsilon}(f(\eta, \xi))\right| \leqslant \frac{2 t}{\tau} \sup _{s \leqslant \tau+t} \epsilon^{d} \sum_{x \in \Omega_{\epsilon}} E_{\mu^{\epsilon}}^{\epsilon}\left(\left|S_{x} f\left(\eta_{s}, \xi_{s}\right)\right|\right) .
$$

By (E.2), $\left|f^{\epsilon}(\xi)\right| \leqslant 1$, then, for any $T>0$,

$$
E_{\mu^{\epsilon}}^{\epsilon}\left(\sup _{\tau^{\prime} \leqslant \tau}\left|\int_{0}^{\tau^{\prime}} \mathcal{Q}\left(\xi_{t}\right)\right|\right) \leqslant \tau E_{\nu_{\tau}^{\epsilon}}^{\epsilon}\left(\left|f^{\epsilon}\left(\xi_{T}\right)\right|\right)+2 T .
$$

By choosing $T=\mathrm{e}^{M} \epsilon^{2 \beta}$ with $M$ as in (8.2), we get 
1.h.s. of $($ E.3 $) \leqslant \tau E_{v_{\tau}^{\epsilon}}^{\epsilon}\left(\mathbf{1}_{u^{\epsilon}(0, \eta)<M}\left|f^{\epsilon}\left(\xi_{T}\right)\right|\right)+2 \mathrm{e}^{M} \epsilon^{2 \beta}+c(\tau) \epsilon^{(1-\alpha) d}$

and we will then prove (E.1) by showing that

$$
\lim _{\epsilon \rightarrow 0} E_{v_{\tau}^{\epsilon}}^{\epsilon}\left(\mathbf{1}_{u^{\epsilon}(0, \eta)<M} f^{\epsilon}\left(\xi_{T}\right)^{2}\right)=0, \quad T=\mathrm{e}^{M} \epsilon^{2 \beta} .
$$

We write $\underline{x}$ for a subset of $\Omega_{\epsilon},|\underline{x}|$ for its cardinality and call

$$
g_{\underline{x}}(\xi)=\prod_{x \in \underline{x}} \xi(x) .
$$

Then $f^{\epsilon}(\xi)^{2}=\sum_{\underline{x}} c(\underline{x}) g_{\underline{x}}(\xi)(c(\underline{x})$ numerical coefficients) and

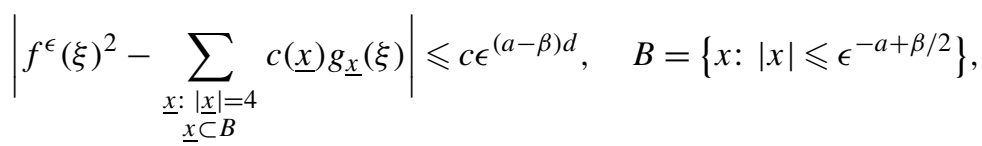

where

$$
\sum_{\underline{x}:|\underline{x}|=4}|c(\underline{x})| \leqslant c, \quad \sum_{\underline{x}:|\underline{x}|<4}|c(\underline{x})| \leqslant c \epsilon^{(a-\beta) d}, \quad \sum_{\underline{x} \not \subset B}|c(\underline{x})| \leqslant c \mathrm{e}^{\epsilon^{-\beta}} .
$$

By (11.4) $E_{v_{\tau}^{\epsilon}}^{\epsilon}\left(\mathbf{1}_{u^{\epsilon}}(0, \eta)<M g_{\underline{x}}\left(\xi_{T}\right)\right)$ is modulo a negligible error a convolution of the form $\pi_{t} * \xi_{0}$ with a suitable $t$. The following computation shows that with a negligible error such a convolution can be rewritten as a new convolution involving $v^{\epsilon}$ for which we have good smoothness properties.

$$
\begin{aligned}
& \sum_{z} \pi_{\epsilon^{-2(a-\beta)} \mathrm{e}^{M-u^{\epsilon}\left(0, \eta_{0}\right)}}(x, z) \xi_{0}(z)=\sum_{y} \pi_{\epsilon^{-2(a-\beta)}\left[\mathrm{e}^{M-u^{\epsilon}\left(0, \eta_{0}\right)}-1\right]}(x, y)\left\{\sum_{z} q_{\beta}(y, z) \xi_{0}(z)\right\} \\
&=\sum_{y} \pi_{\epsilon^{-2(a-\beta)}\left[\mathrm{e}^{M-u^{\epsilon}\left(0, \eta_{0}\right)}-1\right]}(x, y) v^{\epsilon}\left(y, \xi_{0}\right)=v^{\epsilon}\left(0, \xi_{0}\right)+R^{\epsilon}, \\
& R^{\epsilon}=\sum_{y} \pi_{\epsilon^{-2(a-\beta)}\left[\mathrm{e}^{M-u^{\epsilon}\left(0, \eta_{0}\right)}-1\right]}(x, y)\left[v^{\epsilon}\left(y, \xi_{0}\right)-v^{\epsilon}\left(0, \xi_{0}\right)\right] .
\end{aligned}
$$

By Theorem 8.3 and the analogue of Corollary 8.1,

$$
E_{v_{\tau}^{\epsilon}}^{\epsilon}\left(\left|v^{\epsilon}\left(y, \xi_{0}\right)-v^{\epsilon}\left(0, \xi_{0}\right)\right|\right) \leqslant c \epsilon^{a}|y|
$$

so that $\left|R^{\epsilon}\right| \leqslant c^{\prime} \epsilon^{a} \epsilon^{-a+\beta}$. Then

$$
\lim _{\epsilon \rightarrow 0} \sup _{\underline{x} \subset B,|\underline{x}|=4}\left|E_{v_{\tau}^{\epsilon}}^{\epsilon}\left(\mathbf{1}_{u^{\epsilon}}(0, \eta)<M g_{\underline{x}}\left(\xi_{T}\right)\right)-E_{v_{\tau}^{\epsilon}}^{\epsilon}\left(v^{\epsilon}\left(y, \xi_{0}\right)^{4}\right)\right|=0 .
$$

Hence (E.4), using (E.5)-(E.6), thus concluding the proof of (E.1).

\section{Appendix F. Proof of Theorem 6.1. Conclusion}

With reference to (C.19) and (C.21), we call $A$ the $2 \times 2$, matrix with entries $c_{1}, c_{2}, c_{3}, c_{4}$ and we define the following two dimensional vector $\mathbf{R}^{\epsilon}(t)=\left(R_{1}^{\epsilon}(t), R_{2}^{\epsilon}(t)\right)$

$$
R_{i}^{\epsilon}(t):=\int_{0}^{t} \mathfrak{R}_{i}^{\epsilon}\left(\xi_{s}, \eta_{s}\right) \mathrm{d} s+M_{i}(t), \quad i=1,2,
$$

where $M_{1}(t)$ and $M_{2}(t)$ are the mean zero martingales defined in (4.4) and (4.5) respectively. Thus (6.1) is proven. From (C.20) and (C.22) it follows that (6.2) holds for the first term on the right-hand side of (F.1).

We are thus left with the proof that also the martingale terms verify (6.2). We first notice that

$$
\left(\mathbb{E}_{\mu_{\epsilon}}\left(\sup _{s \leqslant t}\left|M_{i}(s)\right|\right)\right)^{2} \leqslant \mathbb{E}_{\mu_{\epsilon}}\left(\sup _{s \leqslant t} M_{i}(s)^{2}\right) \leqslant 4 \mathbb{E}_{\mu_{\epsilon}}^{\epsilon}\left(M_{i}(t)^{2}\right), \quad i=1,2,
$$


and then write (see for instance Chapter 2 of [4]):

$$
M_{i}(t)^{2}-\int_{0}^{t} \gamma_{i}(s) \mathrm{d} s=: N_{i}(t) \text { is a martingale, } i=1,2,
$$

where the compensators $\gamma_{i}$ of $M_{i}^{2}, i=1,2$, are given in (F.3) below,

$$
\gamma_{i}(s)=L X_{i}^{2}-2 X_{i} L X_{i}, \quad i=1,2,
$$

where

$$
X_{1}(s)=\left\|u^{\epsilon}\left(\eta_{s}\right)-U^{\epsilon}(s)\right\|^{2}, \quad X_{2}(s)=\left\|v^{\epsilon}\left(\xi_{s}\right)-V^{\epsilon}(s)\right\|^{2} .
$$

Going back to (F.2), we have for all $t>0$,

$$
\mathbb{E}_{\mu_{\epsilon}}^{\epsilon}\left(M_{i}(t)^{2}\right)=\mathbb{E}_{\mu_{\epsilon}}^{\epsilon} t\left(N_{i}(t)+\int_{0}^{t} \gamma_{i}(s) \mathrm{d} s\right) \leqslant \mathbb{E}_{\mu_{\epsilon}}^{\epsilon}\left(X_{i}(0)^{2}\right)+t \sup _{s \leqslant t} \mathbb{E}_{\mu_{\epsilon}}^{\epsilon}\left(\left|\gamma_{i}(s)\right|\right) .
$$

Since by Theorem 5.1 the first term on the right-hand side of (F.4) vanishes in the limit $\epsilon \rightarrow 0$, the proof of Theorem 6.1 is concluded by the next Lemma.

Lemma F.1. For any $t>0$,

$$
\limsup _{\epsilon \rightarrow 0} \mathbb{E}_{s \leqslant t}^{\epsilon} \mu_{\epsilon}\left(\left|\gamma_{i}(s)\right|\right)=0 \text {. }
$$

Proof. We first compute $\gamma_{1}$ and, recalling that $L^{(\eta)}$ is the sum of three generators, we get for each of them the sum of three terms that we classify as $\mathcal{A}, \mathcal{B}$ and $\mathcal{C}$ remainders.

$$
L^{(\eta)}\|u-U\|^{4}-2\|u-U\|^{2} L^{(\eta)}\left(\|u-U\|^{2}\right)=\sum_{i=1}^{3}\left[\mathcal{A}_{i}+4 \mathcal{B}_{i}+4 \mathcal{C}_{i}\right],
$$

where

$$
\begin{aligned}
& \mathcal{A}_{1}=\epsilon^{-2} \epsilon^{2 d} \sum_{x, y, z} \eta(z)\left|\nabla p_{\alpha}(x, z)\right|^{2}\left|\nabla p_{\alpha}(y, z)\right|^{2}, \\
& \mathcal{B}_{1}=\epsilon^{-2} \epsilon^{2 d} \sum_{x, y, z}[u(y)-U(y)] \eta(z) \sum_{e:|e|=1}\left|e \cdot \nabla p_{\alpha}(x, z)\right|^{2} e \cdot \nabla p_{\alpha}(y, z), \\
& \mathcal{C}_{1}=\epsilon^{-2} \epsilon^{2 d} \sum_{x, y, z}[u(y)-U(y)][u(x)-U(x)] \eta(z) \nabla p_{\alpha}(x, z) \cdot \nabla p_{\alpha}(y, z), \\
& \mathcal{A}_{2}=\kappa \epsilon^{2 d} \sum_{x, y, z} \eta(z) p_{\alpha}(x, z)^{2} p_{\alpha}(y, z)^{2}, \\
& \mathcal{B}_{2}=\kappa \epsilon^{2 d} \sum_{x, y, z}[u(y)-U(y)] \eta(z) p_{\alpha}(x, z)^{2} p_{\alpha}(y, z), \\
& \mathcal{C}_{2}=\kappa \epsilon^{2 d} \sum_{x, y, z}[u(y)-U(y)][u(x)-U(x)] \eta(z) p_{\alpha}(x, z) p_{\alpha}(y, z) .
\end{aligned}
$$

Finally, calling $\varphi(\eta, \xi ; z)=\eta(z) \kappa_{\eta(z)}[1-\xi(z)]+\eta(z) \kappa_{\eta(z)+1} \xi(z)$,

$$
\begin{aligned}
& \mathcal{A}_{3}=\epsilon^{2 d} \sum_{x, y, z} \varphi(\eta, \xi ; z) p_{\alpha}(x, z)^{2} p_{\alpha}(y, z)^{2}, \\
& \mathcal{B}_{3}=-\epsilon^{2 d} \sum_{x, y, z}[u(y)-U(y)] \varphi(\eta, \xi ; z) p_{\alpha}(x, z)^{2} p_{\alpha}(y, z), \\
& \mathcal{C}_{3}=\epsilon^{2 d} \sum_{x, y, z}[u(y)-U(y)][u(x)-U(x)] \varphi(\eta, \xi ; z) p_{\alpha}(x, z) p_{\alpha}(y, z) .
\end{aligned}
$$


By (8.1), the estimates at the end of Section 4 and (8.9) (details are omitted)

$$
\lim _{\epsilon \rightarrow 0} \sum_{i=1}^{3} \sup _{s \leqslant t} \mathbb{E}_{\mu_{\epsilon}}\left(\left|\mathcal{A}_{i}(s)+4 \mathcal{B}_{i}(s)+4 \mathcal{C}_{i}(s)\right|\right)=0 .
$$

We next compute $\gamma_{2}$ and, as before, for each of the three generators we get the sum of three terms that we classify as $\overline{\mathcal{A}}, \overline{\mathcal{B}}$ and $\overline{\mathcal{C}}$ remainders.

$$
L^{(\xi)}\|v-V\|^{4}-2\|v-V\|^{2} L^{(\xi)}\left(\|v-V\|^{2}\right)=\sum_{i=1}^{3}\left[\overline{\mathcal{A}}_{i}+4 \overline{\mathcal{B}_{i}}+4 \overline{\mathcal{C}_{i}}\right],
$$

where, calling $\psi_{e}(\xi, \eta ; z)=\xi(z)[1-\xi(z+e)] \mathbf{1}_{\eta(x+e)=0}$,

$$
\begin{aligned}
& \overline{\mathcal{A}}_{1}=\epsilon^{-2 a} \epsilon^{2 d} \sum_{x, y, z} \sum_{e:|e|=1} \psi_{e}(\xi, \eta ; z)\left|\nabla q_{\beta}(x, z)\right|^{2}\left|\nabla q_{\beta}(y, z)\right|^{2}, \\
& \overline{\mathcal{B}_{1}}=\epsilon^{-2 a} \epsilon^{2 d} \sum_{x, y, z}[v(y)-V(y)] \sum_{e:|e|=1} \psi_{e}(\xi, \eta ; z)\left|e \cdot \nabla q_{\beta}(x, z)\right|^{2} e \cdot \nabla q_{\beta}(y, z), \\
& \overline{\mathcal{C}_{1}}=\epsilon^{-2 a} \epsilon^{2 d} \sum_{x, y, z}[v(y)-V(y)][v(x)-V(x)] \psi_{e}(\xi, \eta ; z) \nabla q_{\beta}(x, z) \cdot \nabla q_{\beta}(y, z), \\
& \overline{\mathcal{A}}_{2}=\frac{\kappa^{\prime}}{2 d} \epsilon^{2 d} \sum_{x, y, z} \psi_{e}(\xi, \eta ; z) q_{\beta}(x, z-e)^{2} q_{\beta}(y, z-e)^{2}, \\
& \overline{\mathcal{B}_{2}}=\frac{\kappa^{\prime}}{2 d} \epsilon^{2 d} \sum_{x, y, z}[v(y)-V(y)] \psi_{e}(\xi, \eta ; z) q_{\beta}(x, z-e)^{2} q_{\beta}(y, z-e), \\
& \overline{\mathcal{C}_{2}}=\frac{\kappa^{\prime}}{2 d} \epsilon^{2 d} \sum_{x, y, z}[v(y)-V(y)][v(x)-V(x)] \psi_{e}(\xi, \eta ; z) q_{\beta}(x, z-e) q_{\beta}(y, z-e) .
\end{aligned}
$$

Finally, calling $\phi(\eta, \xi ; z)=\xi(z) \kappa_{\eta(z)+1}$,

$$
\begin{aligned}
& \overline{\mathcal{A}}_{3}=\epsilon^{2 d} \sum_{x, y, z} \phi(\eta, \xi ; z) q_{\beta}(x, z)^{2} q_{\beta}(y, z)^{2}, \\
& \overline{\mathcal{B}}_{3}=-\epsilon^{2 d} \sum_{x, y, z}[v(y)-V(y)] \phi(\eta, \xi ; z) q_{\beta}(x, z)^{2} q_{\beta}(y, z), \\
& \overline{\mathcal{C}_{3}}=\epsilon^{2 d} \sum_{x, y, z}[v(y)-V(y)][v(x)-V(x)] \phi(\eta, \xi ; z) q_{\beta}(x, z) q_{\beta}(y, z) .
\end{aligned}
$$

Since the variables are bounded by 1, by (8.17) and the estimates at the end of Section 4 (details are again omitted)

$$
\lim _{\epsilon \rightarrow 0} \sum_{i=1}^{3} \sup _{s \leqslant t} \mathbb{E}_{\mu_{\epsilon}}\left(\left|\overline{\mathcal{A}}_{i}(s)+4 \overline{\mathcal{B}}_{i}(s)+4 \overline{\mathcal{C}}_{i}(s)\right|\right)=0 .
$$

Lemma F.1 is proved.

\section{Acknowledgements}

Two of us, A.D.M. and E.P., acknowledge very kind hospitality at the Max Planck Institute. We are indebted to a Referee of AHP for many very helpful comments. The research has been partially supported by MURST, COFIN and NATO Grant PST.CLG.976552. 


\section{References}

[1] C. Boldrighini, A. De Masi, A. Pellegrinotti, Non equilibrium fluctuations in particle systems modelling reaction-diffusion equations, Stochastic Process. Appl. 42 (1992) 1-30.

[2] C. Boldrighini, A. De Masi, A. Pellegrinotti, E. Presutti, Collective phenomena in interacting particle systems, Stochastic Process. Appl. 25 (1987) $137-152$.

[3] T. Brox, H. Rost, Equilibrium fluctuations of stochastic particle systems: the role of conserved quantities, Ann. Probab. 12 (1984) $742-759$.

[4] A. De Masi, E. Presutti, Mathematical Methods for Hydrodynamical Limits, Lecture Notes in Mathematics, vol. 1501, Springer-Verlag, 1991.

[5] A. De Masi, E. Presutti, E. Scacciatelli, The weakly asymmetric simple exclusion process, Ann. Inst. H. Poincaré A 25 (1989) 1-38.

[6] P. Ferrari, E. Presutti, E. Scacciatelli, M.E. Vares, The symmetric simple exclusion process. I. Probability estimates, Stochastic Process. Appl. (1991) 89-105.

[7] P. Ferrari, E. Presutti, E. Scacciatelli, M.E. Vares, The symmetric simple exclusion process. II. Applications, Stochastic Process. Appl. (1991) 107-115.

[8] M.Z. Guo, G.C. Papanicolaou, S.R.S. Varadhan, Non linear diffusion limit for a system with nearest neighbor interactions, Comm. Math. Phys. 118 (1988) 31-59.

[9] C. Kipnis, C. Landim, Scaling Limits of Interacting Particle Systems, Springer, 1999.

[10] S. Luckhaus, L. Triolo, The continuum reaction-diffusion limit of a stochastic cellular growth model, Rend. Acc. Lincei (S.9) 15 (2004) 215-223.

[11] M. Mourragui, Comportement hydrodynamique et entropie relative des processus de sauts, de naissances et de morts, Ann. Inst. H. Poincaré Probab. Statist. 32 (1996) 361-385.

[12] A. Perrut, Hydrodynamic limits for a two-species reaction-diffusion process, Ann. Appl. Probab. 10 (2000) $163-191$. 